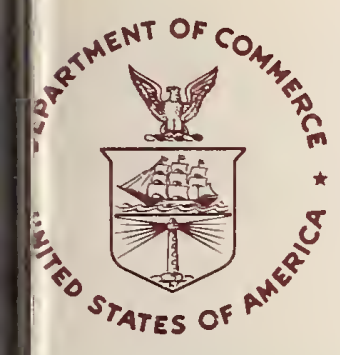

NBS TECHNICAL NOTE 975

U.S. DEPARTMENT OF COMMERCE / National Bureau of Standards

\title{
Results and Analysis of a Round-Robin Test Program for Liquid-Heating Flat-Plate Solar Collectors
}

53

975

18 
The National Bureau of Standards' was established by an act of Congress March 3, 1901. The Bureau's overall goal is to strengthen and advance the Nation's science and technology and facilitate their effective application for public benefit. To this end, the Bureau conducts research and provides: (1) a basis for the Nation's physical measurement system, (2) scientific and technological services for industry and government, (3) a technical basis for equity in trade, and (4) technical services to promote public safety. The Bureau's technical work is performed by the National Measurement Laboratory, the National Engineering Laboratory, and the Institute for Computer Sciences and Technology.

THE NATIONAL MEASUREMENT LABORATORY provides the national system of physical and chemical and materials measurement; coordinates the system with measurement systems of other nations and furnishes essential services leading to accurate and uniform physical and chemical measurement throughout the Nation's scientific community, industry, and commerce; conducts materials research leading to improved methods of measurement, standards, and data on the properties of materials needed by industry, commerce, educational institutions, and Government; provides advisory and research services to other Government Agencies; develops, produces, and distributes Standard Reference Materials; and provides calibration services. The Laboratory consists of the following centers:

Absolute Physical Quantities ${ }^{2}$ - Radiation Research - Thermodynamics and Molecular Science - Analytical Chemistry - Materials Science.

THE NATIONAL ENGINEERING LABORATORY provides technology and technical services to users in the public and private sectors to address national needs and to solve national problems in the public interest; conducts research in engineering and applied science in support of objectives in these efforts; builds and maintains competence in the necessary disciplines required to carry out this research and technical service; develops engineering data and measurement capabilities; provides engineering measurement traceability services; develops test methods and proposes engineering standards and code changes; develops and proposes new engineering practices; and develops and improves mechanisms to transfer results of its research to the utlimate user. The Laboratory consists of the following centers:

Applied Mathematics - Electronics and Electrical Engineering ${ }^{2}$ - Mechanical Engineering and Process Technology ${ }^{2}$ - Building Technology - Fire Research Consumer Product Technology - Field Methods.

THE INSTITUTE FOR COMPUTER SCIENCES AND TECHNOLOGY conducts research and provides scientific and technical services to aid Federal Agencies in the selection, acquisition, application, and use of computer technology to improve effectiveness and economy in Government operations in accordance with Public Law 89-306 (40 U.S.C. 759), relevant Executive Orders, and other directives; carries out this mission by managing the Federal Information Processing Standards Program, developing Federal ADP standards guidelines, and managing Federal participation in ADP voluntary standardization activities; provides scientific and technological advisory services and assistance to Federal Agencies; and provides the technical foundation for computer-related policies of the Federal Government. The Institute consists of the following divisions:

Systems and Software - Computer Systems Engineering - Information Technology.

'Headquarters and Laboratories at Gaithersburg, Maryland, unless otherwise noted; mailing address Washington,D.C. 20234.

Some divisions within the center are located at Boulder, Colorado, 80303. 


\section{Results and Analysis of a Round-Robin Test Program for Liquid-Heating Flat-Plate Solar Collectors}

E. R. Streed ${ }^{1}$, W. C. Thomas ${ }^{2}$, A. G. Dawson, III ${ }^{2}$,

B. D. Wood ${ }^{3}$, and J. E. Hill ${ }^{1}$

${ }^{1}$ Center for Building Technology

National Engineering Laboratory

National Bureau of Standards

Washington, DC 20234

${ }^{2}$ Department of Mechanical Engineering

Virginia Polytechnic Institute and

State University

Blacksburg, VA 24060

${ }^{3}$ Department of Mechanical Engineering

Arizona State University

Tempe, Arizona 85281

Sponsored by the

Department of Energy

20 Massachusetts Avenue, NW

Washington, DC 20545

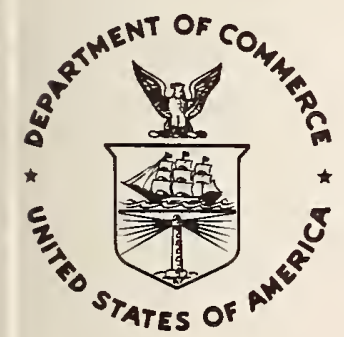

U.S. DEPARTMENT OF COMMERCE, Juanita M. Kreps, Secretary

Dr. Sidney Harman, Under Secretary

Jordan J. Baruch, Assistant Secretary for Science and Technology

NATIONAL BUREAU OF STANDARDS, Ernest Ambler, Director

Issued August 1978 


\section{National Bureau of Standards Technical Note 975}

Nat. Bur. Stand. (U.S.), Tech. Note 975,119 pages (Aug. 1978)

CODEN: NBTNAE

\section{U.S. GOVERNMENT PRINTING OFFICE WASHINGTON: 1978}

For sale by the Superintendent of Documents, U.S. Government Printing Office, Washington, D.C. 20402 Price \$3 Stock No. 003-003-01959-3

(Add 25 percent additional for other than U.S. mailing). 
In discussing this round robin testing program, certain commercial components were used and are identified in order to provide a descriptive characterization of their features. Inclusion of a given component in this report in no case implies a recommendation or endorsement by the National Bureau of Standards, and the presentation should not be construed as a certification that any component would provide the indicated performance. Similarly, the omission of a component does not imply that its capabilities are less than those of the included components. This report is intended to be informative and instructive and not an evaluation of any commercially available components. 
Abstract

1. Introduction

2. Collector Performance

3. Collector Test Procedure

4. Round Robin Program Description

5. Results and Statistical Analysis of Data

6. Analysis of the Effect of Environmental Conditions

7. Analysis of the Effect of Random and Systematic Errors

8. Implication of Collector Performance Uncertainty on System Performance

9. Conclusions

10. References

Appendix A - List of Round Robin Participants

Appendix B - Data Used in Analyzing the Effect of Environmental Conditions

Appendix C-Analytical Relationships Used in the Collector Model for Analyzing the Effect of Environmental Conditions 


\section{Results and Analysis of a Round Robin \\ Test Program for Liquid-Heating \\ Flat-Plate Solar Collectors}

\section{By}

Elmer R. Streed, William C. Thomas, Aaron G. Dawson, III Byard D. Wood, and James E. Hill

\section{Abstract}

A round robin test program was conducted at 21 United States test facilities, using a common test procedure, to determine the intercomparability of thermal performance data pertaining to two liquid-heating flat-plate solar collectors.

The statistical analysis of the data revealed a relatively large spread in the measured values of collector efficiency. Data from approximately half the facilities were then selected for detailed analysis. A collector analytical model was used to show that less than one-third of the mean-square distance could be attributed to different environmental conditions from facility to facility. It was found that the data showed less scatter for one of the two collectors than for the other. In general, the data were consistent for any single facility; most of the scatter was therefore attributed to systematic uncertainties from facility to facility. When the data from six participants reportedly adhering to the requirements of ASHRAE Standard 93-77 were analyzed, the scatter was found to be within normal limits expected for the test procedure.

Key Words: Measurement; modelling; solar; standards; testing 

A proposed procedure for testing and rating solar collectors based on thermal performance was first published by the National Bureau of Standards (NBS) in 1974 [1-3]. The procedure prescribed that a series of outdoor steadystate tests be conducted to determine the near-normal-incidence efficiency of the collector over a range of collector operating temperature conditions. The American Society of Heating, Refrigerating and Air-Conditioning Engineers (ASHRAE) has recently adopted ASHRAE Standard 93-77 [4], which is similar to the original NBS procedure but calls for additional tests to determine the collector time constant, as well as an incident angle correction factor that can be applied to the near-normal-incidence efficiency to determine collector performance as a function of incidence angle.

Since the publication of the NBS procedure [1], several testing loops have been built at the NBS site in Gaithersburg, Md. and experiments conducted to verify the applicability of the test procedure to a variety of commerciallyavailable collectors. In addition, experiments have been conducted to support the development of the new tests which have been included as part of ASHRAE Standard 93-77. The results of these experiments will be forthcoming in a separate publication.

A second major part of the NBS solar collector test development work has consisted of the round robin testing program described in this report. The purpose of the program was to have a variety of testing laboratories across the United States attempt to utilize the test procedure and then determine the extent to which the results differed or were comparable.

\section{Collector Performance}

The performance of flat-plate collectors operating under steady-state conditions can be described by the following relationship [5]:

$$
\frac{q_{u}}{A}=I(\tau \alpha)_{e}-U_{L}\left(\bar{t}_{p}-t_{a}\right)
$$

$$
\begin{aligned}
& \text { where } \mathrm{q}_{\mathrm{u}}=\text { useful heat output, } \mathrm{W} \\
& \begin{aligned}
\mathrm{A} \quad & \text { cross-sectional area, } \mathrm{m}^{2} \\
\mathrm{I} & \text { total solar energy incident upon the plane of the } \\
& \text { collector per unit time per unit area, } \mathrm{W} / \mathrm{m}^{2}
\end{aligned} \\
& (\tau \alpha)_{\mathrm{e}}=\begin{array}{l}
\text { effective transmittance-absorptance product for } \\
\text { the cover plate-absorber combination }
\end{array}
\end{aligned}
$$




$$
\begin{aligned}
& \begin{aligned}
\mathrm{U}_{\mathrm{L}}= & \text { heat transfer } 1 \text { oss coefficient for the solar } \\
& \text { collector, } \mathrm{W} /\left(\mathrm{m}^{2} \cdot{ }^{\circ} \mathrm{C}\right)
\end{aligned} \\
& \begin{aligned}
\bar{t}_{p}= & \text { average temperature of the absorber surface of } \\
& \text { the solar collector, }{ }^{\circ} \mathrm{C}
\end{aligned} \\
& \mathrm{t}_{\mathrm{a}}=\text { ambient air temperature, }{ }^{\circ} \mathrm{C}
\end{aligned}
$$

If one introduces the definition of either the collector efficiency factor $\mathrm{F}^{\prime}$ or the collector heat removal factor $\mathrm{F}_{\mathrm{R}}[6]$, equation (1) can be rewritten*

$$
\frac{q_{u}}{A}=F^{\prime} I(\tau \alpha) e-F^{\prime} U_{L} \frac{t_{f, i}+t_{f, e}}{2}-t_{a}
$$

or

$$
\frac{q_{u}}{A}=F_{R} I(\tau \alpha)_{e}-F_{R} U_{L}\left(t_{f, i}-t_{a}\right)
$$

where

$$
\begin{aligned}
t_{f, e}= & \text { temperature of the heat transfer fluid } \\
& \text { leaving the collector, }{ }^{\circ} \mathrm{C} \\
t_{f, i}= & \text { temperature of the heat transfer fluid } \\
& \text { entering the collector, }{ }^{\circ} \mathrm{C}
\end{aligned}
$$

If the solar collector efficiency is defined by

$$
n=\frac{q_{u}}{A I}
$$

then the efficiency can be written as

$$
n=(\tau \alpha)_{e}-U_{L} \frac{\bar{t}_{p}-t_{a}}{I}
$$

\footnotetext{
* Equation (2) is only approximately correct. The collector efficiency factor $\mathrm{F}^{\prime}$ is defined in terms of the local fluid temperature and since the fluid temperature varies exponentially from inlet to exit, equation (2) is incorrect. The error is insignificant for flat-plate collectors using a liquid heat transfer fluid but can be significant for those using air.
} 


$$
n=F^{\prime}(\tau \alpha)_{e}-F^{\prime} U_{L} \frac{\frac{t_{f, i}+t_{f, e}}{2}-t_{a}}{I}
$$

or

$$
\eta=F_{R}(\tau \alpha)_{e}-F_{R} U_{L} \frac{t_{f, i}-t_{a}}{I}
$$

Regardless of which form of the efficiency equation is used, equations (5-7) indicate that if the efficiency is plotted against some appropriate parameter $(\Delta t / I)$, a straight line will result where the slope is some function of $\mathrm{U}_{\mathrm{L}}$ and the $\mathrm{y}$ intercept is some function of $(\tau \alpha)_{\mathrm{e}}$. In reality $\mathrm{U}_{\mathrm{L}}$ is not a constant but rather a function of the operating temperature of the collector and of the ambient weather conditions such as air temperature, sky temperature, and wind velocity and direction. In addition, $(\tau \alpha)_{e}$ varies with incident angle to the collector and can vary to some extent as a function of the spectral and spatial distribution of the incoming solar radiation.

\section{Collector Test Procedure}

The procedure proposed by NBS in references 1 and 2 is based on collectors that can be isolated so that they have effectively one inlet and one outlet. The energy of the fluid entering and leaving the collector can be determined by making appropriate measurements. These quantities are then compared to the energy incident upon the collector (also determined by measurement) in order to calculate the collector efficiency. The fluid can be either a liquid or a gas but not a combination of the two.

As part of the procedure, the apparatus to be used is specified when the heat transfer fluid is a liquid (Figure 1) or air (Figure 2). The detailed requirements of the apparatus are given along with specifications for instrumentation to be used in measuring incident solar radiation, temperature, temperature difference, liquid flow rate, air flow rate, pressure, pressure drop, time and weight. For the specification of instrumentation, emphasis was placed on utilizing existing standards and other manuals of acceptable practice.

The series of tests consists of determining the average efficiency for 15-minute periods (integrating the energy quantities) over a range of temperature differences between the average fluid temperature and the ambient air. The efficiency is then calculated by

$$
\eta=\frac{\int_{0}^{\tau} \dot{m}_{p}\left(t_{f, e}-t_{f, i}\right) d \tau}{A \int_{0}^{\tau} I d \tau}
$$




$$
\begin{aligned}
& \dot{\mathrm{m}}=\text { mass flow rate of the heat transfer fluid, } \mathrm{kg} / \mathrm{s} \\
& c_{p}=\text { specific heat of the heat transfer fluid, } \mathrm{J} /\left(\mathrm{kg} \cdot{ }^{\circ} \mathrm{C}\right)
\end{aligned}
$$

The flow rate is required to be steady and vary by less than \pm 1.0 percent for the duration of each test. In addition, the heat transfer fluid shall have a known specific heat which varies by less than 0.5 percent over the temperature range of the fluid during a particular 15-minute test period. Consequently, the efficiency can be determined by

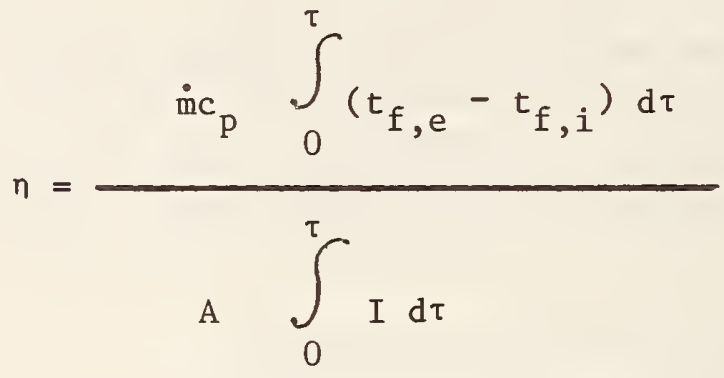

The test apparatuses specified in references 1 and 2 have been designed so that the temperature of the fluid entering the collector can be controlled to selected values. This feature is used to obtain the data over the temperature range desired. At least 16 data points are required for a complete test series and they must be symmetrical with respect to solar noon (to prevent biased results due to possible transient effects).

During each test period, the incident solar radiation must be "quasisteady" as indicated in Figure 3 (in contrast to days in which cloud cover can cause a time distribution such as shown in Figure 4). Other requirements that must be satisfied for each data point are that the 15-minute average insolation be greater than $630 \mathrm{~W} / \mathrm{m}^{2}$ and the incident angle between the direct solar beam and the outward drawn normal from the collector be less than $45^{\circ}$. In addition, the range of ambient temperatures for the entire test series must be less than $30^{\circ} \mathrm{C}$.

In developing the test procedure and writing the specific requirements, one main area of concern was ensuring that the measurements made would be sufficiently accurate so that the collector efficiency values would be meaningful.

It is recommended that the temperature rise in the heat transfer fluid passing through the solar collector be measured with either a thermopile (air or liquid as the transfer fluid) or calibrated resistance thermometers (only with a liquid). It is felt that an accuracy of $\pm 0.1^{\circ} \mathrm{C}$ is possible with such sensors and associated read-out devices. A variety of liquid flow meters are available that will enable $\dot{m}$ to be determined to within \pm 1 percent of the measured value. The measurement of the incident solar radiation using pyranometers is perhaps the most critical and least accurate of all the measurements specified. As described in reference 2 , typical accuracies of better 
than 5 percent are difficult to attain. However, Latimer has shown [7] that it is possible to obtain an accuracy of \pm 2.3 percent with the better pyranometers provided they have been calibrated directly against a primary or a working standard pyrheliometer using the sun as a source and that they are properly installed and maintained. Based on this analysis, the test procedure should result in efficiency values accurate to within $\pm 4-5$ percent.

As mentioned previously, ASHRAE has recently adopted Standard 93-77 which is similar to the NBS procedure. It was not adopted in time to be specified in this round robin test program; however, its main features will be described here for purposes of comparison.

The major changes in the conduct of the efficiency tests as specified in Standard 93-77 compared to that specified in the NBS procedures [1 and 2] are as follows:

1. The testing apparatus for water-cooled collectors has been modified to include a storage tank for damping out thermal transients and a by-pass for periodically calibrating the flow meter in place.

2. The testing apparatus for air heaters has been rearranged so that air is "pulled" through the collector instead of being blown through it (slight negative gage pressure in the collector).

3. More stringent requirements have been included for the measurement of solar radiation. Only pyranometers which meet or exceed the characteristics of a first class pyranometer as classified by the World Meteorological Organization [8] are allowed.

4. In conducting the test, data must be taken when the incident angle is less than $30^{\circ}$ (compared to $45^{\circ}$ in $[1,2]$ ).

5. The time period required for the integration of energy quantities to compute one efficiency value has been decreased from 15 minutes to either 5 minutes or one time constant, whichever is larger.

6. In computing efficiency, the gross frontal area of the collector is used instead of aperture area.

7. The efficiency curve is drawn by plotting efficiency versus the difference between inlet fluid temperature and ambient temperature divided by the incident solar radiation. (Average fluid temperature is used in the NBS procedures [1,2].) Inlet fluid temperature was chosen to be used in the plot primarily because the characteristics of the collector required for the system design procedures as reported in references 9 and 10 can be determined directly from the curve. 
The major new features of Standard 93-77 compared to the NBS procedures $[1,2]$ are:

1. The collector is required to undergo a preconditioning test prior to the start of the thermal tests. The collector must be exposed for three cumulative days with no fluid passing through it and with the mean incident solar radiation measurged in the plane of the collector exceeding 17,000 $\mathrm{kJ} /\left(\mathrm{m}^{2} \cdot \mathrm{day}\right)$.

2. Prior to conducting the efficiency tests, a time constant test is done.

3. After completing the efficiency tests, a series of tests is conducted to determine the collector's incident angle modifier.

4. The entire group of tests may be performed indoors using a solar simulator if desired. The specifications for the simulator are included and follow closely those of references $[11-13]$.

\section{Round Robin Program Description}

Participants in the program were obtained by soliciting interest with a Sources Sought Announcement in the May 15, 1975 Commerce Business Daily and by individual letters to organizations known to be interested in solar collector testing. The program was designed to provide experimental data from various climatic regions and test facilities on two flat-plate liquidheating collectors with different heat transfer and optical properties. Final selection of the organizations was made on the basis of test facility experience, climatic and geographic regions, cost to modify facility and labor to conduct the test, and willingness to conduct the test in accordance with the NBS proposed procedure. A total of 21 organizations, including three Government laboratories, as listed in Appendix A, participated in testing each collector. The distribution of participants with respect to geographical and climatic regions is shown in Figure 5.

Because of the large interest in the program, the relatively large number of climatic regions, and the time required to perform the test, it was impractical to send the same two collectors to each participant. Therefore, collectors were selected with sufficiently stable component properties and from manufacturers with established quality assurance methods capable of keeping thermal property variations to a minimum. One collector of each type was shipped to each participant for testing.

Collector No. 1 (PPG Industries) consisted of two $3.2 \mathrm{~mm}$-thick tempered glass cover-plates and an aluminum absorber plate with a flat-black coating assembled into a sealed unit. The assembly was attached to a sheetmetal box containing glass-fiber insulation as shown in Figure 6. Measurements made 
at the manufacturer's plant of the absorber coating thickness on each of the 21 panels indicated variations from the center to the edge of about $0.0076 \mathrm{~mm}$ in the range of 0.0127 to $0.0203 \mathrm{~mm}$. The relationships between coating solar absorptance $\left(\alpha_{s}\right)$, normal emittance $\left(\varepsilon_{\eta}\right)$ and thickness are shown in Figure 7. Independent measurement of $\alpha_{s}$ and $\varepsilon_{H}$ (hemispherical emittance) using actual coated samples resulted in values of 0.94 and 0.92 , respectively.* The solar transmittance of the glass used in the cover-plate assembly was determined to be 0.85 for an air mass 2 spectral distribution (measurements were made on untempered glass). The glass normal emittance was measured to be 0.85 . While the collectors were carefully selected at the manufacturer's plant, no other provisions were made to control the reproducibility of the sample of collectors used in the program. Analysis of the known variations in collector properties indicates that the efficiences should be reproducible to within 1 or $2 \%$ if measured under identical conditions.

Collector No. 2 consisted of one $3.2 \mathrm{~mm}$-thick tempered glass cover-plate (Chamberlain Manufacturing Company (CMC)) with a seam-welded mild steel absorber coated with a black chrome selective coating. The absorber was mounted on a thermal insulator and backed with glass-fiber insulation. The entire assembly was mounted in a steel frame with a sheetmetal back and aluminum glazing frame as shown in Figure 8. Spectral reflectance measurements made of six $30.48 \mathrm{~cm}^{2}$ specimens coated at the same time as the absorber panels indicated a solar absorptance of the black chrome coating of 0.95 . The normal emittance of the coatings was measured to be $0.14^{* *}$ The coating manufacturer*** also measured the solar absorptance and emittance of each panel using portable optical instrumentation. Those results are included in Table 1.

A list of the pertinent dimensions, optical properties, and materials for each collector is presented in Table 1 .

\section{Results and Statistical Analysis of Data}

The primary result reported by each participant was a plot of collector efficiency, $\eta$, versus $\frac{t_{f, e}+t_{f, i}}{2} / I$ along with the testing conditions for each of the data points. A summary of the data is given in Table 2 and Table 3 for collectors No. 1 and No. 2, respectively. The values of $F^{\prime}(\tau \alpha)$ e and $F^{\prime} U_{L}$ were determined by a first-order least-square-fit to all measured data points. Mean and standard deviation values were calculated for these two parameters for the group, using the following statistical equations:

* Lockheed Palo Alto Research Laboratory, Palo Alto, California.

** Gier-Dunkle Instrument Co., Emissometer Model DB-100, Santa Monica, California

*** 01ympic Plating Company, Canton, Ohio. 


$$
\text { mean }=\bar{x}=\frac{\sum_{i=1}^{n} x_{i}}{n}
$$

and

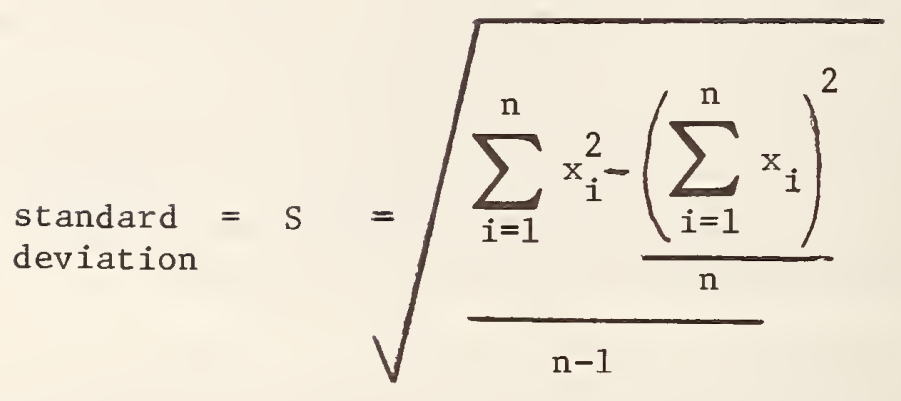

The percent deviation from the mean for each set of data points is also shown in the Tables.

An indication of the spread and distribution of $F^{\prime}(\tau \alpha)$ values for each collector are shown in Figure 9. The greater frequency of values about the mean for collector No. 2 is probably due to the fact that more of the data were taken at smaller incidence angles for this collector than for collector No. 1. An early version of ASHRAE Standard 93-77 was out for public review before most participants started testing collector No. 2. Since it required the incident angle to be less than $30^{\circ}$ (compared to $45^{\circ}$ in the [1, 2]), many participants were undoubtedly influenced to test closer to solar noon.

The relatively large standard deviation of $7.7 \%$ for $F^{\prime}(\tau \alpha)$ for collector No. 1 was not expected. It is felt that one major source of the deviation was the use of a "black-and-white" pyranometer, model 8-48, by eight participants. It has been reported [14] that the calibration factor (determined originally with the instrument in the horizontal position) of a similarlydesigned instrument changed from 3 to $8 \%$ as the instrument was tilted up from the horizontal to typical angles used for collector testing. The mean value of the $F^{\prime}(\tau \alpha)$ e values for those collectors (No. 1) where the "black and white" pyranometer was used is 0.79 compared to a mean value of 0.725 for those collectors where an "all-black" (model PSP) pyranometer was used. By arbitrarily reducing the measured $F^{\prime}(\tau \alpha)$ e values in Group 1 by 0.065 , a new mean value for $\mathrm{F}^{\prime}(\tau \alpha)$ for all 21 participants, of 0.73 would result for collector No. 1. This correction would reduce the standard deviation in $F^{\prime}(\tau \alpha)$ e to \pm 0.05 or $\pm 6.8 \%$.

Only five of the participants used the "black-and-white" pyranometer for collector No. 2 and the standard deviation was only \pm 0.039 or $\pm 4.64 \%$ about the mean of 0.84 . In addition, two participants apparently used the gross area rather than the aperture area to calculate the efficiency, which resulted in a $5 \%$ low value of $F^{\prime}(\tau \alpha)$. Correction of these values would reduce the standard deviation to \pm 0.03 or $\pm 3.6 \%$. 
A similar plot of the distribution of reported values for $\mathrm{F}^{\prime} \mathrm{U}_{\mathrm{L}}$ for both collectors is shown in Figure 10. As can be seen, the variation in reported results is large. The standard deviation about the mean of $6.43 \mathrm{~W} /\left(\mathrm{m}^{2}\right.$. $\left.\mathrm{C}\right)$ for the collector No. 1 is $\pm 1.01 \mathrm{~W} /\left(\mathrm{m}^{2} . q\right)$ or $\pm 15.7 \%$. For collector No. 2, the mean is $4.37 \mathrm{~W} /\left(\mathrm{m}^{2}, \mathrm{C}\right)$ with a standard deviation of $\pm 1.20 \mathrm{~W} /\left(\mathrm{m}^{2} \cdot{ }^{\circ} \mathrm{C}\right)$ or $\pm 27.7 \%$. In order to explain the variation, a close examination was made of the reported data.

As shown in Figure 6 for collector No. 1, the absorber plate is in thermal contact with the outside mounting and cover-plate frame. Therefore, the edge losses are dependent upon the collector array mounting configuration and can be particularly significant when an individual panel is exposed. The influence of edge losses in $F^{\prime} U_{L}$ is shown in Figure 11. The variation, from no edge loss to an edge loss of about 3 times the value of $0.5 \mathrm{~W} /\left(\mathrm{m}^{2}\right.$. $\left.\mathrm{C}\right)$ for a typically insulated edge, results in a percentage difference of $+7 \%$ to $-26 \%$ from the mean value of $\mathrm{F}^{\prime} \mathrm{U}_{\mathrm{L}}$ reported for the collector.

Each participant was requested to enclose the No. 1 collector in a $5 \mathrm{~cm} \mathrm{x}$ $10 \mathrm{~cm}$ frame with a plywood backing to provide a more uniform test situation for this collector. A review of the collector mounting procedures plus the description of the actual tests indicated that some unusual conditions did exist. For example, extra insulation was used around the back and edges of some of the collectors, mass flow rates different from the prescribed $0.02 \mathrm{~kg} /\left(\mathrm{s} \cdot \mathrm{m}^{2}\right)$ were used, and incidence angles exceeded 45 degreesin some cases. By omitting these data from the analysis, a new mean value of $\mathrm{F}^{\prime} \mathrm{U}_{\mathrm{L}}$ of $6.56 \mathrm{~W} /\left(\mathrm{m}^{2}{ }^{\circ} \mathrm{C}\right)$ would result with a standard deviation of $\pm 0.75 \mathrm{~W} /\left(\mathrm{m}^{2} . \stackrel{\circ}{\mathrm{C}}\right)$ or $\pm 11.4 \%$.

The influence of one extreme value for $F^{\prime} U_{L}$ for collector No. 2 had a large effect on the statistical analysis. By considering the value of 1.6 $\mathrm{W} /\left(\mathrm{m}^{2} \cdot{ }^{\circ} \mathrm{C}\right)$ to be an outlyer [15] and therefore omitted, the mean value of $\mathrm{F}^{\prime} \mathrm{U}_{\mathrm{L}}$ would become $4.58 \mathrm{~W} /\left(\mathrm{m}^{2} \cdot{ }^{\circ} \mathrm{C}\right)$ and the standard deviation $\pm 0.775 \mathrm{~W} /\left(\mathrm{m}^{2} \cdot{ }^{\circ} \mathrm{C}\right)$ or $\pm 16.8 \%$.

The significantly lower overall loss coefficient for collector No. 2 is due in part to better thermal insulation of the absorber plate from the exterior environment and the use of a selective coating. However, the single glazing makes the collector heat loss more susceptible to wind and ambient temperature. The range in measured values of overall loss coefficient for the five collectors that exhibited $F^{\prime}(\tau \alpha)$ e values of 0.85 (very close to the corrected mean of 0.844 ) are $+20 \%$ and $-17 \%$ from the mean as $i 11$ strated in Figure 12.

\section{Analysis of the Effect of Environmental Conditions}

It was felt that the range of environmental conditions existing during the tests at the various facilities across the country undoubtedly caused a portion of the data scatter. In order to determine the amount, one of the round robin participants, W.C. Thomas and his graduate student A.G. Dawson, III of Virginia Polytechnic Institute and State University undertook a detailed analysis of the data. The results of that study are reported 
in this Section [16]. The general approach used was to reference all measured thermal efficiencies to a common set of environmental conditions by using analytical models for the two flat-plate collectors tested.

Screening and Selection of Data

The data were examined for complete documentation and consistency with respect to test conditions. The objective was to assemble at least ten complete sets of data. It should be noted that regional location, altitude, and climatic conditions at the testing laboratories were not criteria for screening the data. After the screening process, 12 sets were selected for collector No. 1 (PPG) and 10 sets were selected for collector No. 2 (CMC). Typically, test results were not included in the analysis because diffuse fractions, wind speed, ambient temperature, incident angles, or type of test fluid were not available. In some cases, the calculated results required by [1, 2] were incomplete. Where possible, these were completed and the results used. For example, incident angles were calculated provided the times of testing and collector orientation were reported. Results for more than the required 16 data points were given by some participants. For these cases, the 16 points nearest solar noon were selected. The additional points were not included to avoid weighting the particular facility more heavily than the others.

The data corresponding to the figures to be shown in this Section are tabulated in Appendix B. Testing organizations are identified by the letter designation of Tables 2 and 3 . The solid curves shown are least-squares second-order polynomials unless noted otherwise. Following reference [17], the mean value of the square of the distance from, the points to the curve ("mean square") is used to compare the closeness of correlations.

Figure 13 shows the efficiencies as reported by the 12 selected organizations for collector No. 1. The abscissa values were recalculated from the data so that the efficiency curve could be drawn using inlet fluid temperature instead of average fluid temperature. This abscissa selection makes the plot consistent with [4]. The points shown to the left of the ordinate axis are for tests where the inlet temperature was less than the ambient temperature. Participant $\mathrm{K}$ reported two measurements where the abscissa was outside the range shown. While the results were included in the statistical analysis, the points are not shown in the graphs. The second-order curve shows an increasing slope as $\frac{t_{f, i}-t_{a}}{I}$ increases contrary to what would be expected if all data were taken at one laboratory.

Identification of the data points in Figure 13 with each participant showed, generally, a small amount of scatter for individual organizations. The spread, consequently, results from the differences in efficiency values, at the same conditions, by the various organizations. The ordinate intercept for the second-order curve shown is 0.731 .

Figure 14 shows the results reported for collector No. 2 as reported by the 10 selected participants. Generally, the efficiencies reported by the different laboratories agree more closely than do those in Figure 13. Since 
this was the second collector tested and since the collector was less sensitive to the method of mounting on the test frame, the trend is as expected. The second-order curve has a continuously decreasing slope with an ordinate intercept of 0.80 .

Figures 15 and 16 show a subset of the above-selected test results where each facility shown reportedly adhered to the more stringent requirements of ASHRAE Standard 93-77 [4]. There were five organizations (A, G, H, J, N) testing the collector No. 1 and seven organizations ( $G, H, J, N, 0, R, U$ ) testing collector No. 2 that reportedly met these requirements. Figures 15 and 16 show clearly that the differences are much more pronounced from test facility to test facility compared to the scatter reported by a given facility. There is much less scatter associated with test results shown in Figure 15 and 16 than in Figures 13 and 14, respectively.

\section{Analysis Approach}

The principal task was to determine how much of the scatter about the curves, as shown in Figures 13 through 16, could be attributed to the differences in environmental conditions under which the tests were conducted. The analysis approach involved adjusting each of the efficiency values or data points up or down depending on whether the combined effect of the actual test environment would theoretically result in a higher or lower efficiency if the same test were conducted at a "reference" set of conditions. The criterion for evaluating the effect of the data adjustment was to compare the before and after values of the mean square of the regression analysis.

Measured efficiencies as reported were adjusted to a common set of conditions by subtracting the theoretically-determined efficiency at actual (experimental) conditions from the theoretical efficiency at the common ("reference") condition and adding the result algebraically to the experimentally-determined efficiency. The expression for the corrected efficiency is

$$
\begin{aligned}
n(\text { std. conditions, corrected })= & n \text { (actual conditions, measured) }+ \\
& {[\eta \quad \text { (std. conditions, theoretical) }-} \\
& n \text { (actual conditions, theoretical) }]
\end{aligned}
$$

Applying the correction required a theoretical thermal performance analytical model and the configuration, dimensions, and heat transfer properties for each collector. It should be noted, however, that the theory was used only to determine an efficiency difference. Therefore small uncertainties in collector properties and the analytical model would have had an even smaller effect on the corrected efficiency values.

"Reference" conditions selected were generally the mean values of the reported test conditions reported with round-off to convenient values. This criterion for a set of "reference" conditions resulted in the minimum overall adjustment of data. The "reference" environmental conditions selected are shown in Table $\mathrm{Cl}$ of Appendix C. 
The collector model used was based on state-of-the-art collector theory as summarized in reference [6]. Extensions have been made to account for the effects of the scattered component of solar radiation and temperature and composition of the heat transfer fluid. The presence of scattered radiation effectively changes the transmittance of the cover-plate assembly. Scattered radiation is accounted for by considering two limiting cases. The scattered radiation is assumed to be either completely diffuse or from an apparent origin near the sun.

All the test facilities which furnished the results under consideration used either water or ethylene glycol-water mixtures as the transfer fluid. The properties of glycol mixtures, as functions of temperature and concentration, were taken from reference [18] and programmed as an interpolation table.

The complete details of the model and equations and how they were solved are given in Appendix $\mathrm{C}$.

Use of the Analytical Model

After the data were compiled, a set of cards was prepared for each test facility with operating conditions, environmental conditions, and measured efficiencies encoded. The collector design parameters and "reference" conditions were, of course, the same for all test facilities. Corrections to the measured efficiencies were then made progressively by accounting for one operating environmental variable at a time. Using the inlet fluid temperature in the abscissa rather than the mean fluid temperature resulted in a considerable simplification. Had the mean fluid temperature been used, it would have been necessary to carry out a separate calculation to correct the mean fluid temperature to "reference" conditions. As the corrections to "reference" conditions were applied progressively, the mean squares were used to evaluate the effectiveness of that particular correction. In addition, the graphs were studied visually by superimposing them on previous graphs to observe trends.

A separate statistical analysis plot routine and package were used to prepare the graphs. The graphs were drawn by a Calcomp plotter in conjunction with Virginia Polytechnic Institute and State University's IBM 370/158 digital computer. Second-order polynomial representations of collector efficiency were used since the representation essentially duplicated the results of the analytical model at "reference" conditions. (The mean squares of the fits to the standard theoretical curves were less than 0.02). Other considerations that were taken into account in the decision to use the second-order equation include the requirements of ASHRAE 93-77 [4] and the observation that a linear fit gives somewhat higher than expected values of the ordinate intercept $\mathrm{F}_{\mathrm{R}}(\tau \alpha) \mathrm{e}$

$\underline{\text { Results }}$

The results with all the environmental and operating conditions considered are shown graphically in this section and in tabular form in Appendix B. 
In all the graphs that show discrete efficiency points, the solid lines are least-squares curves rather than a comparison per se with the theoretical model.

Figures 17 through 20 show the theoretical effect on efficiency of environmental conditions. The abscissas correspond approximately to the ranges reported in the round robin program. The curves show the effect of a single parameter with the others constant at "reference" values. Note that $\left(t_{f, i}-t_{a}\right) / I$ is held constant for each plot. The effects of parameters depend strongly on the inlet temperature. The two inlet temperatures selected to depict the results are $10^{\circ} \mathrm{C}$ and $70^{\circ} \mathrm{C}$ above ambient which brackets the recommended test range [1, 2, 4]. In view of Figures 17 through 20, the expected efficiency changes resulting from correcting to "reference" conditions are generally not large, as will be shown below, compared to the more extreme differences noted in Figures 13 through 16.

Figure 21 shows the theoretical efficiency values calculated for collector No. 1 for the actual conditions reported by the 12 selected participants. The curve has a slightly increasing slope. The shape indicates that this combination of environmental conditions overshadows the increase of $\mathrm{U}_{\mathrm{L}}$ with temperature. The scatter about the curve is, of course, justified in the absence of any experimental error. The efficiency spread at the abscissa value of $0.07\left({ }^{\circ} \mathrm{C} \cdot \mathrm{m}^{2}\right) / \mathrm{W}$ is pronounced but does not completely account for the spread at the corresponding value in Figures 13 and 15 . Figure 22 shows the calculated efficiency for collector No. 1 at "reference" conditions. Figure 23 shows efficiencies corrected for all environmental and operating conditions. The mean squares are reduced from 27.9 in Figure 13 to 20.6 (percentage points-squared) in Figure 23. It is significant to note that the increasing slope is essentially removed in Figure 23 as a result of the correction.

Figure 24 shows the theoretical efficiencies calculated for the collector No. 1 tests that reportedly met ASHRAE 93-77 requirements. The slightly increasing slope observed in Figure 15 is suppressed and the scatter is not as large. Figure 25 shows the corrected efficiencies. The mean square is reduced from 23.2 in Figure 15 to 17.1 in Figure 25 as a result of accounting for the differences in test conditions. The mean square of 17.1 in Figure 25 is less than the value of 20.6 in Figure 23.

The theoretical efficiency values for the actual test conditions are shown for collector No. 2 in Figure 26. Here, as for all the collector No. 2 curves, the combination of test conditions and collector performance characteristics result in a second-order curve with a slightly decreasing slope. The scatter resulting from different test environments is again substantial but does not fully account for the scatter observed in Figure 14. The theoretical efficiency curve at "reference" conditions is shown in Figure 27.

Applying the corrections to the efficiencies shown in Figure 14, the result for collector No. 2 is shown in Figure 28. The mean square is reduced from 17.5 to 13.5 in accounting for different environmental and test conditions. Many of the points that are farther away from the best-fit curve 
still appear in Figure 28. Restricting the results to those tests which reportedly met ASHRAE 93-77 conditions, Figure 29 shows the theoretically justifiable scatter. Figure 30 shows the corresponding corrected results from the seven organizations. Comparing Figure 28 with Figure 30, many, but not all, of the larger differences are eliminated. The mean square is reduced from 8.5 in Figure 16, to 7.2 in Figure 30. A large reduction in scatter is observed in simply going from Figure 14, with a mean square of 17.5, to Figure 16 with 8.5. On the other hand, the mean square for the corrected results in Figure 28 is only about 23 percent lower than for Figure 14.

Figures 16 and 30 show that the efficiencies reported by participant 0 are significantly lower than those reported by the other six participants. Figures 31 through 33 show the consensus of the results from the other six organizations. The mean square is substantially reduced. The correction process reduces the mean square from 4.6 in Figure 31 to 4.0 in Figure 33. The best correlation obtained in the study, without arbitrarily omitting results, is that shown in Figure 33.

Linear best-fit curves, corresponding to the four principal figures, are shown in Figure 34-37. These curves are included so that the intercepts and slopes can be compared with the results obtained from all 21 organizations.

Table 4 is a summary of the pertinent statistical information for linear and second-order least-squares fits to the various sets of efficiencies.

In conducting this analysis, it could not be concluded which of the two limiting cases that were considered best accounts for the effects of scattered radiation. Assuming scattered radiation originates from a direction near the sun, the cover transmittance is the same for beam and scattered radiation. In the other limiting case, scattered radiation was considered completely diffuse and a correction was applied. However, the overall effects on efficiencies resulting from these corrections were considerably less than the experimental uncertainties.

Attempts to investigate the sensitivity of the correlations to sky temperatures were also inconclusive. Sky temperatures of ambient minus $6^{\circ} \mathrm{C}$ for summer and ambient minus $20^{\circ} \mathrm{C}$ for winter [6] were used in place of equation (6) of Appendix C. The mean squares changed by less than 1.0 when compared to the corresponding plots using equation (6). Although sky temperatures may have a significant effect on measured efficiency, the effect could not be identified from the information available.

An attempt was made to test the adequacy of the correlations for wind, percent scattered, etc. It was not possible to evaluate the individual correlations because of the relatively large amount of scatter in the results after all other corrections had been made.

The effect of different techniques for mounting collectors on test frames, which would change the back and side insulation, was considered. Again, no general trends could be associated with the mounting technique. 
In general, the results from the collector No. 1 tests show more scatter than the results from the later collector No. 2 tests. The correction procedure, however, reduced the amount of scatter more for the former test results. Applying corrections progressively, each effect considered either reduced the mean square or resulted in a change too small to determine a definite trend. Individual large deviations from the best-fit curve were reduced significantly. The many points clustered closer, however, were changed with less consistent trends. The combined effect resulted in changes of the mean square which were small. The scatter remaining after the corrections were applied is attributed primarily to experimental and data reduction errors and will be addressed in Section 7. However, the limitation of the correction theory and the possibility of differences between the 12 collectors must be considered. It is also recognized that normal outdoor weather can have an adverse effect on measuring and recording apparatuses.

Figures 38 and 39 show, for the two collectors used in the round robin program, theoretical curves for more favorable and less favorable combinations of environmental conditions. These hypothetical limiting-case combinations are based on conditions and ranges allowed by ASHRAE 93-77. The graphs are included to show the importance of referencing efficiencies to a common environment. The approach developed herein could be extended and verified to apply more generally to flat-plate collectors.

\section{Analysis of The Effect of Random and Systematic Errors}

As shown in Section 6 of this report, there was considerable scatter in the data when considered as a combined group (i.e., Figures 13 and 14); however, the data for an individual facility were generally consistent and showed very little scatter. In addition, correcting the data for the different environmental conditions that existed from facility to facility was shown to reduce the scatter between facilities by less than $30 \%$. Therefore, one concludes that either the requirements of the test are not restrictive enough or that there were systematic errors which were not accounted for by the various participants. In order to examine those possibilities, another round robin participant, B.D. Wood of Arizona State University, conducted the analysis reported in this Section.

The data taken during a given test are recorded and reduced to a plot of thermal efficiency, $n$, versus the parameter $\left(t_{f, i}-t_{a}\right) / I$, here called $x$. Each measurement has associated with it a random or uncertainty error and a systematic error such that (the specific heat is assumed to be known exactly):

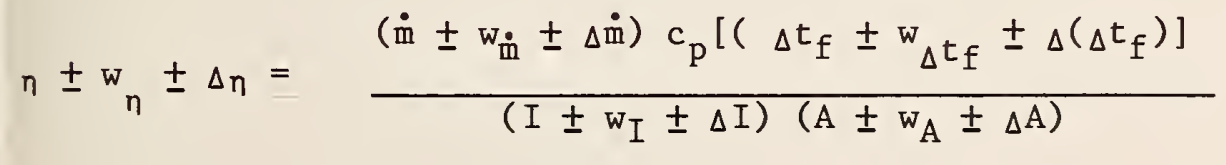




$$
X \pm w_{X} \pm \Delta X=\frac{\left[\left(t_{f, i} \pm w_{t_{f, i}} \pm \Delta t_{f, i}\right)-\left(t_{a} \pm w_{t_{a}} \pm \Delta t_{a}\right)\right]}{\left(I \pm w_{I} \pm \Delta I\right)}
$$

where $\Delta t_{f}=t_{f, e}-t_{f, i}$

$\mathrm{w}=$ random or uncertainty error which could be either positive or negative with the same probability. The sign of $w$ is indeterminant.

$\Delta=$ systematic error. The sign could be either positive or negative and will remain fixed for a given test apparatus (e.g., conduction errors for thermocouple measurement, calibration error for pyranometer, or radiation error for ambient air temperature measurement).

Random errors cause data scatter for a particular test facility. Systematic errors cause a disagreement between test facilities. Since there is relatively small scatter (less than $4 \%$ ) for a given test facility, it is reasonable to assume that the discrepancies among the combined data are due primarily to systematic errors at the facilities. However, if the test specifications were not tight enough, similar discrepancies could occur.

First consider the scatter that could be attributed to the experimental uncertainty in the specifications of the test in [1,2] and [4]. The maximum uncertainty or error allowable is:

$$
w_{\eta}= \pm \frac{\partial \eta}{\partial \dot{m}} w_{\dot{m}}+\frac{\partial \eta}{\partial \Delta t_{f}} w_{\Delta t_{f}}+\frac{\partial \eta}{\partial I} w_{\dot{I}}+\frac{\partial \eta}{\partial A} w_{A}
$$

or,

$$
\frac{w_{n}}{\eta}= \pm \frac{w_{m}^{0}}{\underline{m}}+\frac{{ }^{w}}{\Delta t_{f}} f+\frac{{ }^{w_{I}}}{I}+\frac{{ }^{w_{A}}}{A}
$$

Similarly,

$$
\frac{w_{X}}{X}= \pm \frac{{ }_{t_{f, i}}+{ }_{t}{ }_{t}}{\left(t_{f, i}-t_{a}\right)}+\frac{W_{I}}{I}
$$

The allowable uncertainty or inaccuracy in the measurements as specified in or estimated from [1,2] and [4] are such that: 


$$
\begin{aligned}
& \frac{w_{\dot{m}}}{m}= \pm 0.01 \\
& { }^{\mathrm{w}} \Delta \mathrm{t}_{\mathrm{f}}= \pm 0.1^{\circ} \mathrm{C} \\
& \frac{{ }^{\mathrm{W}} I}{\mathrm{I}}= \pm\left(0.03+\frac{0.1}{\mathrm{I}}\right) \\
& \frac{{ }^{\mathrm{W}} \mathrm{A}}{\mathrm{A}}= \pm 0.01 \\
& \mathrm{w}_{\mathrm{t}_{\mathrm{f}, \mathrm{i}}}= \pm 0.5^{\circ} \mathrm{C} \\
& \mathrm{w}_{\mathrm{t}_{\mathrm{a}}}= \pm 0.5^{\circ} \mathrm{C}
\end{aligned}
$$

Therefore

$$
\begin{aligned}
& \frac{w_{n}}{n}= \pm\left(0.05+\frac{0.1}{\Delta t_{f}}+\frac{0.1}{I}\right) \\
& \frac{w_{X}}{x}= \pm\left(0.03+\frac{1.0}{t_{f, i}-t_{a}}+\frac{0.1}{I}\right)
\end{aligned}
$$

From $[1,2]$ and [4], I $\geq 630 \mathrm{~W} / \mathrm{m}^{2}$, therefore equations (18) and (19) become:

$$
\begin{aligned}
& \frac{w_{n}}{n} \leq \pm\left(0.0502+\frac{0.1}{\Delta t_{f}}\right) \\
& \frac{{ }^{w} X}{X} \leq \pm\left(0.0302+\frac{1.0}{t_{f, i}-t_{a}}\right)
\end{aligned}
$$

Considering collector No. 2, the following typical test values are taken from Tables 1 and $\mathrm{C} 1$ :

$$
\begin{aligned}
& \mathrm{A}=1.79 \mathrm{~m}^{2} \\
& \dot{\mathrm{m}}=0.0358 \mathrm{~kg} / \mathrm{s} \\
& \mathrm{c}_{\mathrm{p}}=4187 \mathrm{~J} /\left(\mathrm{kg} \cdot{ }^{\circ} \mathrm{C}\right) \\
& \mathrm{I} \geq 630 \mathrm{~W} / \mathrm{m}^{2} \\
& \mathrm{t}_{\mathrm{a}}=20^{\circ} \mathrm{C}
\end{aligned}
$$

Then, and noting that $\Delta t_{f}$ can be calculated by:

$$
\Delta t_{f}=\frac{\eta I A}{\dot{m} c_{p}}
$$




$$
\begin{aligned}
& \Delta t_{f} \geq 7.523 n \\
& t_{f, i} \geq 20+630 x
\end{aligned}
$$

and

$$
\begin{aligned}
& \frac{w_{n}}{\eta} \leq \pm\left(0.0502+\frac{0.10}{7.523 \eta}\right) \\
& \frac{w_{x}}{x} \leq \pm\left(0.0302+\frac{1.0}{630 x}\right)
\end{aligned}
$$

Equations (25) and (26) can be used to define the "maximum error" band for the test data for collector No. 2. For example, the data in Figure 37 can be correlated by:

$$
\eta=0.814-4.98 x
$$

Then for each value of $X$ and corresponding $n$, the maximum error band allowable from references [1, 2] and [4] can be calculated as follows:

\begin{tabular}{lccc}
$x$ & 0.01 & 0.05 & 0.10 \\
\hline$n$ & 0.764 & 0.565 & 0.316 \\
$\frac{w_{n}}{n}$ & $\pm 0.068 \pm 0.074$ & \pm 0.092 \\
${ }^{w_{n}}$ & $\pm 0.052 \pm 0.042$ & \pm 0.029 \\
$\frac{{ }^{W}}{X}$ & \pm 0.189 & 0.062 & 0.046 \\
${ }^{w_{X}}$ & $\pm 0.0019 \pm 0.0031$ & \pm 0.0046
\end{tabular}

Figure 40 is a replot of Figure 36 where all the uncorrected data for, collector No. 2 for the 10 selected participants is shown along with the maximum error band defined above. Figure 41 is a similar plot but for only those participants which reported adhering strictly to the requirements of ASHRAE Standard 93-77. Figures 42 and 43 are corresponding plots after the data have been corrected to "reference" conditions.

Since the data of Figures 41 and 43 all lie within the this error band, one might conclude that the scatter was due entirely to allowable uncertainty as specified in references [1, 2] and [4] and no systematic error existed at the facilities shown in Figures 41 and 43 . It can more logically be assumed that the data scatter among the various test facilities taken as a group would most probably follow some sort of randomness and be 
considerably less than the maximum allowable error band. Therefore, a second analysis was done.

A numerical experiment was conducted to determine the "anticipated" data scatter from 10 hypothetical test facilities, each claiming compliance with ASHRAE 93-77. Table 5 is a matrix of possible errors associated with each measurand. The probability of each error is the same (this is conservative compared to a normal probability distribution about the mean). A UNIVAC library program was used to generate a random number between 0 and 1.0. This number with a corresponding error selected from Table 5 for each measurand at the ten hypothetical facilities was then used to select the appropriate error. Hence, 60 random numbers between 0 and 1.0 were required to generate Table 6 , which is a tabulation of errors assigned with each measurement at each

of the 10 hypothetical test facilities. The collector computer model described in Appendix $C$ was used to obtain an efficiency curve for collector No. 2. This curve was then used to calculate the temperature rise across the collector for a given collector inlet temperature at the following conditions:

$$
\begin{aligned}
\mathrm{I} & =1000 \mathrm{~W} / \mathrm{m}^{2} \text { or } 630 \mathrm{~W} / \mathrm{m}^{2} \\
\mathrm{~A} & =1.79 \mathrm{~m}^{2} \\
\dot{\mathrm{m}} & =0.0358 \mathrm{~kg} / \mathrm{s} \\
\mathrm{t}_{\mathrm{a}} & =20^{\circ} \mathrm{C} \\
\mathrm{t}_{\mathrm{f}, \mathrm{i}} & =20,30,40,50, \ldots{ }^{\circ} \mathrm{C}
\end{aligned}
$$

Then in order to calculate the supposedly measured efficiencies for each collector at the 10 test facilities, the following relationships were used:

$$
\begin{aligned}
\mathrm{I}_{j} & =1000+\Delta \mathrm{I}_{j} \text { or } 630+\Delta \mathrm{I}_{j} \\
\mathrm{~A}_{j} & =1.79+\Delta \mathrm{A}_{j} \\
\dot{\mathrm{m}}_{j} & =0.0358+\Delta \dot{\mathrm{m}}_{j} \\
\mathrm{t}_{\mathrm{a}_{j}} & =20+\Delta \mathrm{t}_{\mathrm{a}_{j}} \\
\mathrm{t}_{\mathrm{f}, \mathrm{i}_{j, k}} & =\mathrm{t}_{f, i_{k}}+\Delta \mathrm{t}_{f, i_{j}} \\
\Delta \mathrm{t}_{j, k} & =\Delta \mathrm{t}_{\mathrm{k}}+\Delta \Delta \mathrm{t}_{j}
\end{aligned}
$$

These supposedly measured efficiencies are shown in Figures 44 and 45 for an insolation of $630 \mathrm{~W} / \mathrm{m}^{2}$ and $1000 \mathrm{~W} / \mathrm{m}^{2}$, respectively. As expected, the scatter is considerably less than the "maximum" error band also shown in the Figures. By comparing Figures 43 and 45 , one concludes that the data scatter that 
actually occurred (after the data were corrected for differences in operating and environmental conditions) among those facilities that reportedly adhered to ASHRAE Standard 93-77 was much larger than should be expected. Also notice that the combined errors in the numerical experiment only shifted the curve up or down without changing the slope. Therefore, systematic facility errors undoubtedly existed. Unfortunately, an evaluation of the experimental apparatuses and various experimental techniques of the various participants was not performed.

Each participant was provided with a copy of the test procedure which describes the accuracy, precision and calibration requirements for each measurement. However, meeting these requirements does not preclude the possibility of uncertainties arising from sensor installation, data acquisition, or data reduction.

Based on the data available, it is only possible to hypothesize that some combination of the following systematic errors caused a portion of the data scatter:

\section{Systematic Facility Errors:}

- heat transfer between the test apparatus and the collector (proximity of heat sources to the collector)

- reduced heat losses to ambient due to shielding of collector support stand

- test apparatus not at steady state or "quasi-steady-state" conditions during tests

- change in specific heat of the transfer fluid

- inexperienced technicians conducting the tests.

Systematic Instrumentation Errors:

- conduction errors in thermocouple installations

- pyranometer calibration error

- flow meter calibration error

8. Implication of Collector Performance Uncertainty on System Performance

The significance of the variation in the reported performance for a particular collector can be illustrated by calculating the expected system performanct for two sample solar systems. 
For the first example, the performance was calculated for a typical combined solar domestic hot water and space heating system. A residence located in Madison, Wisconsin with a design heat loss of $21 \mathrm{~kW}$ was selected. A hot water demand of $0.32 \mathrm{~m}^{3}$ per day combined with the space heating load resulted in an average monthly energy requirement for the year of about $1.7 \times 10^{9} \mathrm{~J}$. The solar energy system assumed consisted of $80 \mathrm{~m}^{2}$ of collector No. 1. Other system features included a heat exchanger between the collector and storage, an insulated $3.8 \mathrm{~m}^{3}$ liquid storage tank, a domestic hot water heat exchanger and preheat storage tank with suitable pumps and controls. Auxiliary energy was supplied when sufficient energy was not available from thermal storage.

The calculation of the fraction of the load supplied by solar energy was performed using the f-chart method [9]. The collector thermal performance factors used for $F^{\prime}(\tau \alpha)_{e}$ and $F^{\prime} U_{L}$ are listed in Table 4 for the mean, "best" and "worst" values as reported in section 5 of this report. The results show a deviation of +10.3 and -11.8 percentage points in yearly solar fraction from the mean value or a total spread of 22.1 percentage points.

A second sample problem was completed again using the f-chart technique. A solar domestic hot water system was simulated in five different cities across the country. For this calculation, it was assumed that $5.37 \mathrm{~m}^{2}$ of collector No. 2 without a heat exchanger were used. The relationship between $F^{\prime}$ and $F_{R}$ was taken from reference [6]. The collector was assumed tilted at an angle equal to the latitude and the system used a $0.45 \mathrm{~m}^{3}$ storage tank. The system supplied hot water at a constant temperature of $60^{\circ} \mathrm{C}$ with a constant inlet temperature which varied from city to city as shown in Table 8 and 9. The system was simulated using the collector performance factors from all 10 test facilities identified in Section 6 of this report. Table 8 shows the calculated yearly fraction of the hot water load supplied by solar energy using data from the six facilities adhering to the requirements of ASHRAE Standard 93-77. The maximum spread is 6.5 percentage points for Washington, D.C. Table 9 shows the results using data from test facilities reporting the five highest and the five lowest performance. Again the maximum spread occurs in Washington, D.C., 17.8 percentage points.

\section{Conclusions}

The results of the analysis indicate that the majority of the reported differences in measured collector efficiency in the round robin testing program resulted from experimental error or systematic differences from facility to facility rather than from differences in the outdoor test environments.

The preparation and mounting of the collector for test appeared to cause a large uncertainty in test results for collector No. 1.

The use of a pyranometer meeting the World Meteorological Organization Class I requirements and a pyranometer calibrated for use at test tilt angles was found to be of major importance. 
The environment can have a significant effect on the measured collector efficiency even when conditions prescribed by current standards are met. Within the limitation of the accuracy of flat-plate collector performance theory, it was found that test results could be referenced to a common environment using a straightforward analytical procedure. The procedure significantly reduced the differences in measured efficiencies reported by the various participants.

Efficiency correlations from individual participants generally showed small scatter. Larger differences were observed, however, between efficiencies measured by various participants at approximately the same conditions. The second series of tests (on collector No. 2) gave more consistent results than the first.

Tests that reportedly met the more restrictive ASHRAE 93-77 requirements showed much more consistency among participants. It was not apparent, however, whether the improvement resulted from the additional restrictions or better experimental procedures by those participants. By restricting efficiency measurements for collector No. 2 to 6 of the 7 participants reportedly meeting ASHRAE 93-77 requirements, highly consistent results were observed.

It is felt that outdoor solar collector testing will continue to be performed at an ever increasing rate. The technique of correcting test results to "reference" conditions is a potentially useful aid for the solar collector industry. The technique should be validated and then packaged into a useful tool that could easily be utilized within the industry. In addition, procedures need to be adopted to ensure facility-to-facility agreement. Continuing experiments would be desirable where two or three "independent" facilities would conduct a series of tests and insure intercomparability. The facilities would be picked so that extremes in environmental conditions could be studied. The tests might include:

- interchanging collectors

- determining the effect of environmental conditions on data scatter and reproducibility (wind, sky temperatures, insolation level, percent diffuse, ambient temperature)

- intercomparison of pyranometers

- determining the spectral distribution of the radiation for the three locations

- determining when steady conditions occur for thermal testing. 
1. Hill, J.E., and T. Kusuda, "Methods of Testing for Rating Solar Collectors Based on Thermal Performance", NBSIR 74-635, December, 1974.

2. Hi11, J.E., Streed, E.R., Kelly, G.E., Geist, J.C., and T. Kusuda, "Development of Proposed Standards for Testing Solar Collectors and Thermal Storage Devices", NBS Technical Note 899, February, 1976.

3. Hill, J.E., and E.R. Streed, "A Method of Testing for Rating Solar Collectors Based on Thermal Performance", Solar Energy, Vol. 18, No. 5, 1976.

4. "Methods of Testing to Determine the Thermal Performance of Solar Collectors", ASHRAE Standard 93-77, ASHRAE, 345 East 47th Street, New York, New York 10017, 1977.

5. Hottel, H.C., and B.B. Woertz, "The Performance of Flat-Plate Solar Heat Collectors", ASME Transactions, Vol. 64, p. 91, 1942.

6. Duffie, J.A., and W.A. Beckman, Solar Energy Thermal Process, John Wiley and Sons, New York, 1974.

7. Latimer, J.R., "Radiation Measurement", Technical Manual Series No. 2, Canadian National Committee for the International Hydrological Decade, Building No. 8, Carling Avenue, Ottawa, Canada, 1971.

8. Guide to Meteorological Instrumentation and Observing Practices, Secretariat of the World Meteorological Organization, Geneva, Switzerland, 4th Edition, 1971.

9. Klein, S.A., Beckman, W.A., and J.A. Duffie, "A Design Procedure for Solar Heating Systems", Solar Energy, Vol. 18, No. 2., 1976.

10. Klein, S.A., Beckman, W.A., and J.A. Duffie, "A Design Procedure for Solar Air Heating Systems", Solar Energy, Vol. 19, No. 5, 1977.

11. Yass, K., and H.B. Curtis, "Low-Cost Air Mass 2 Solar Simulator", NASA TM X-3509, presented at the United States Section Meeting of the International Solar Energy Society, held in Cleveland, Ohio, October 3-4, 1973.

12. Simon, F.F., and P. Harlamert, "Flat-Plate Collector Performance Evaluation, the Case for a Solar Simulator Approach", NASA TM X-71427, presented at the United States Section Meeting of the International Solar Energy Society, held in Cleveland, Ohio, October 3-4, 1973.

13. Ramsey,-J.W., Borzoni, J.T., and T.H. Holland, "Development of FlatPlate Collectors for Heating and Cooling of Buildings", NASA CR-134804, June, 1975. 
14. Norris, D.J., "Calibration of Pyranometers in Inclined and Inverted Positions", Solar Energy, Vo1. 16, No. 1, 1974.

15. ASTM E178-75, "Standard Recommended Practice for Dealing with Outlying Observations", Annual Book of ASTM Standards, Part 41, ASTM, Philadelphia, Pa., 19103.

16. Thomas, W.C., and A.G. Dawson, III, "Analysis of Data and Results for the Round Robin Flat-Plate Collector Test Program", VPI \& SU Report Eng. 77-23, Virginia Polytechnic Institute and State University, Blacksburg, Virginia, 1977. (NTIS PB-275-576, p. 86).

17. Ostle, B., Statistics in Research, Chapter 6, Iowa State College Press, Ames, Iowa, 1954.

18. Glycols, Union Carbide Corporation, 270 Park Avenue, N.Y. 10017, 1971 .

19. Kays, W.M., "Numerical Solutions for Laminar Flow in Circular Tubes", ASME Transactions, Vo1. 77, p. 1265, 1955.

20. Buchberg, H., Catton, I., and D.K. Edwards, "Natural Convection in Enclosed Spaces - A Review of Application to Solar Energy Collection, ASME Transactions, Vo1. 98C, pp. 184-188, May, 1976.

21. Eckert, E.R.G., and R.M. Drake, Jr., Analysis of Heat and Mass Transfer, p. 65, McGraw-Hil1, N.Y., 1972.

22. Woodman, T.P., "The Effect of Design and Operating Parameters on the Performance of Flat-Plate Solar Collectors-Calculation Method and Detailed Appraisal," Solar Energy, Vol. 19, No. 3, pp. 263-270, 1977.

23. "Baseline Solar Collector," Glass Division, PPG Industries, Inc., One Gateway Center, Pittsburgh, Pa. 15222, 1974.

24. "Technical Data on the Chamberlain Manufacturing Corporation FlatPlate Solar Collector", Chamberlain Manufacturing Corporation, 845 Larch Ave., Elmhurst, Illinois 60126, February, 1976.

25. Bolz, R.E., and G.L. Tuve, Editors, Handbook of Tables for Applied Engineering Science, pp. 441-457, The Chemical Rubber Company, Cleveland, Ohio, 1970. 
Table 1. Pertinent Collector Characteristics

(from the manufacturer's literature)

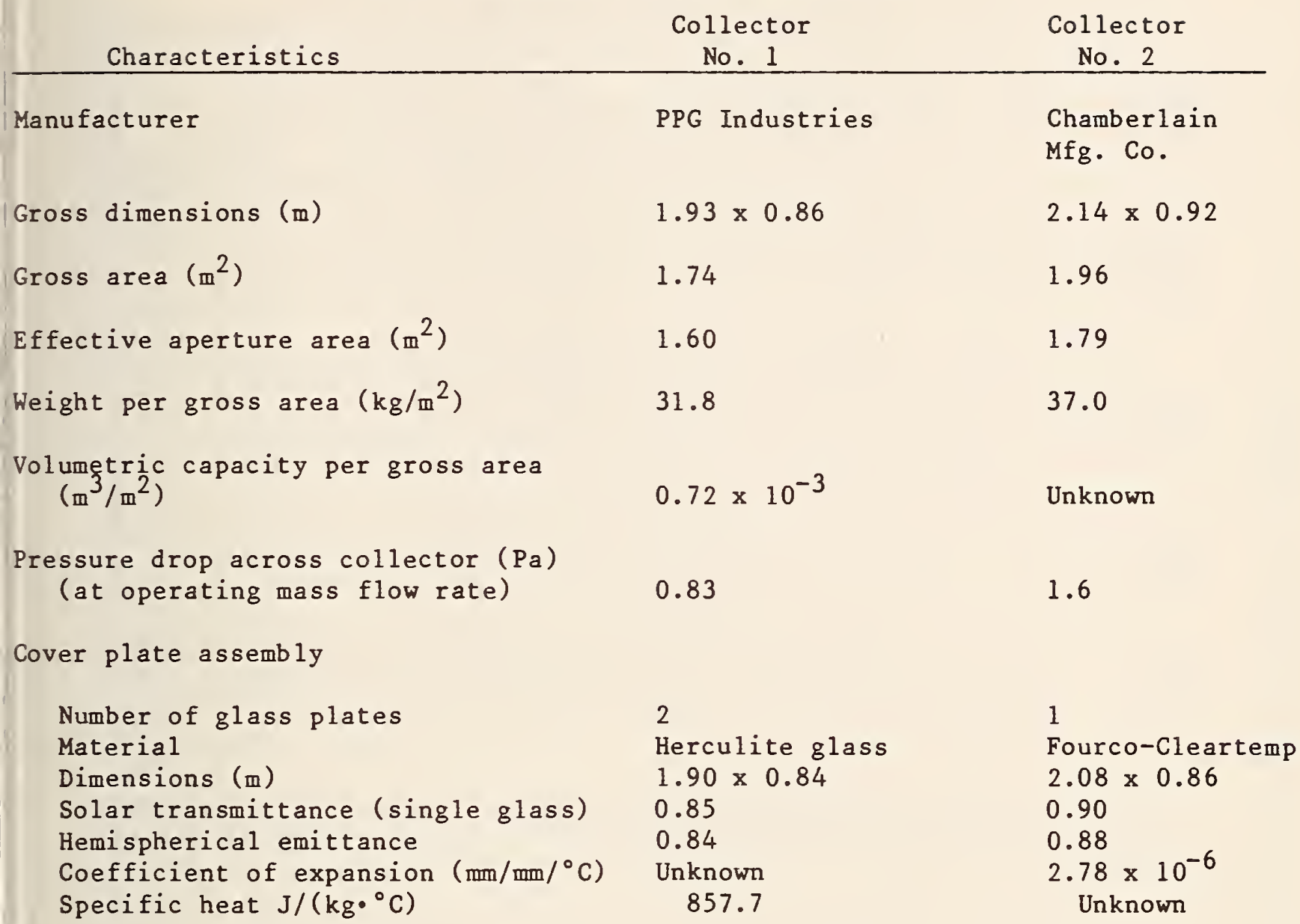

Absorber Plate

Material

Dimensions

Flow configuration

Coating

Solar absorptance

Hemi spherical emittance

Air space(s)

Between covers (mm)

Between cover and absorber (mm)
Alumi num

$1.90 \times 0.84$

13 parallel pass

Durachron 600

L/G flat black

0.94

0.92
Mild stee 1

$2.09 \times 0.86$

19 parallel pass

Black chrome

0.94

0.12

Insulation

Material

Density $\left(\mathrm{kg} / \mathrm{m}^{3}\right)$

Thickness (mm)

Thermal conductivity $\left(\mathrm{W} / \mathrm{m} \cdot{ }^{\circ} \mathrm{K}\right)$
9.53

12.7
19.0
Glass-fiber

48.1

76.2

0.035
Glass-fiber

$\backsim 80$

76.2

Unknown 


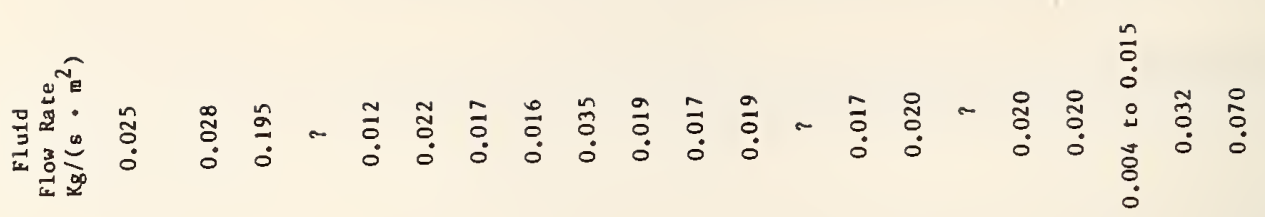

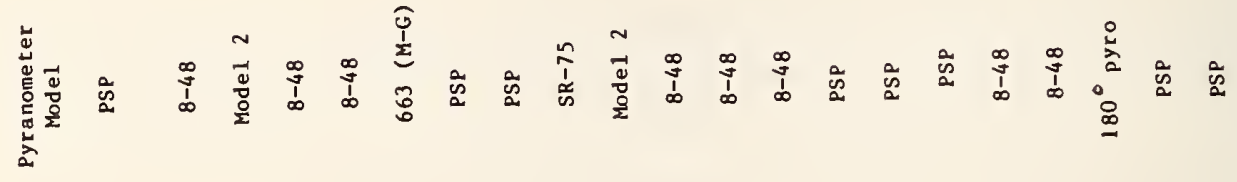

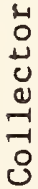

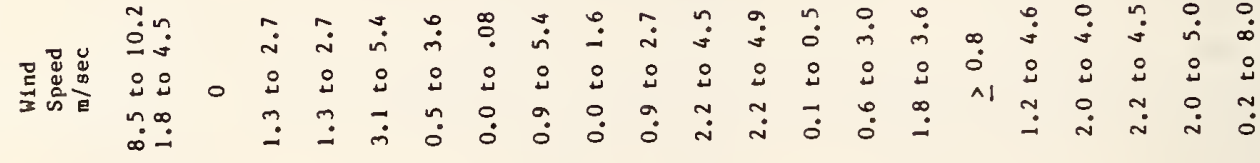

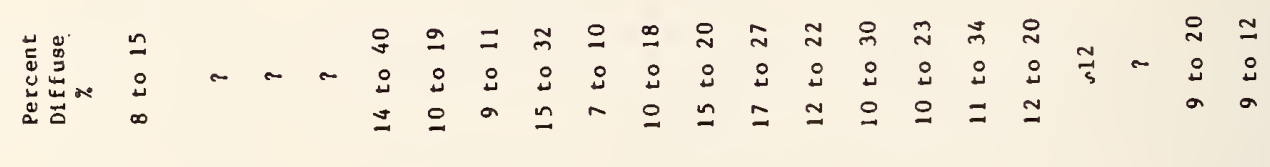

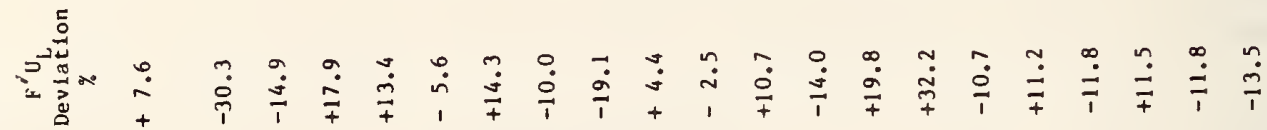

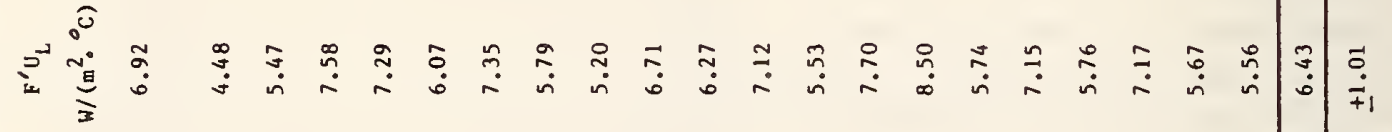
至 4

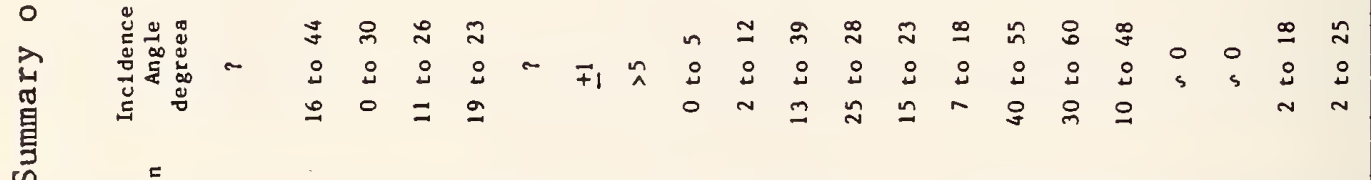

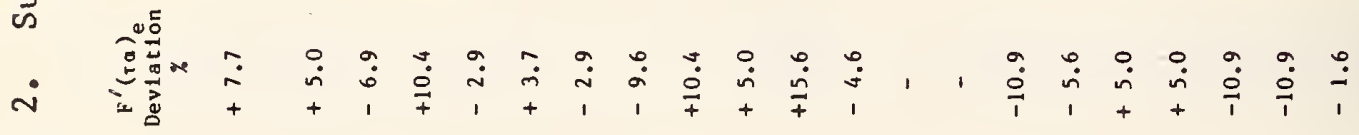

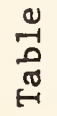

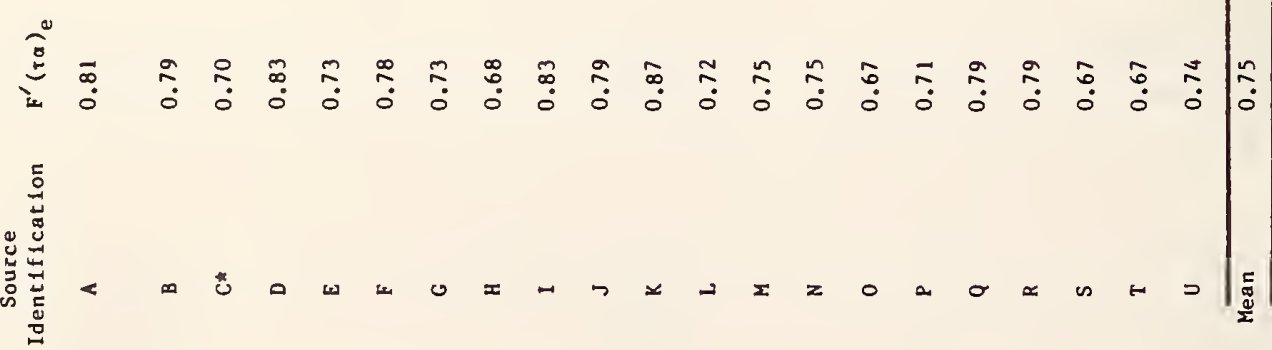

$\square$
0
$\dot{1}$
0
0

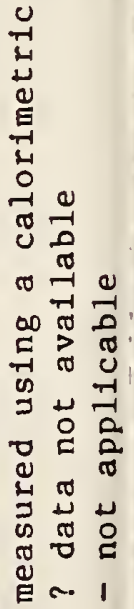




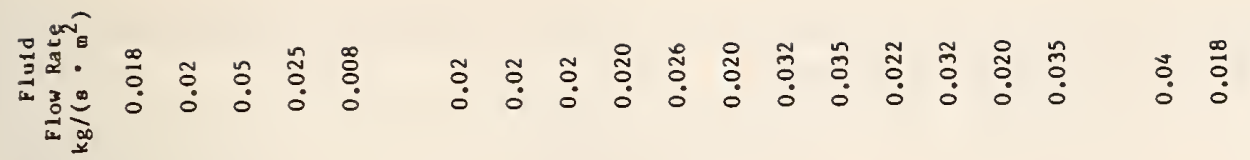

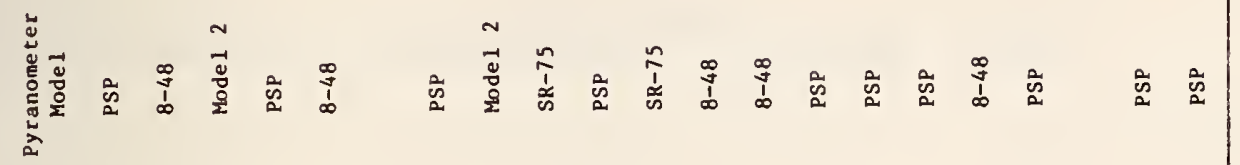
.

sing

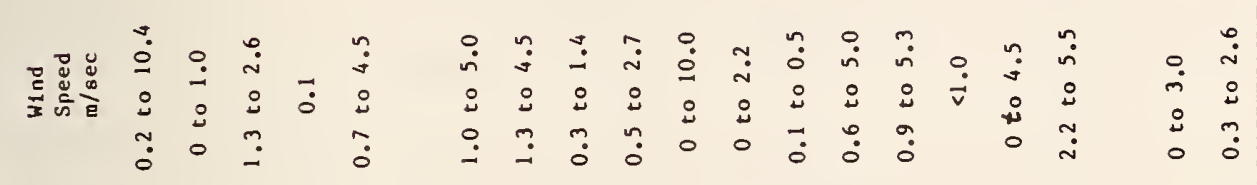

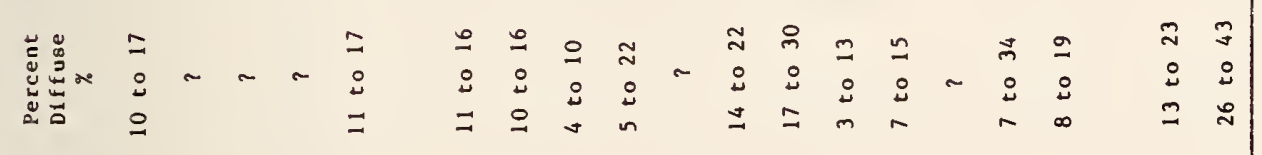

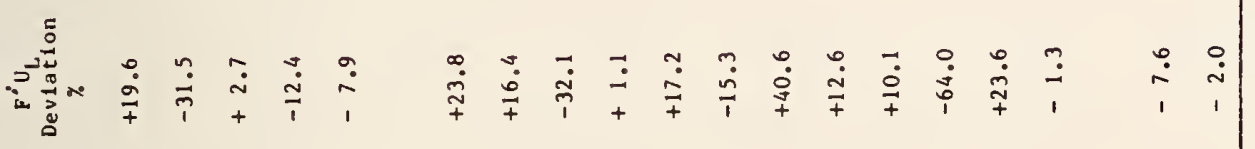

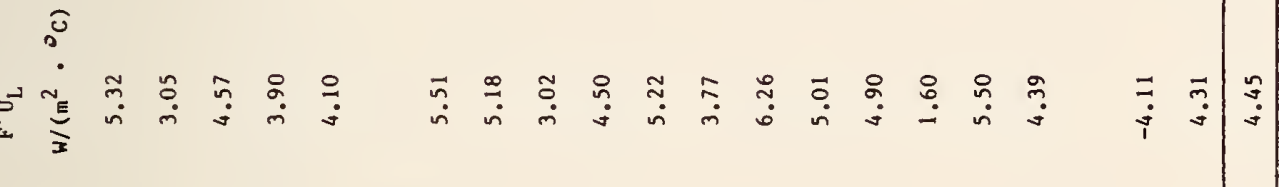

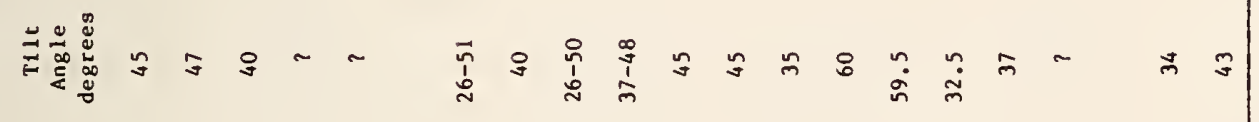

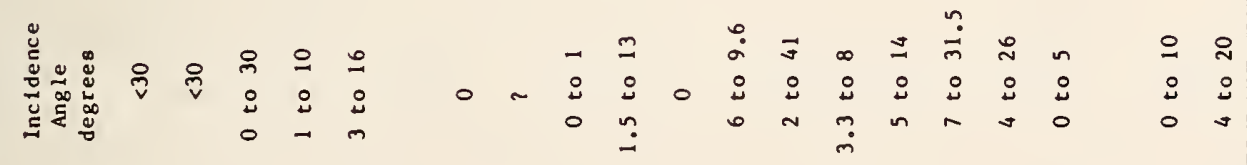

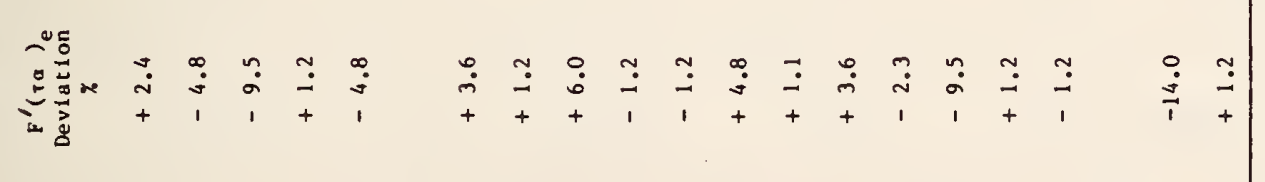

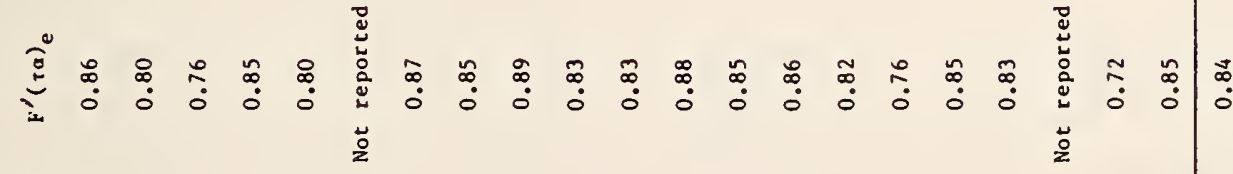

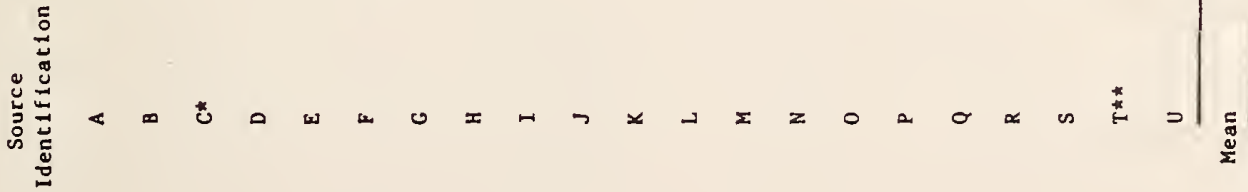

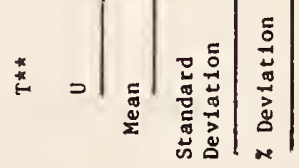


Table 4. Summary of Ordinate-Intercepts, Slopes, and Mean Squares Values for Efficiency Correlations

Efficiency

Set

No. 1 Uncorrected

Corrected

Theoretical

Uncorrected ASHRAE

Corrected ASHRAE

80

80

Theoretical ASHRAE

No. 2 Uncorrected

\begin{abstract}
Corrected
\end{abstract}
Theoretical

Uncorrected ASHRAE

112

112

Corrected ASHRAE

Theoretical ASHRAE 112

Uncorrected ASHRAE

96

96

0.829

96

Theoretical ASHRAl

$w /\left(m^{2} \cdot{ }^{\circ} \mathrm{C}\right)$
Linear Curve

Second-Order

No. of

192

0.705

5.61

30.5

0.731

27.9

92

0.728

5.98

20.6

0.733

20.6

80

0.707

6.30

23.2

0.722

23.2

2.46

0.696

2.4 


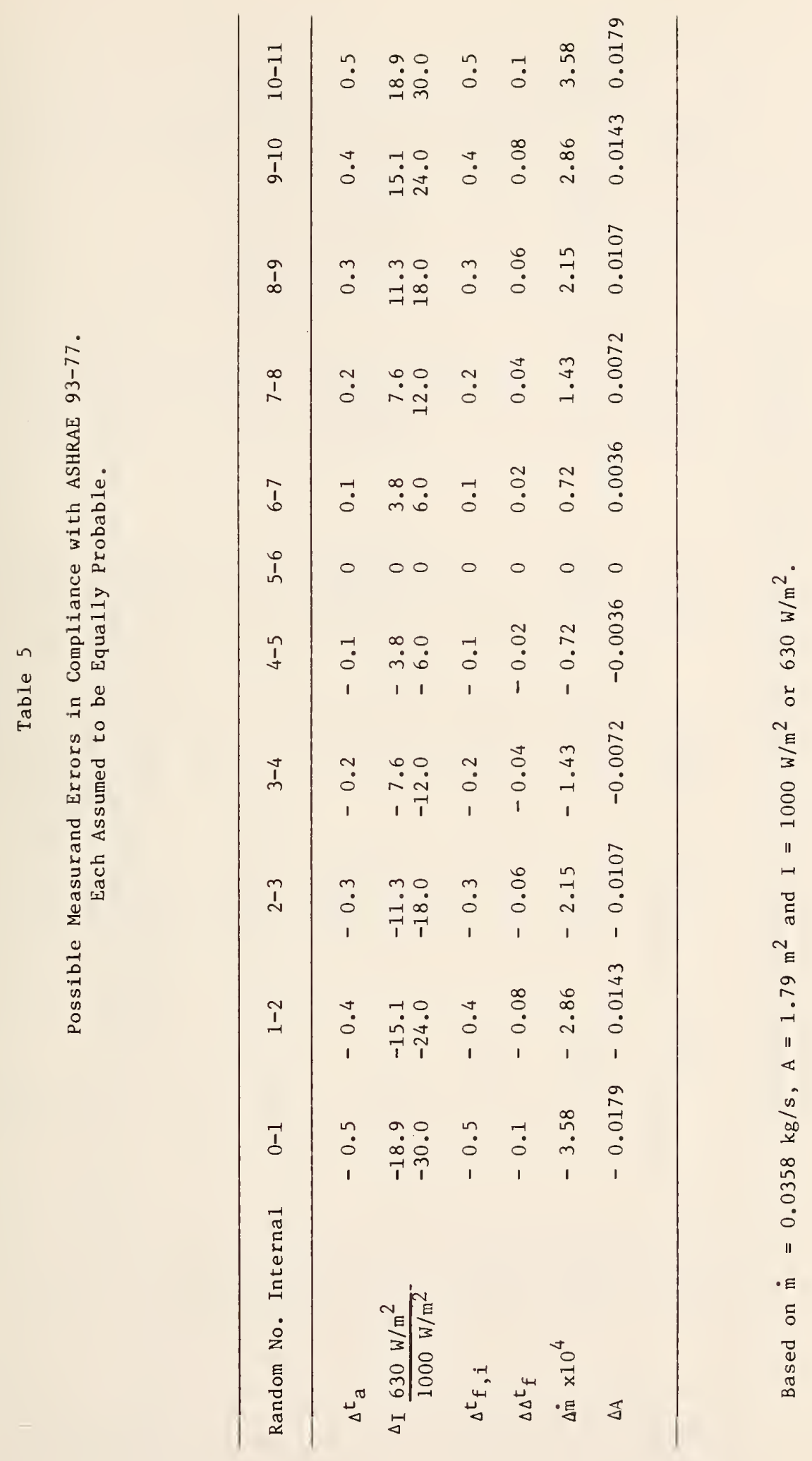




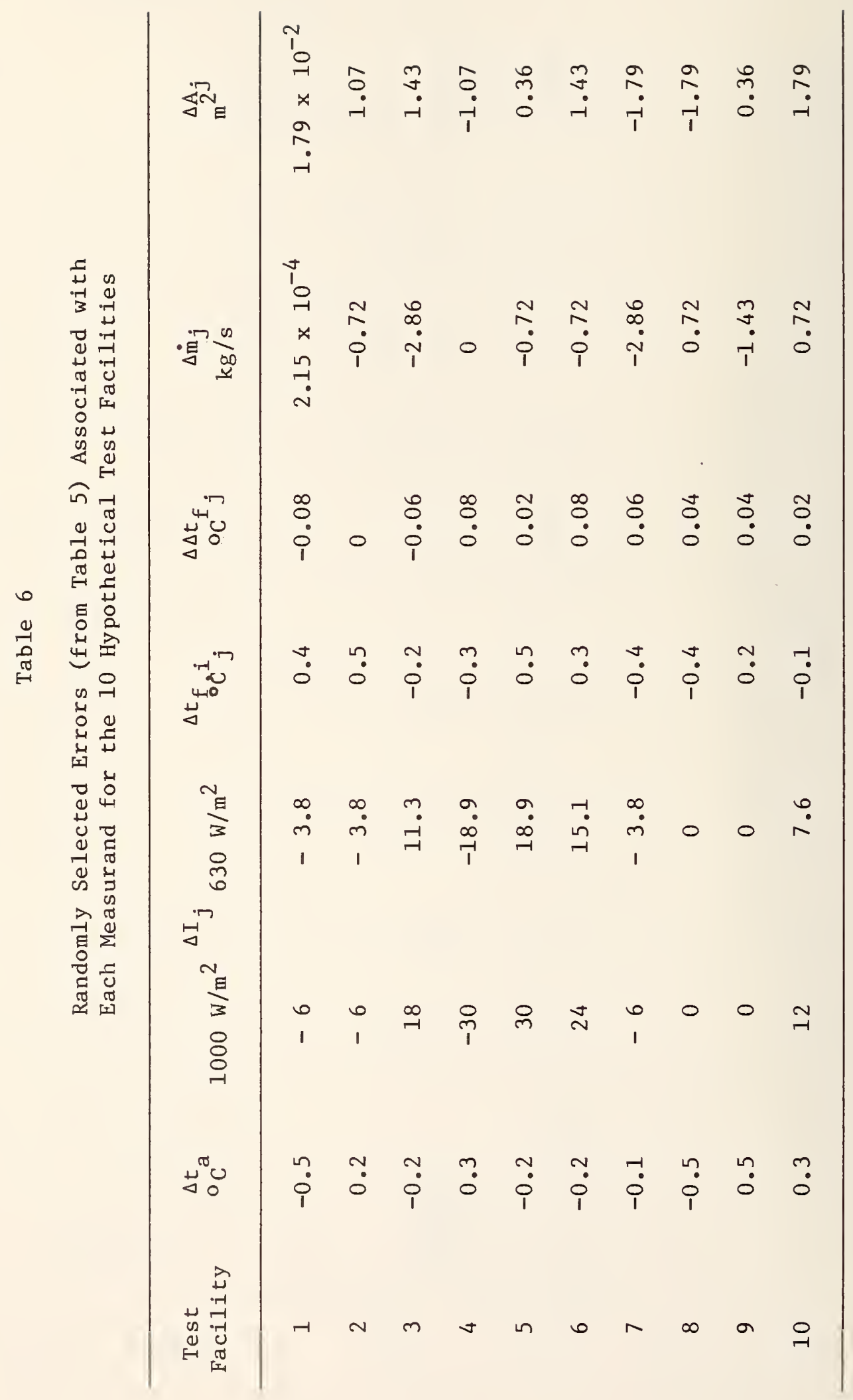




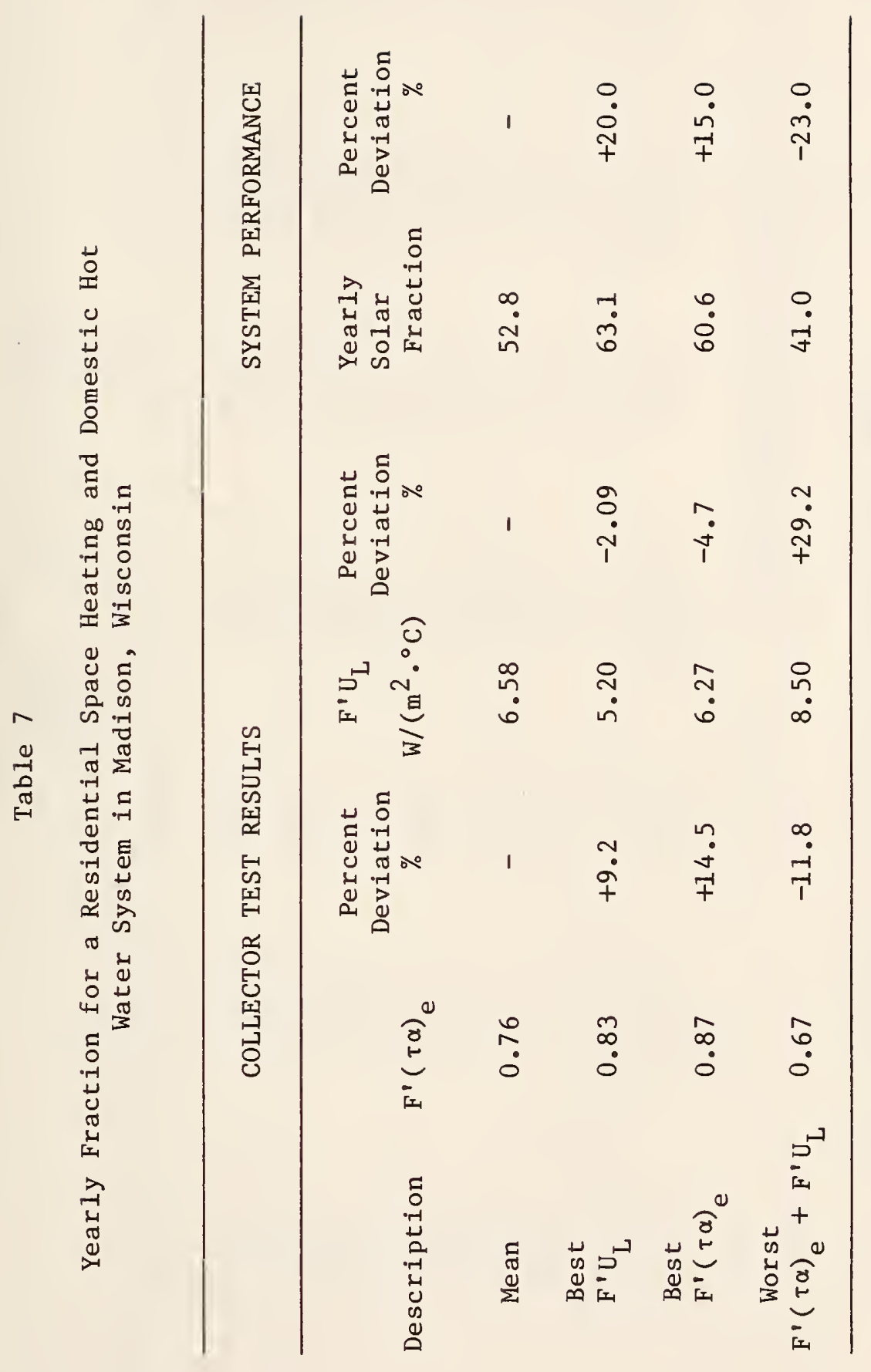




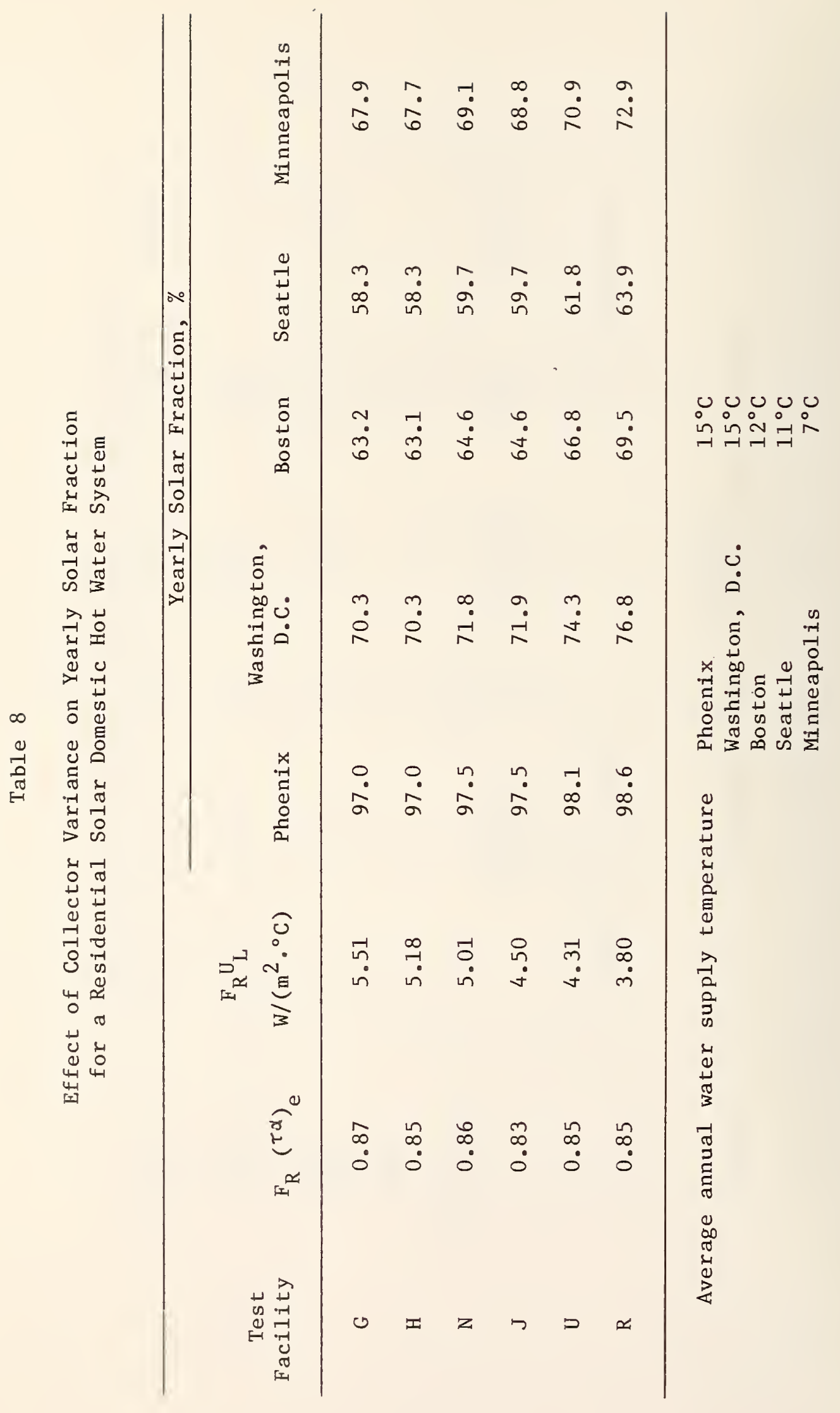




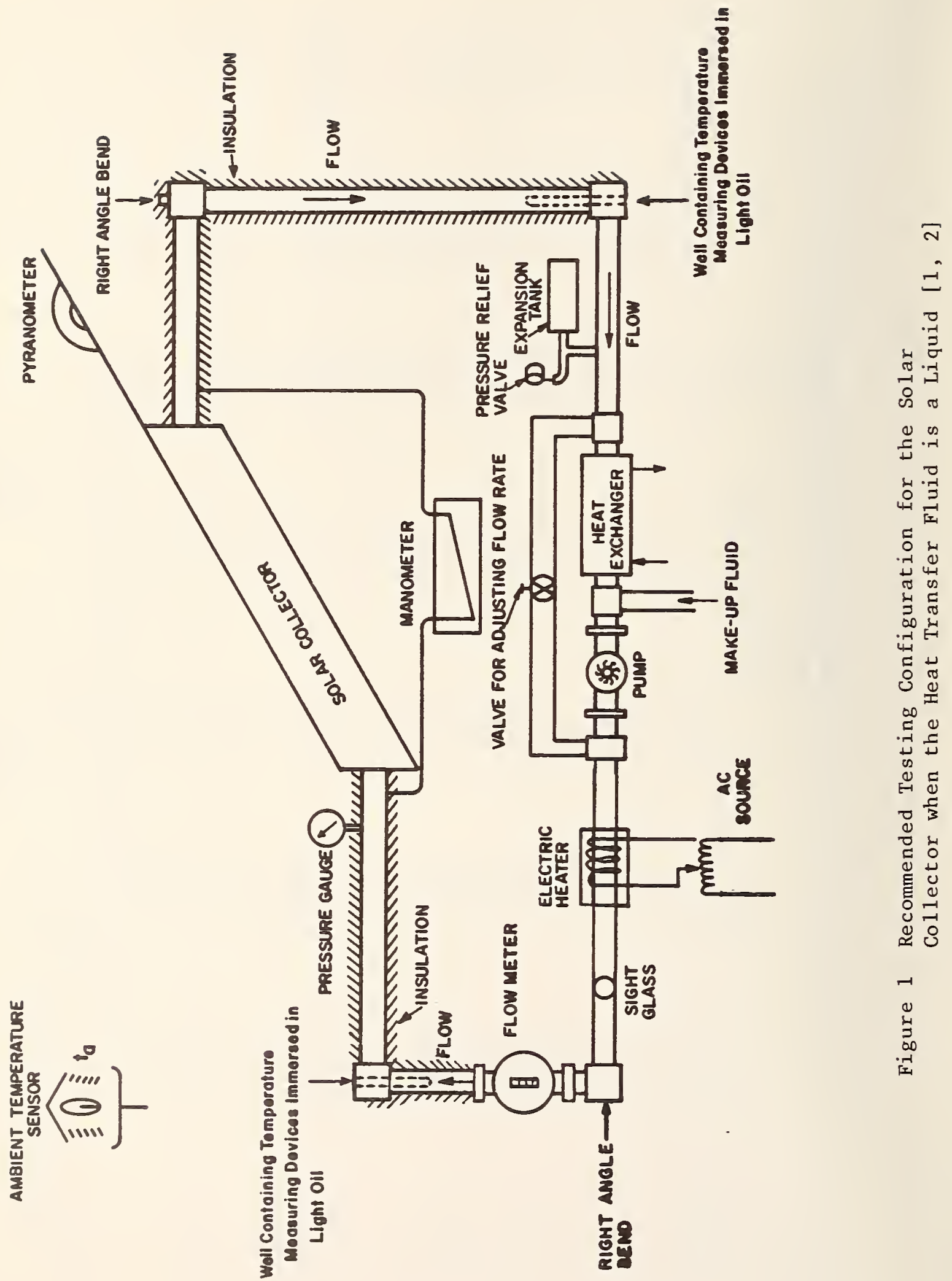




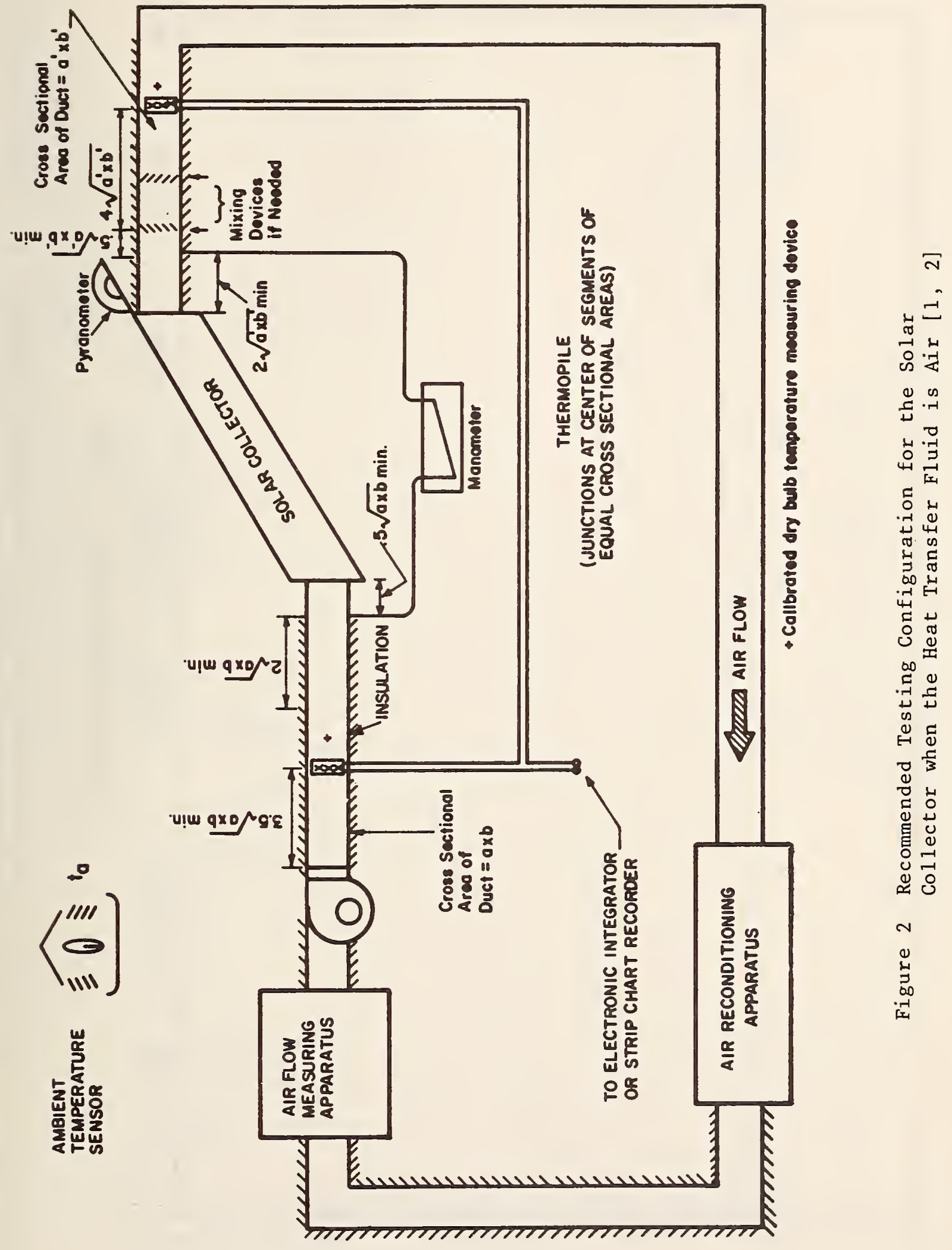




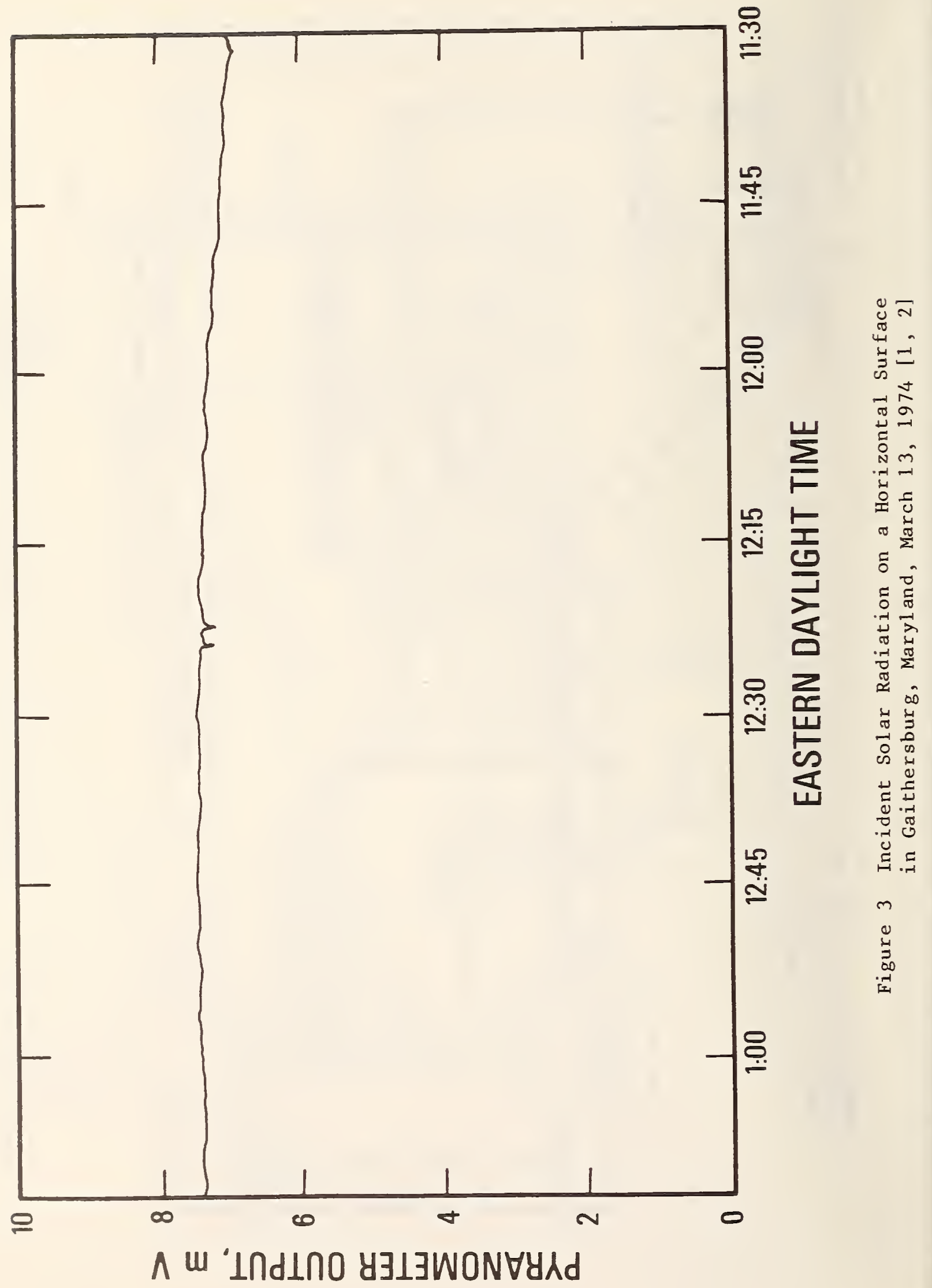




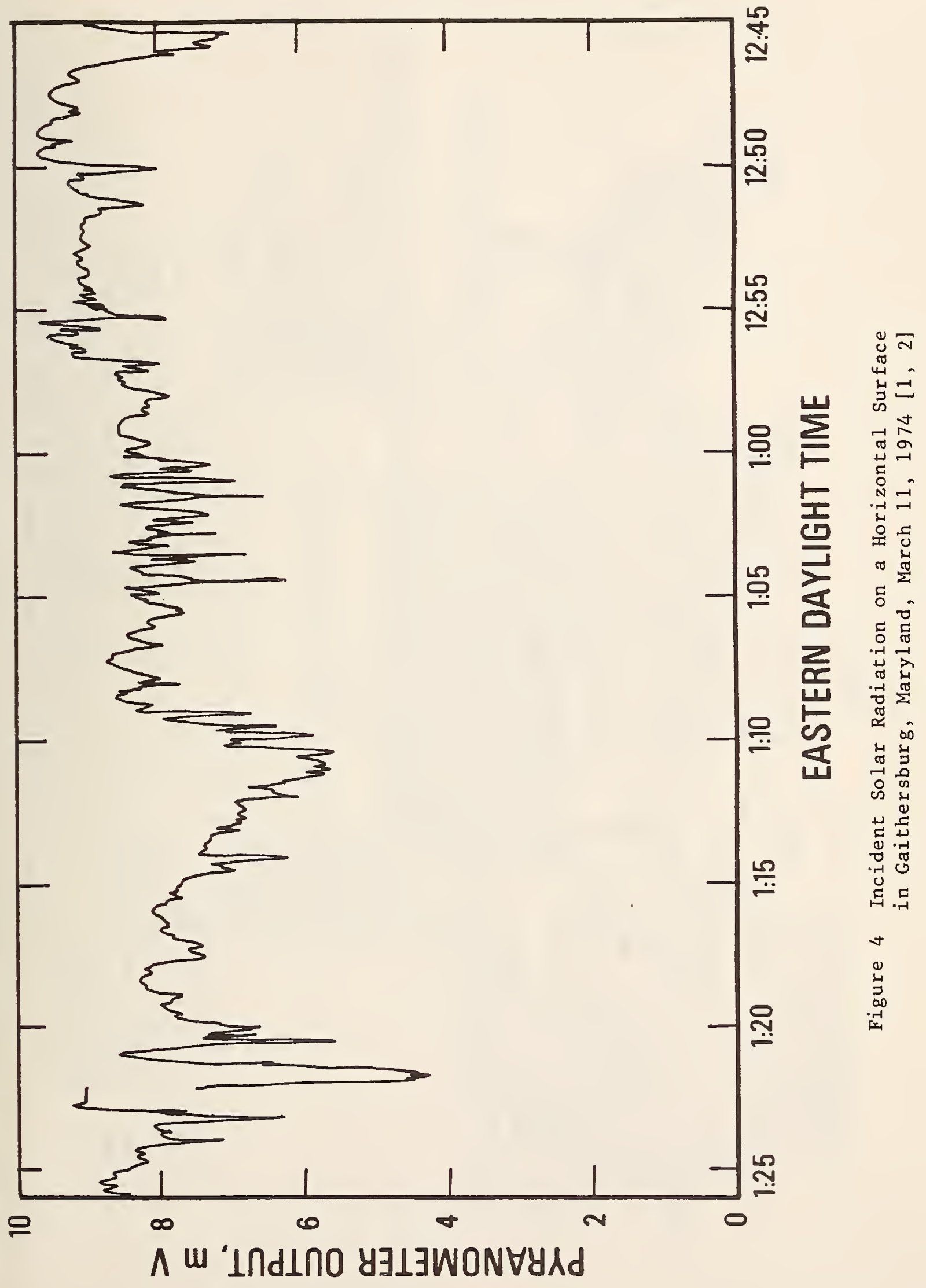




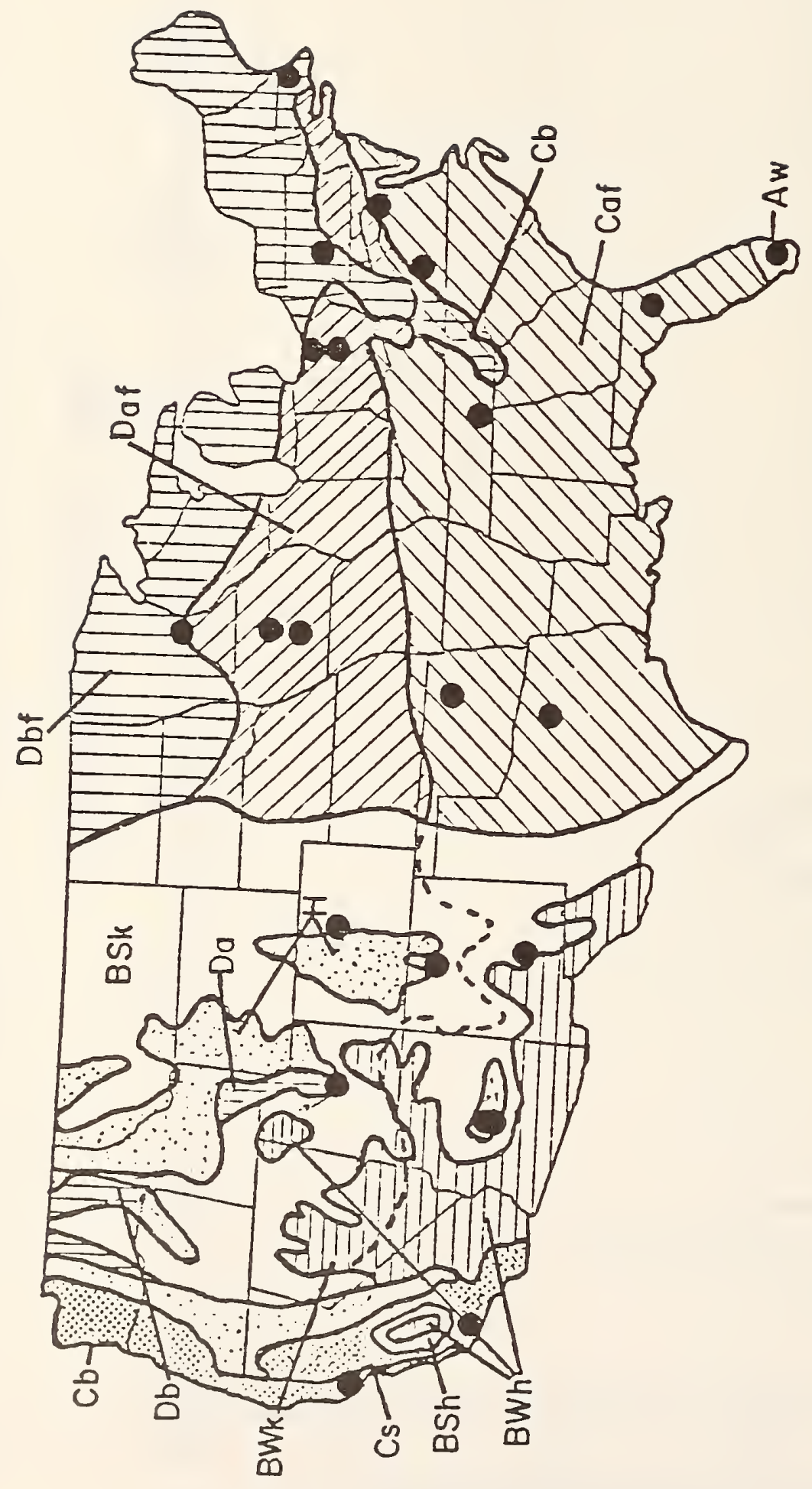

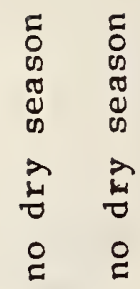

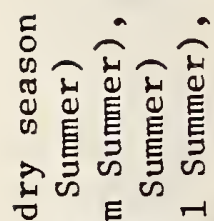

ह E

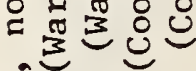

म

क

ชั

$\circlearrowleft$ व ट ख

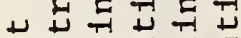

W

ऊक ठठ

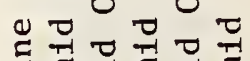

U1

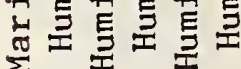

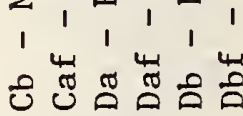

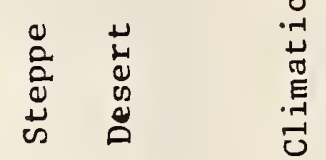

तु तु

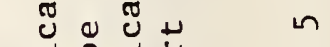

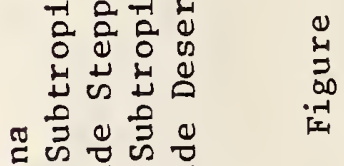

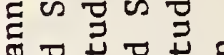

จ छ

ल

๘

यन्न न्न 가

경 응 뭉유 훙 낼

E

11112

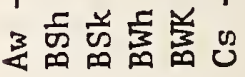




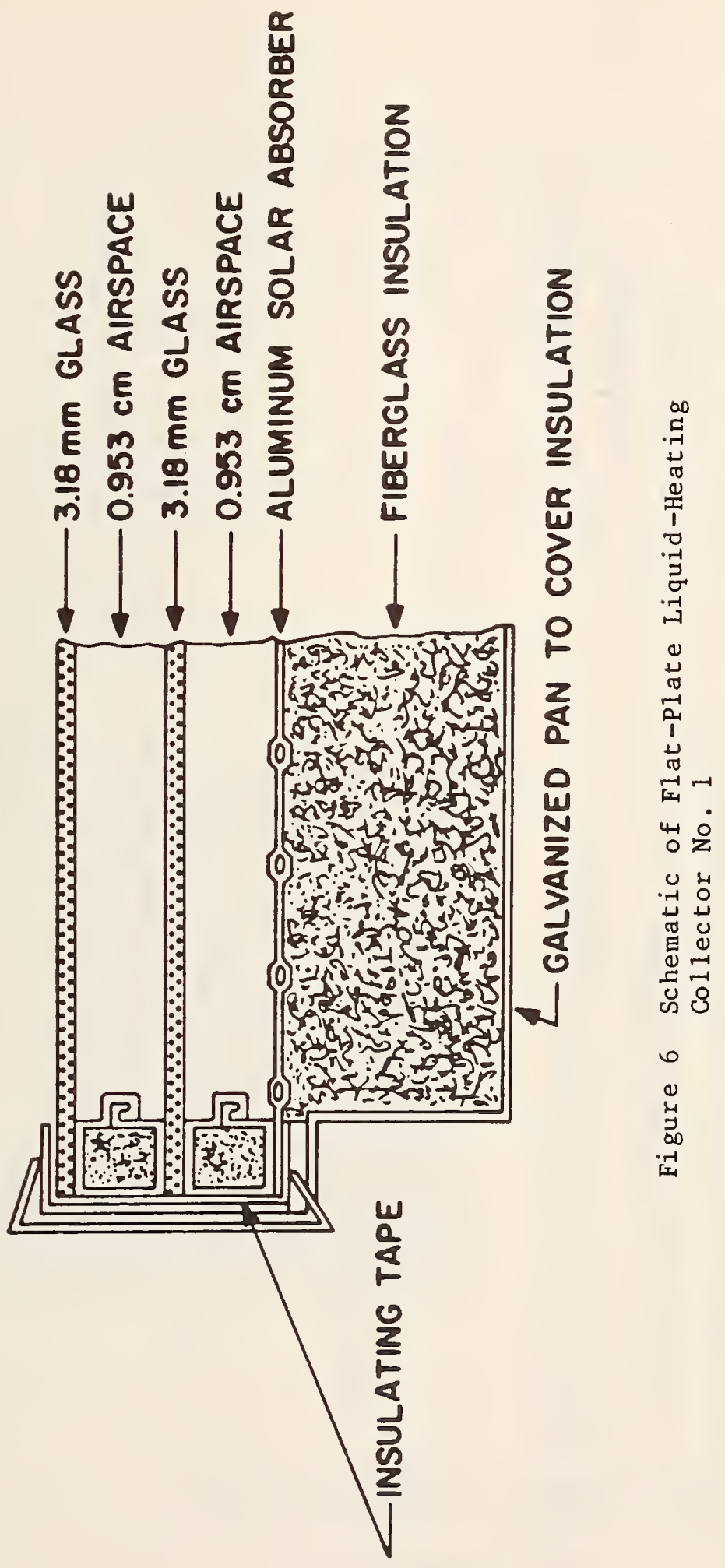




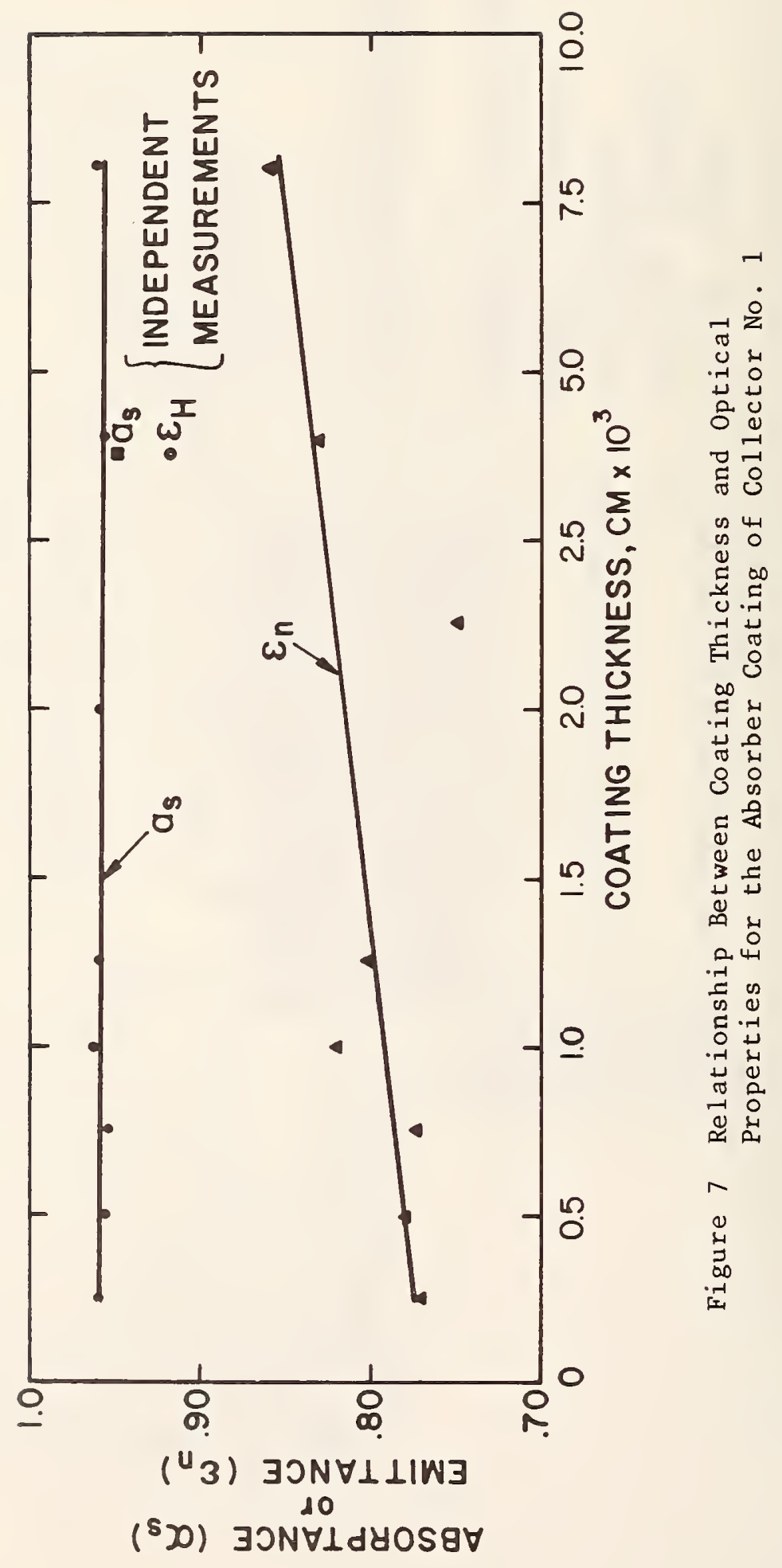




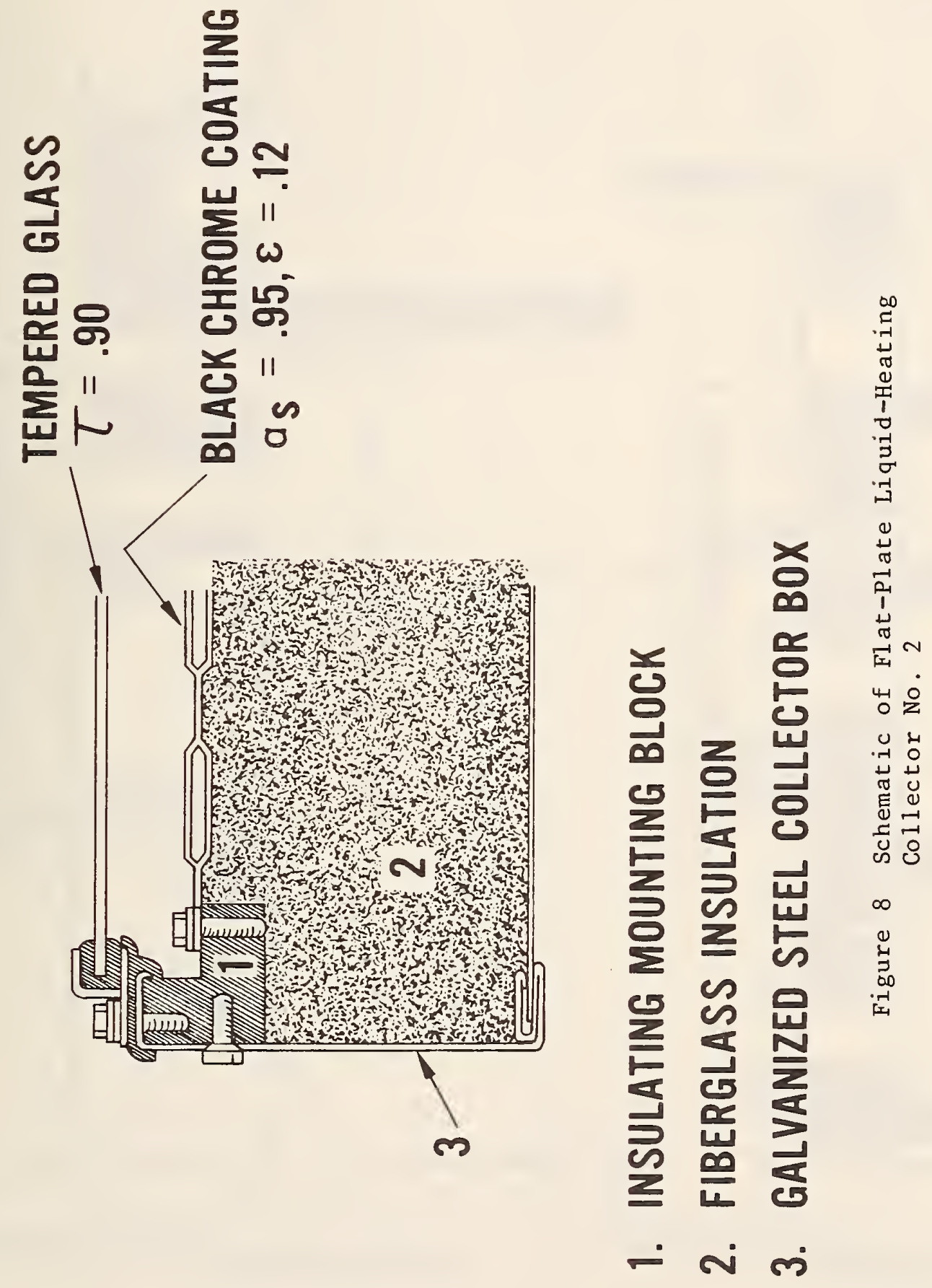




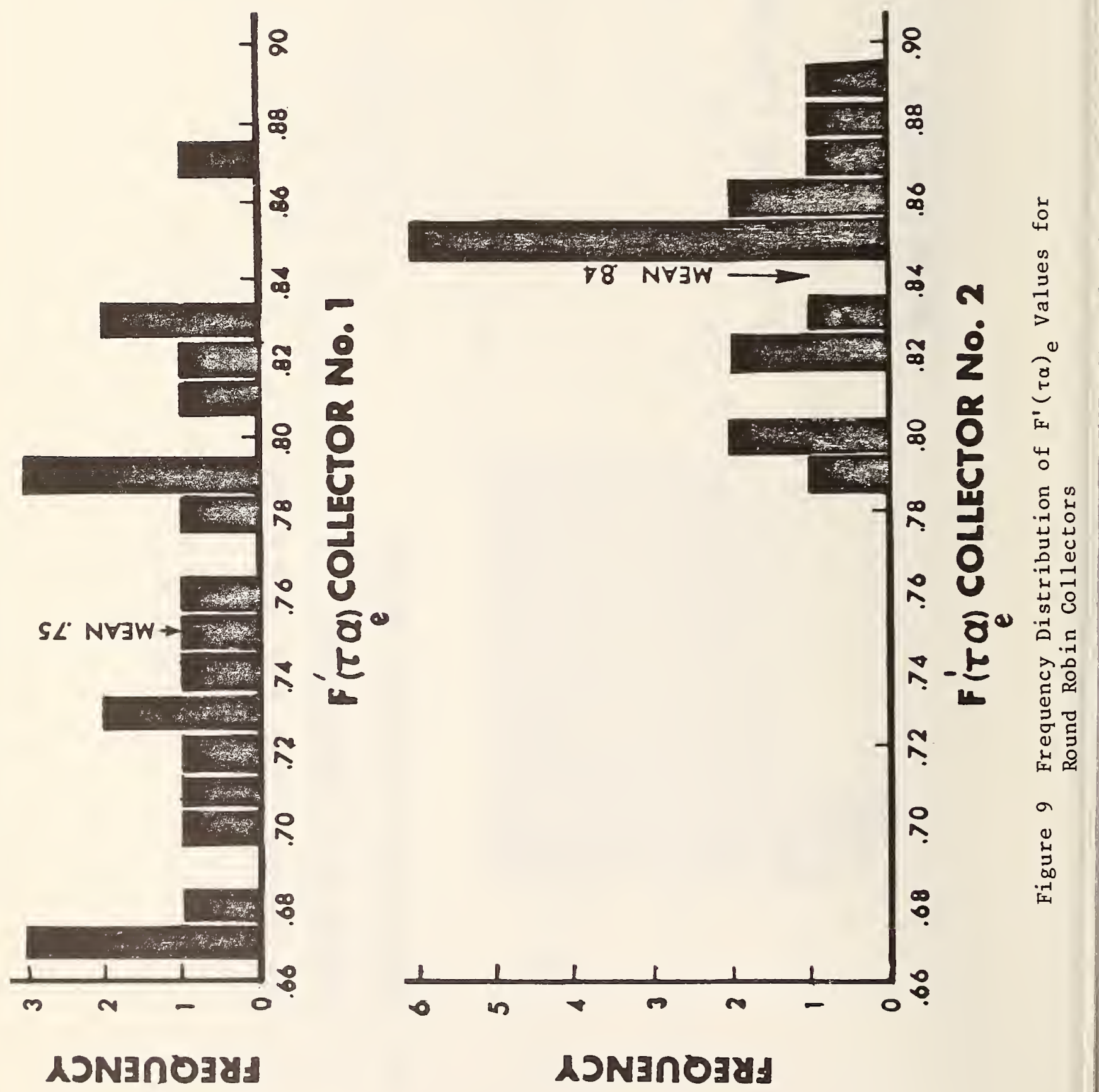



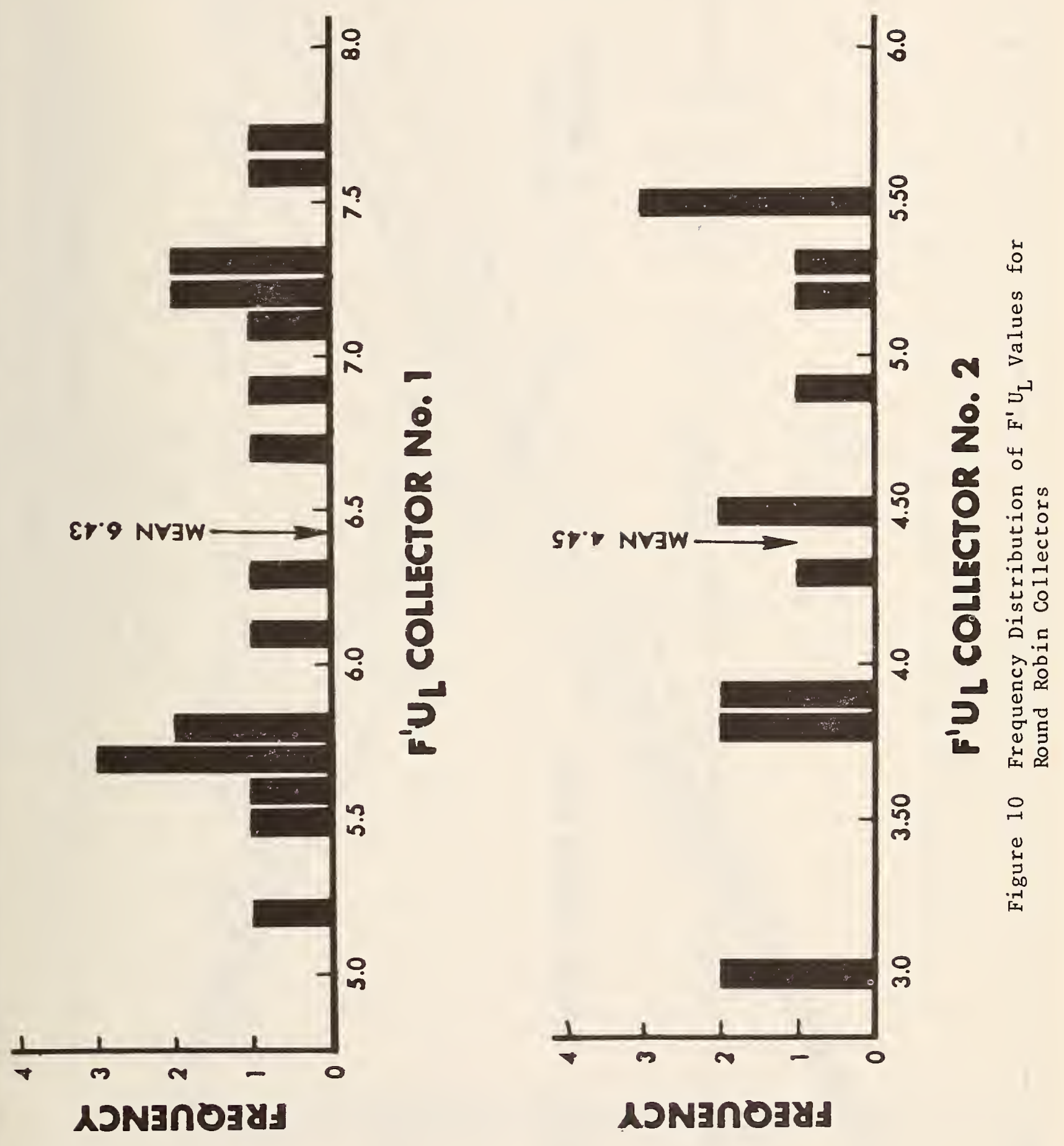


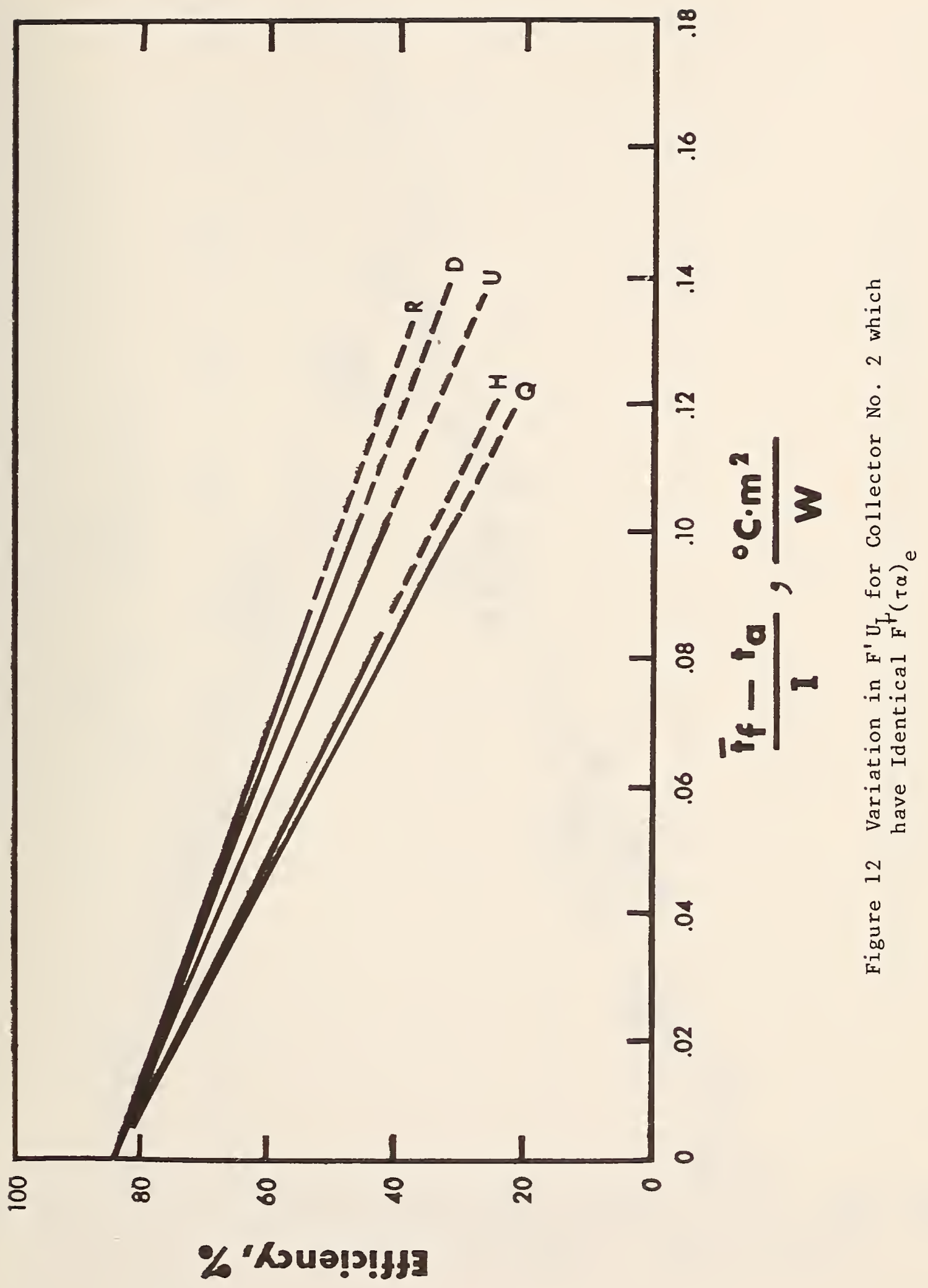




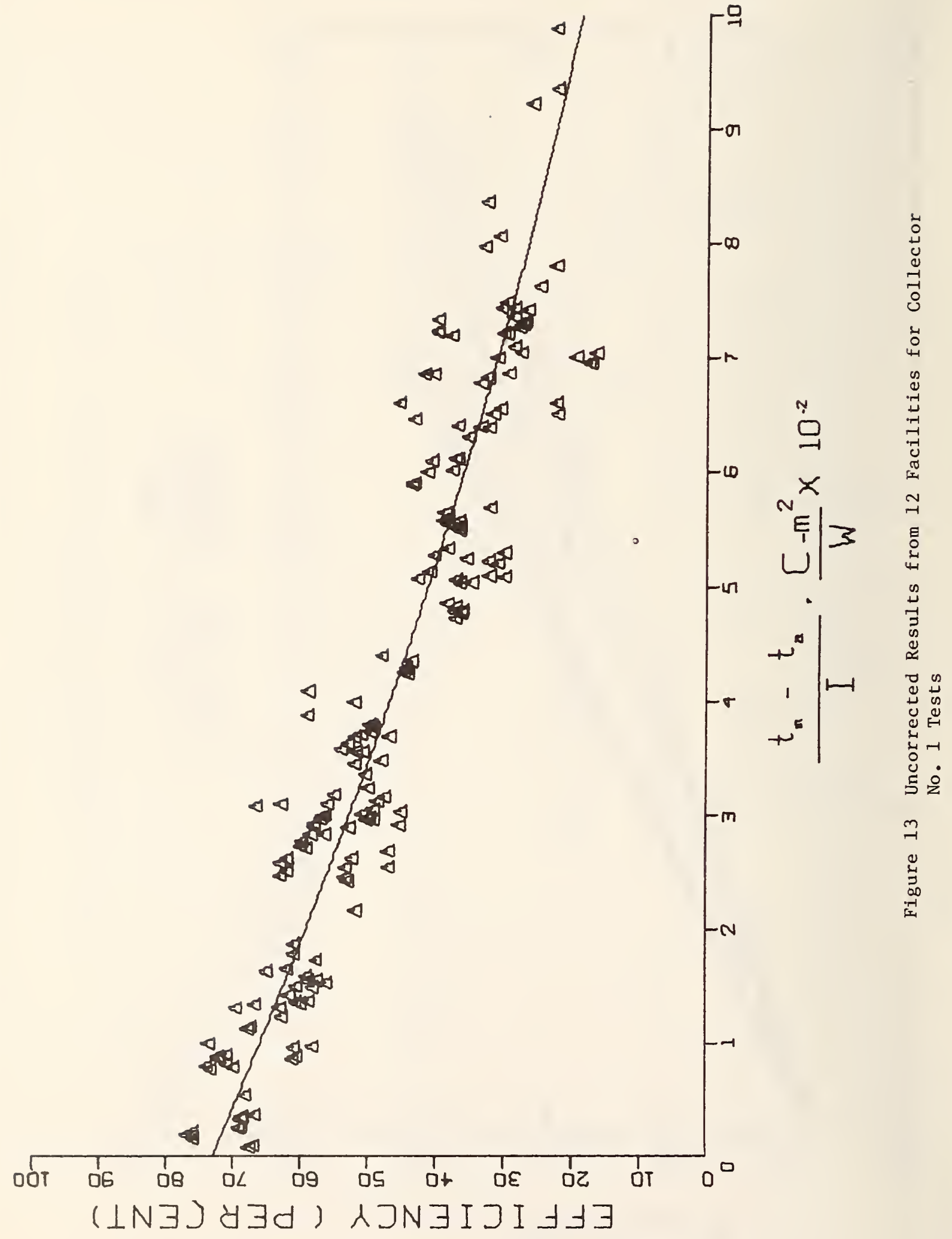




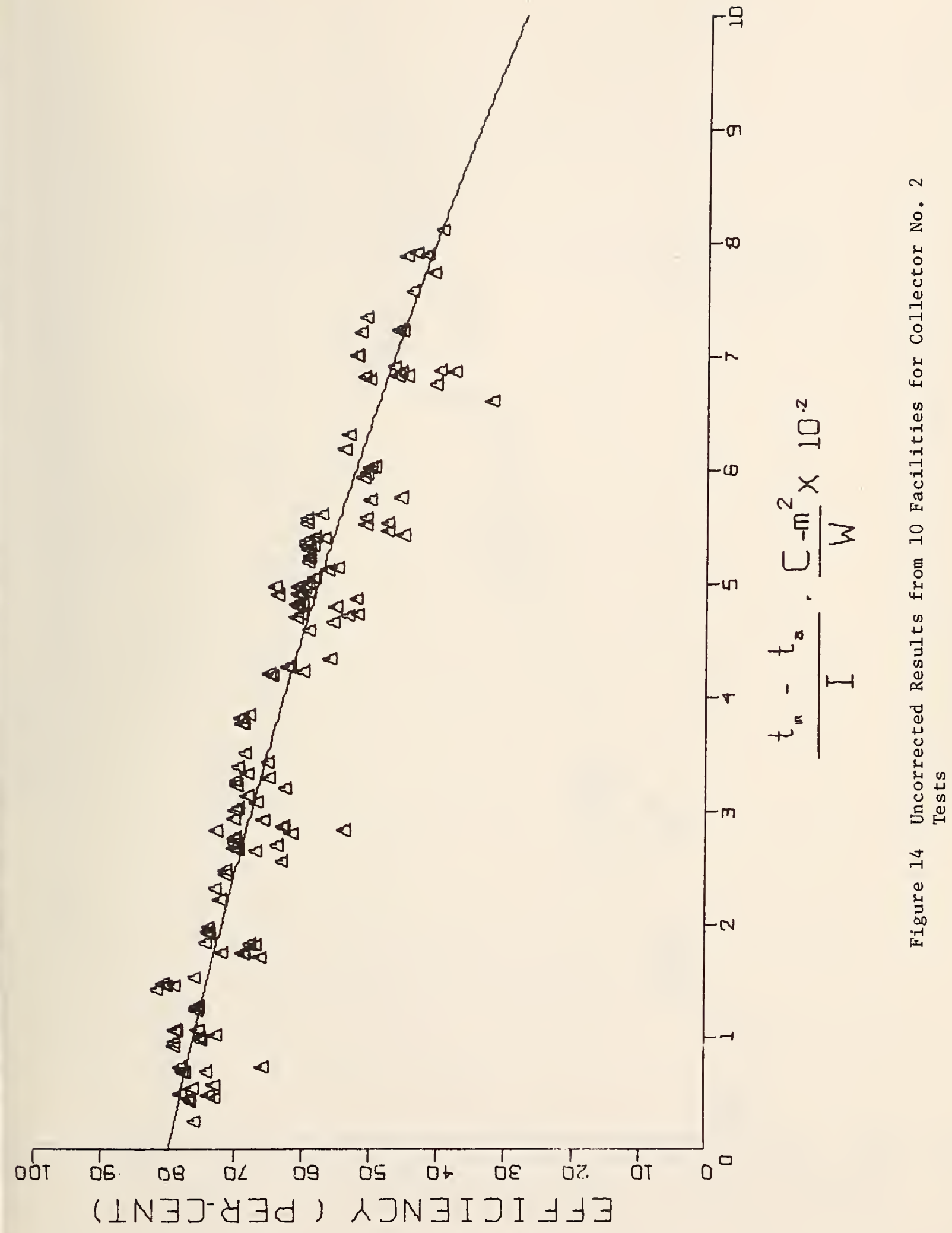




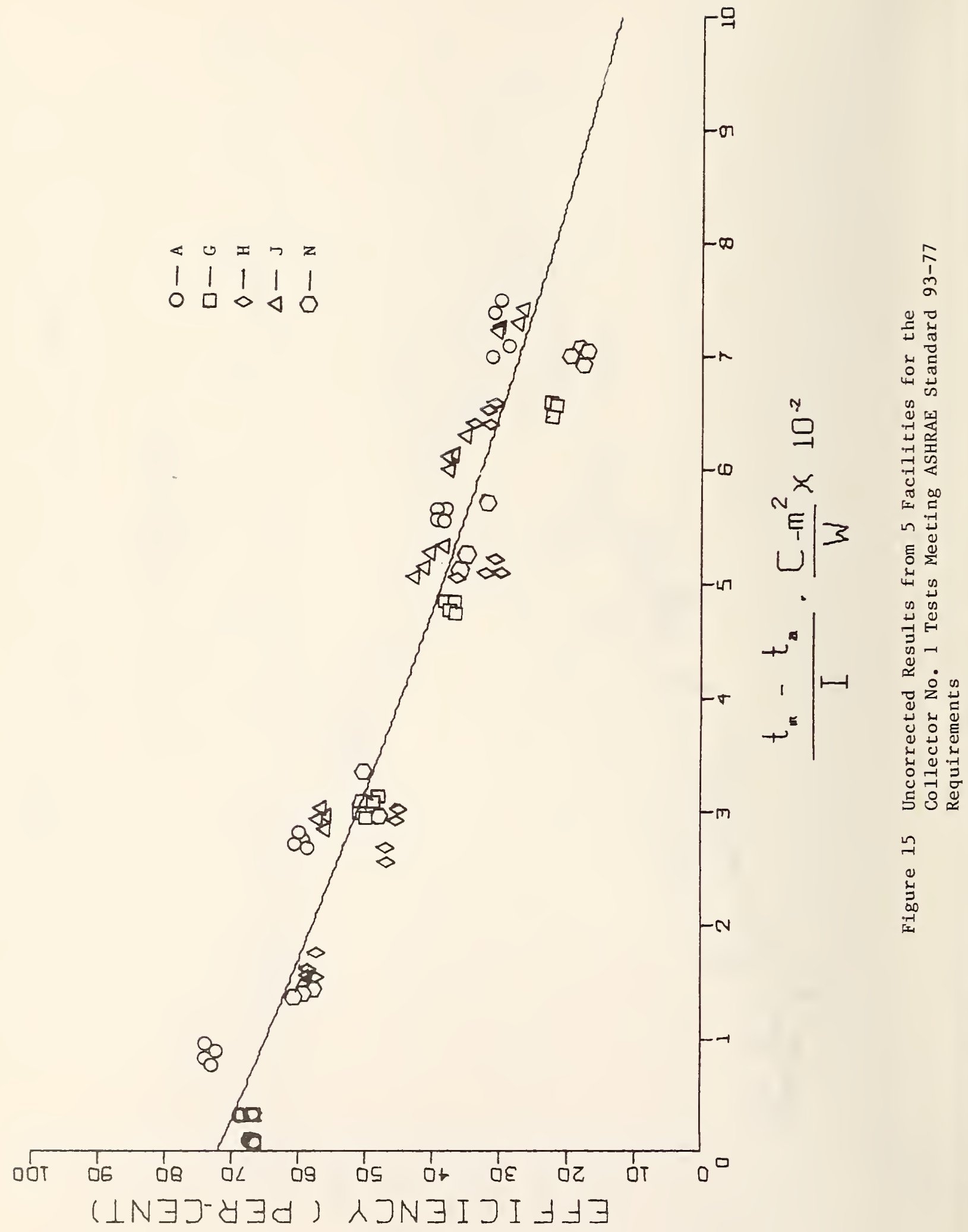




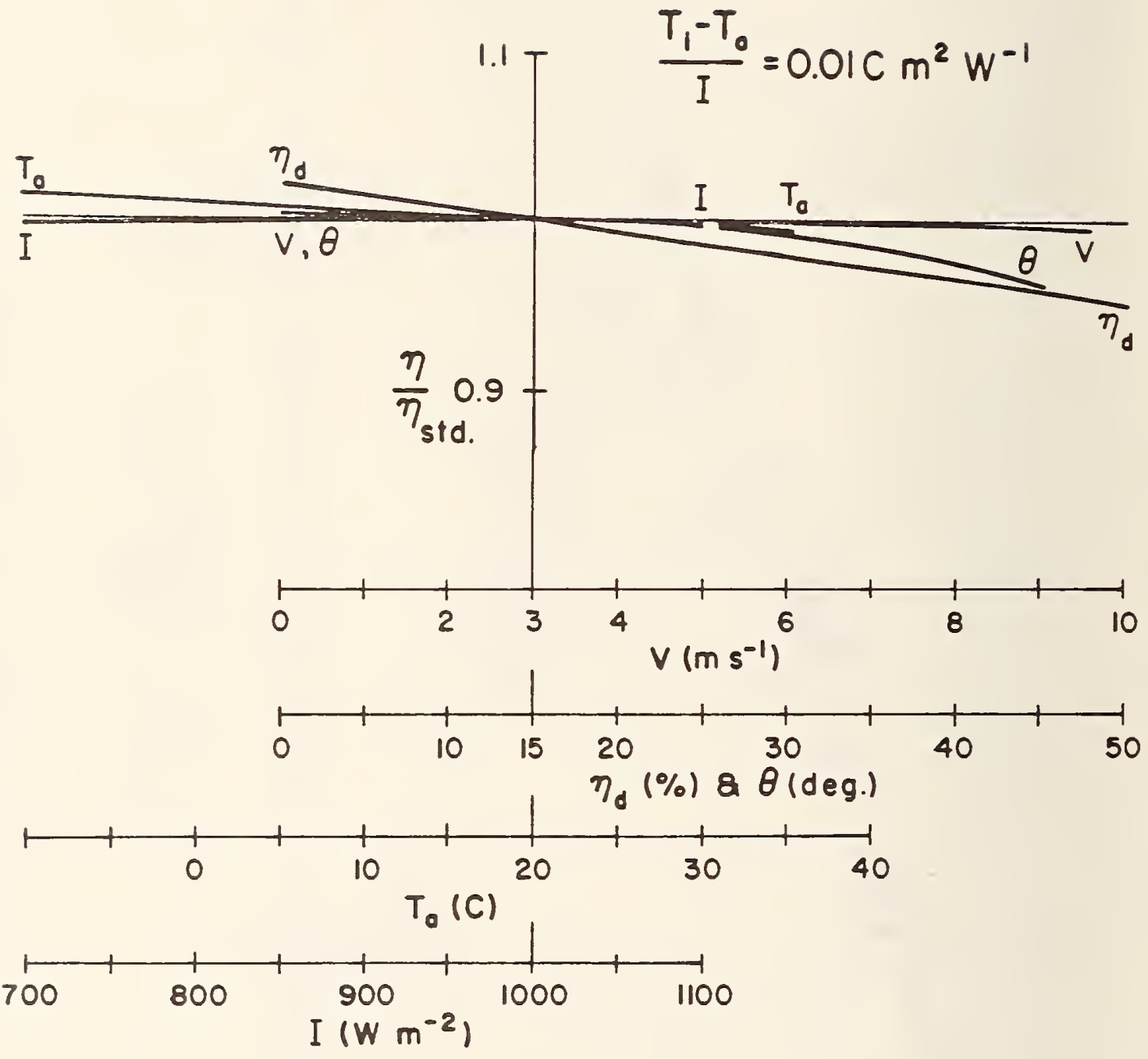

Figure 17 Effect of Environmental Conditions on Efficiency for Collector No. 1 at "Reference" Conditions with $\left[t_{f, i}-t_{a}\right] / I=0.01\left({ }^{\circ} \mathrm{C} \cdot \mathrm{m}^{2}\right) / \mathrm{W}$ 


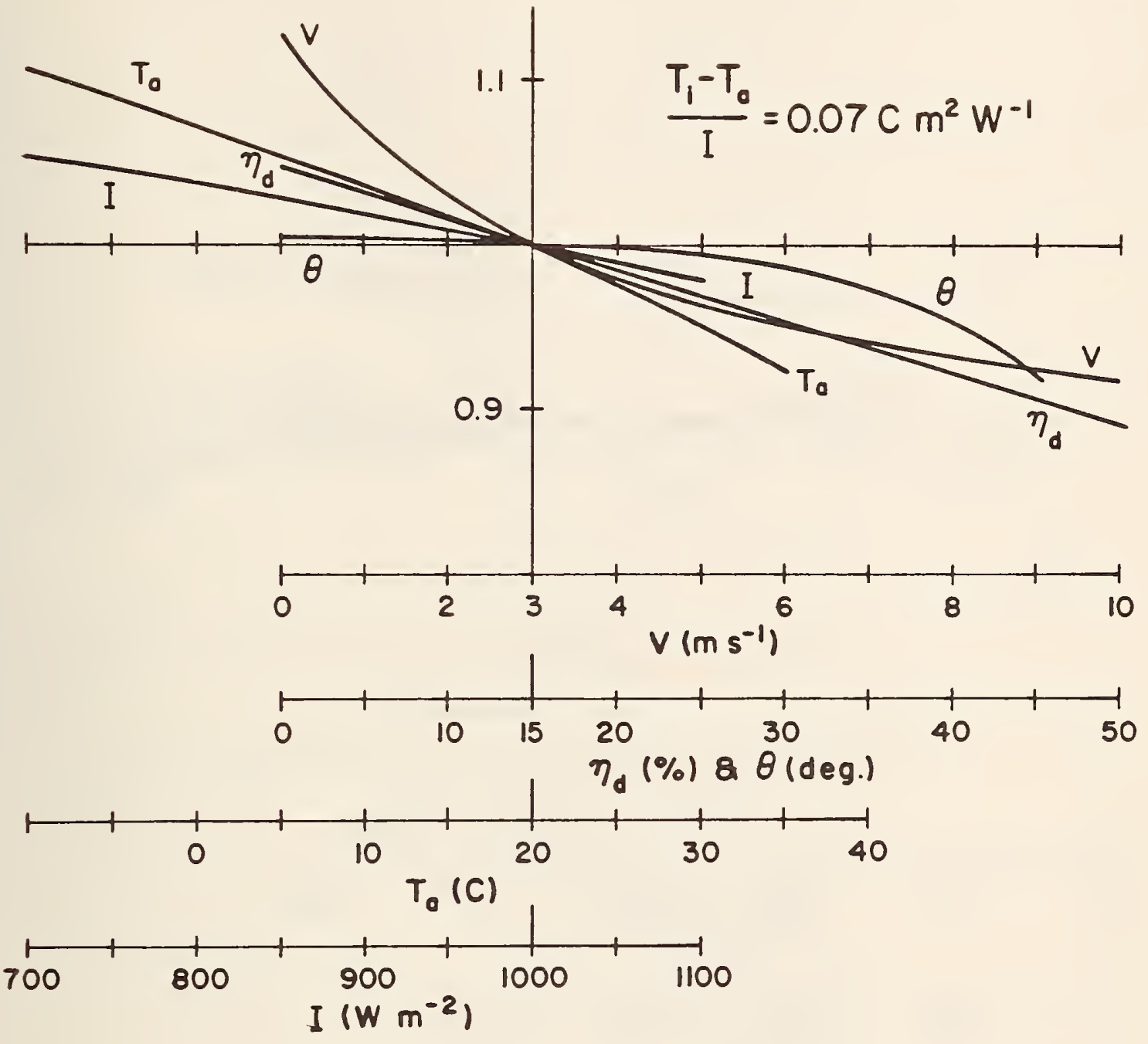

Figure 18 Effect of Environmental Conditions on Efficiency for Collector No. 1 at "Reference" Conditions with $\left[t_{f, i}-t_{a}\right] / I=0.07\left({ }^{\circ} \mathrm{C} \cdot \mathrm{m}^{2}\right) / \mathrm{W}$ 


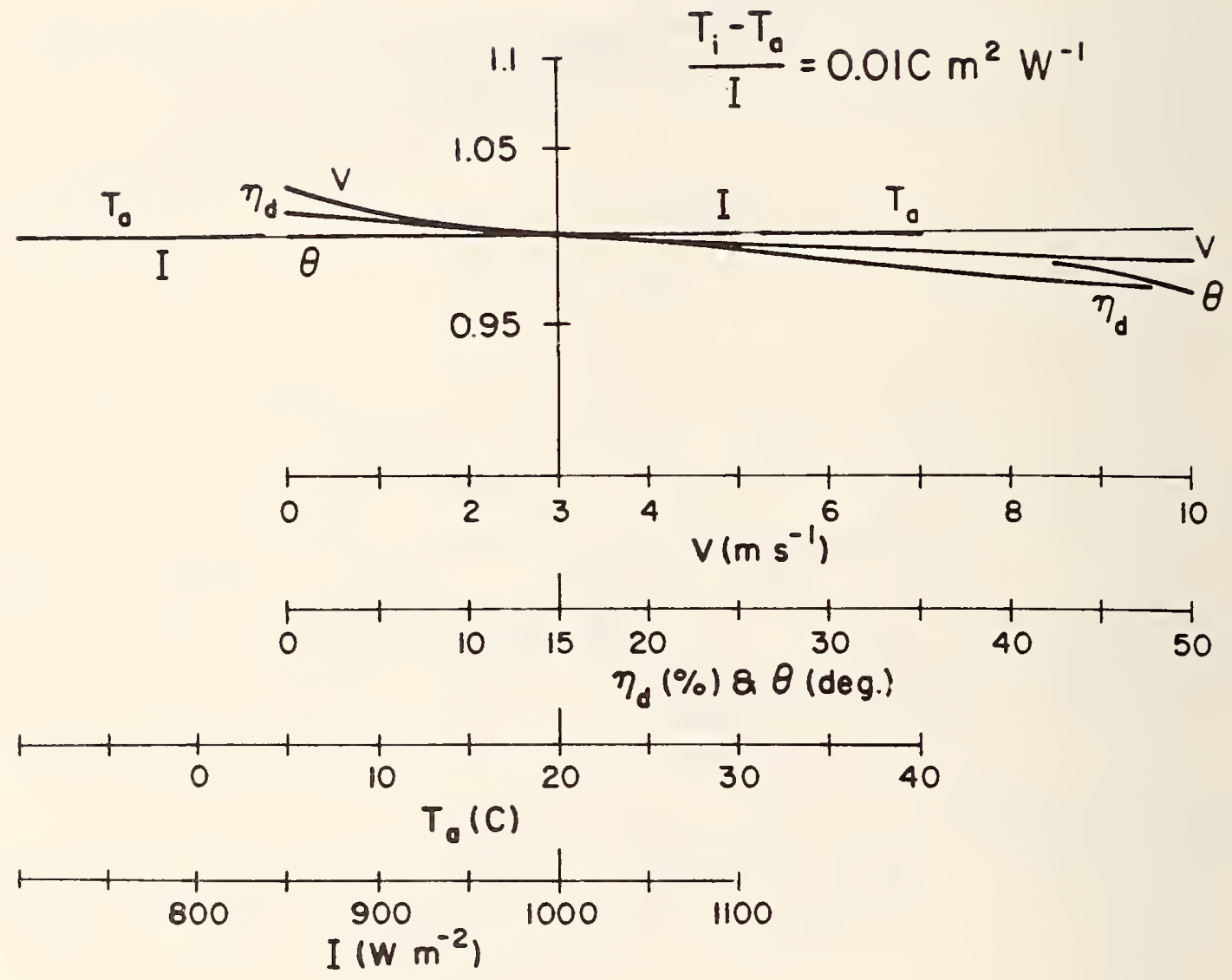

Figure 19 Effect of Environmental Conditions on Efficiency for Collector No. 2 at "Reference" Conditions with $\left[\mathrm{t}_{\mathrm{f}, \mathrm{i}}-\mathrm{t}_{\mathrm{a}}\right] / \mathrm{I}=0.01\left({ }^{\circ} \mathrm{C} \cdot \mathrm{m}^{2}\right) / \mathrm{W}$ 


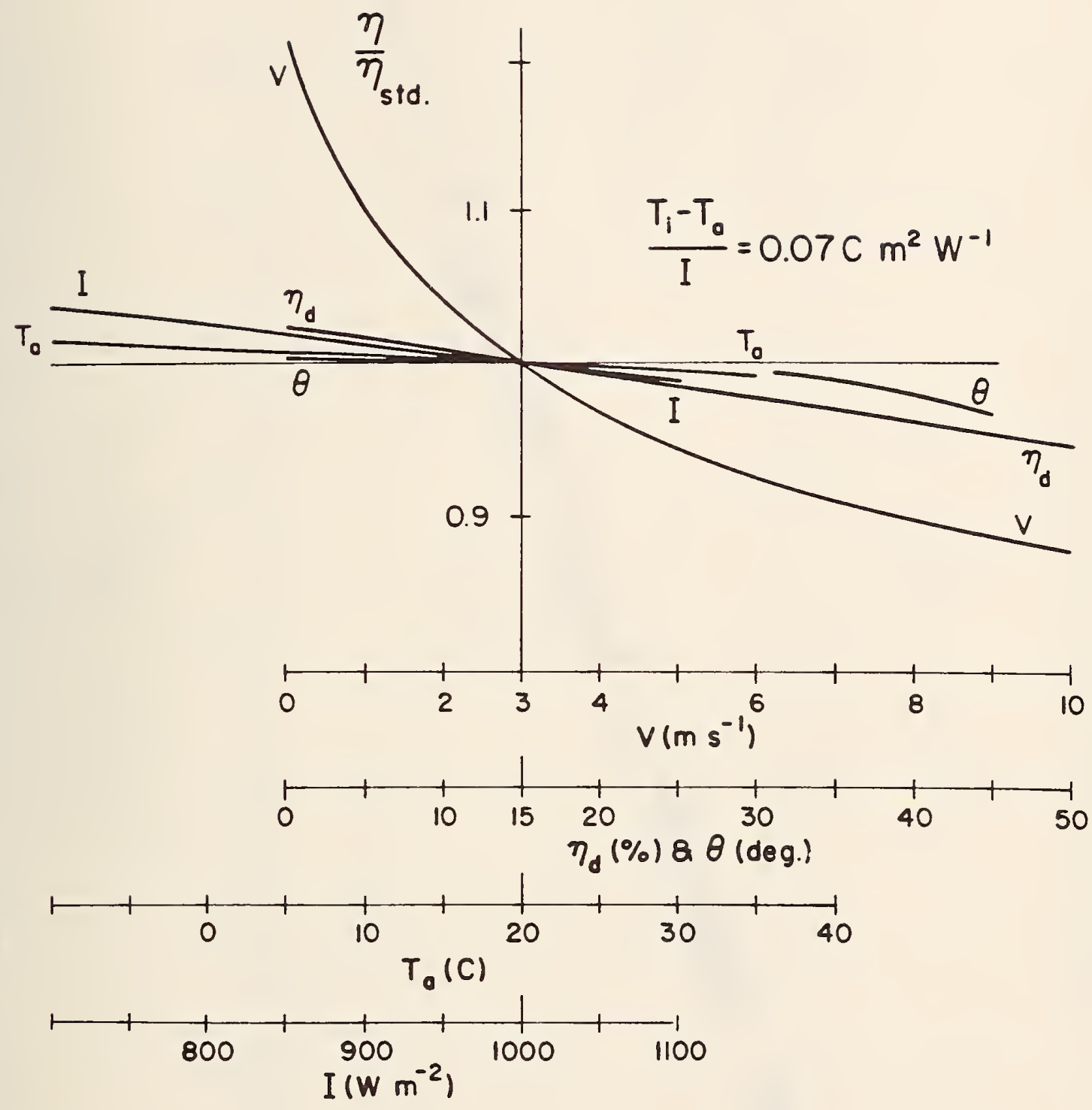

Figure 20 Effect of Environmental Conditions on Efficiency for Collector No. 2 at "Reference" Conditions with $\left[t_{f, i}-t_{a}\right] / I=0.07\left({ }^{\circ} \mathrm{C} \cdot \mathrm{m}^{2}\right) / \mathrm{W}$ 


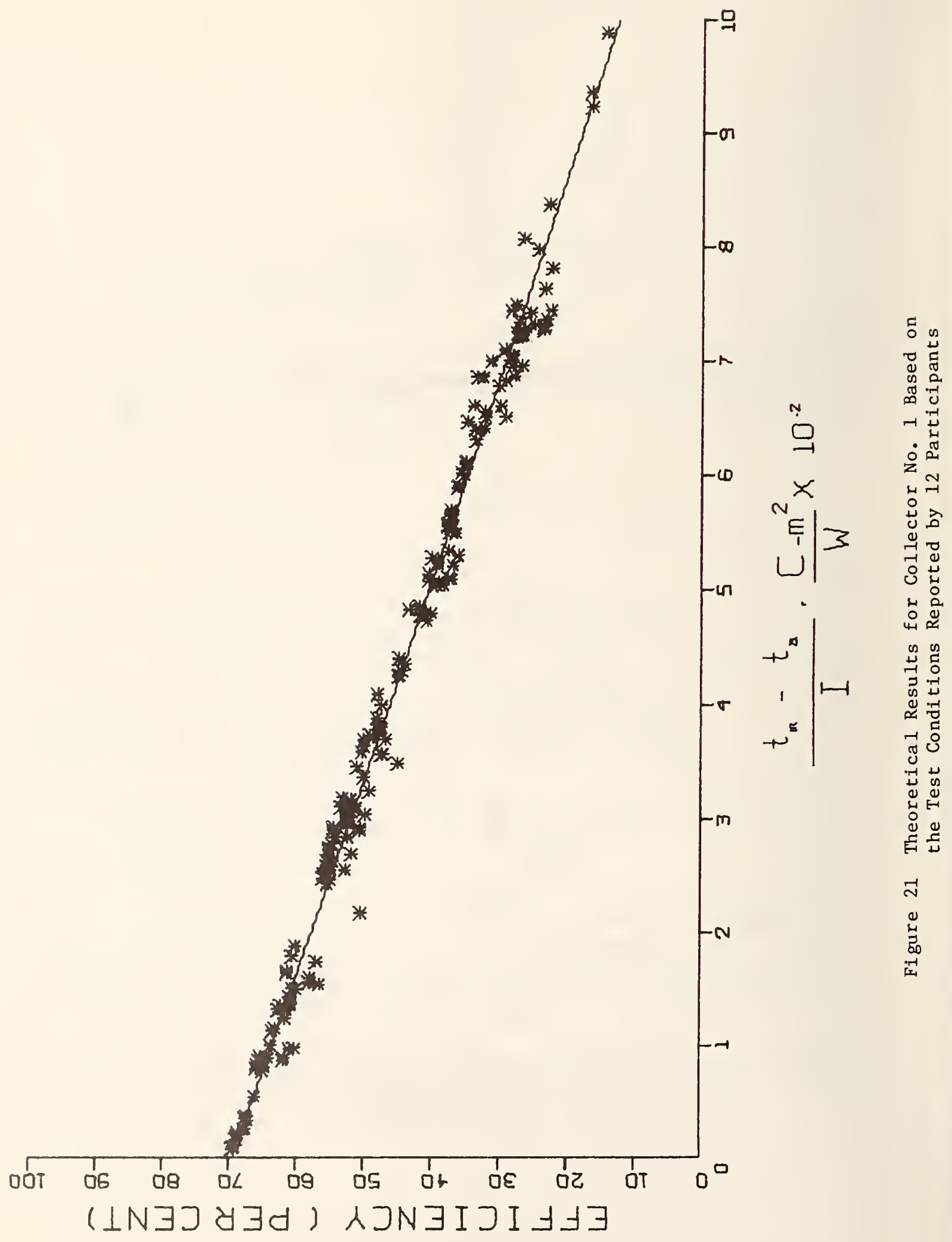




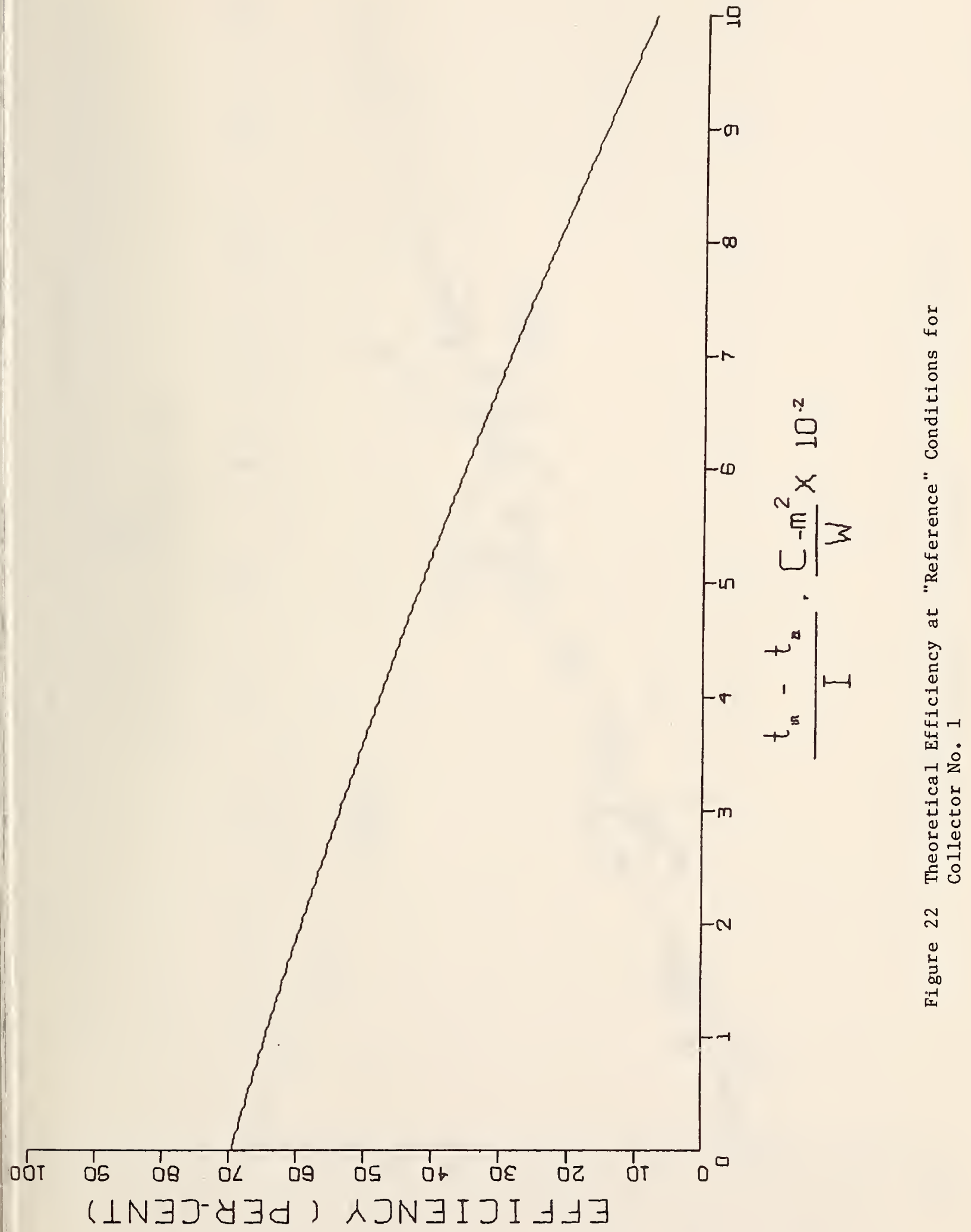




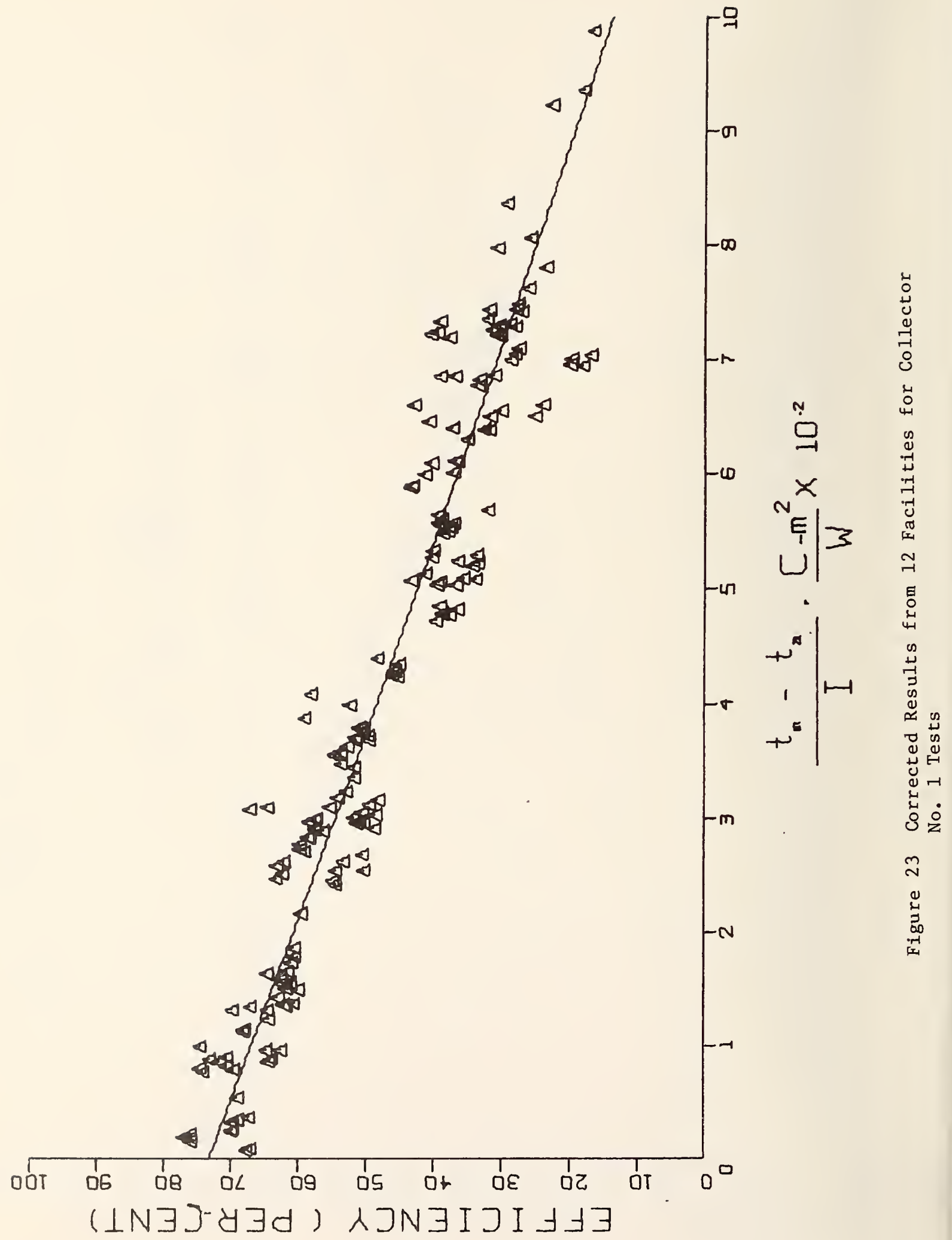




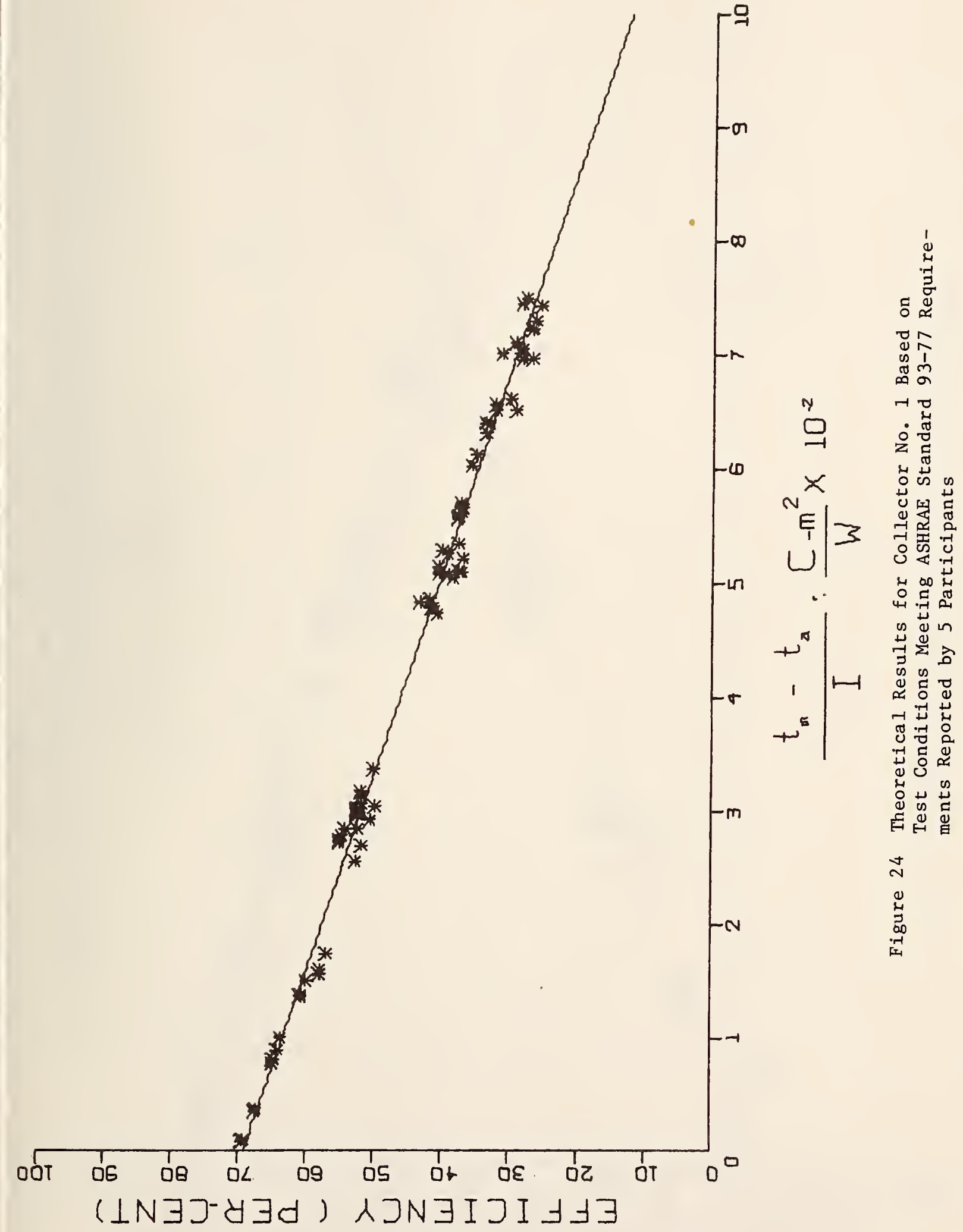




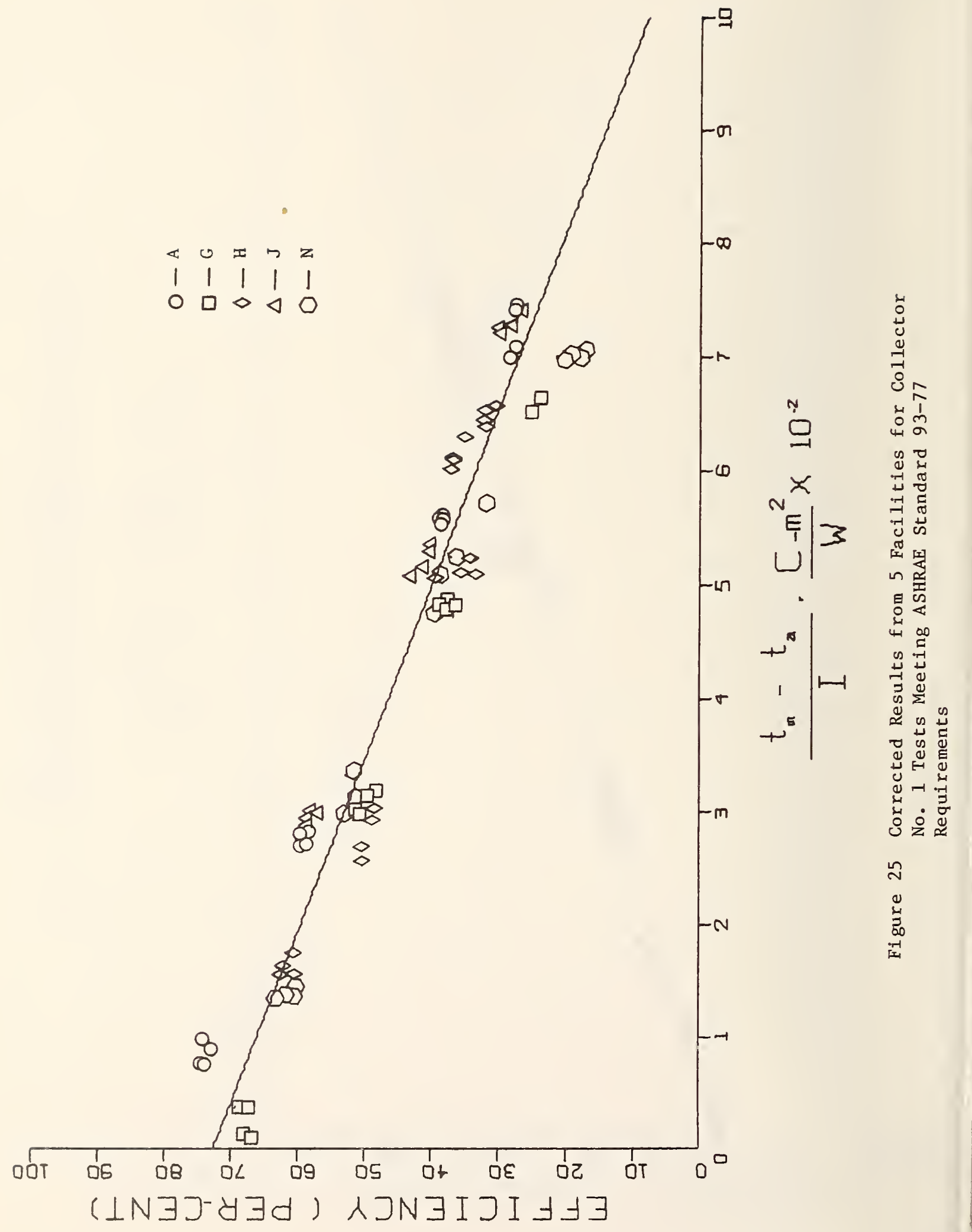




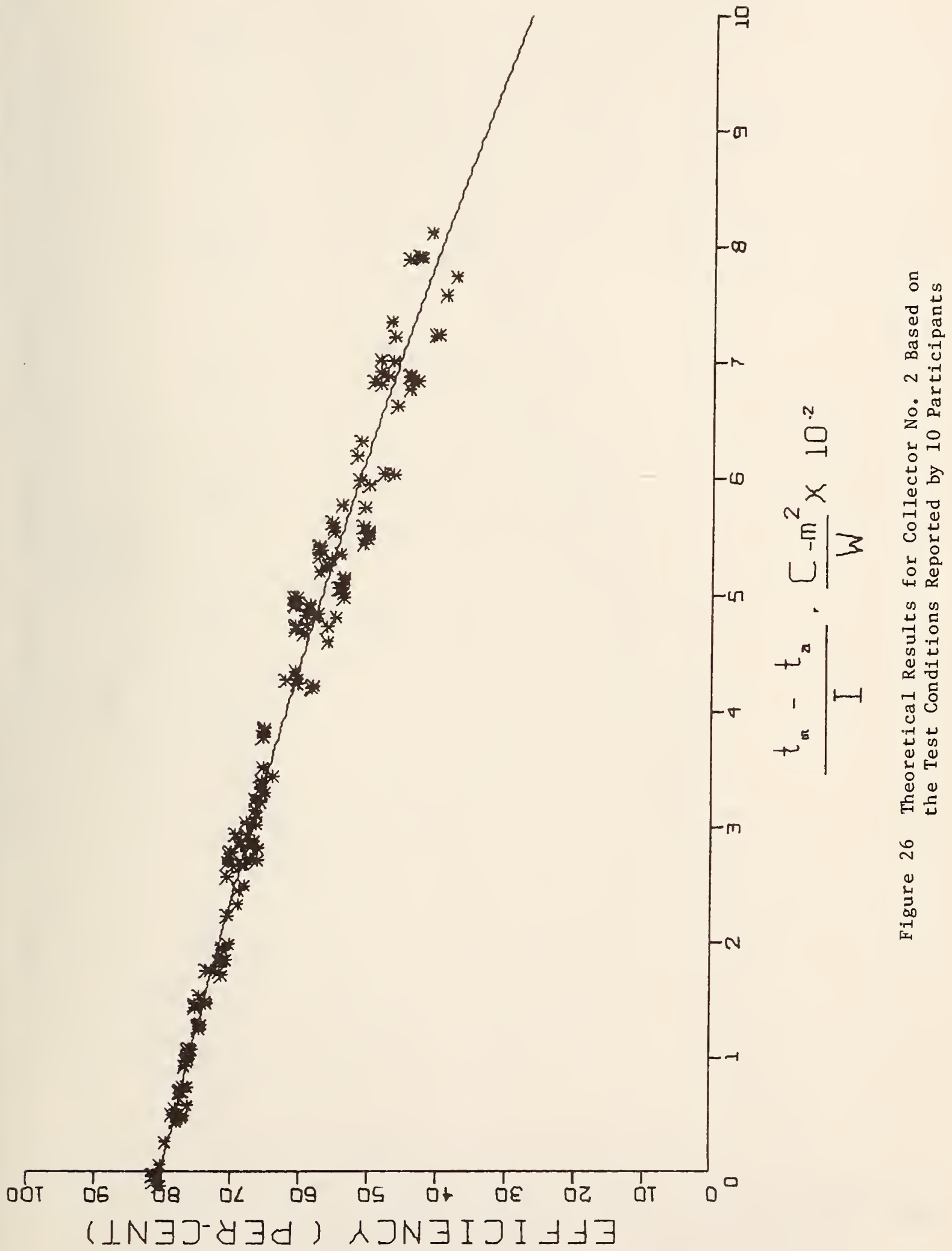




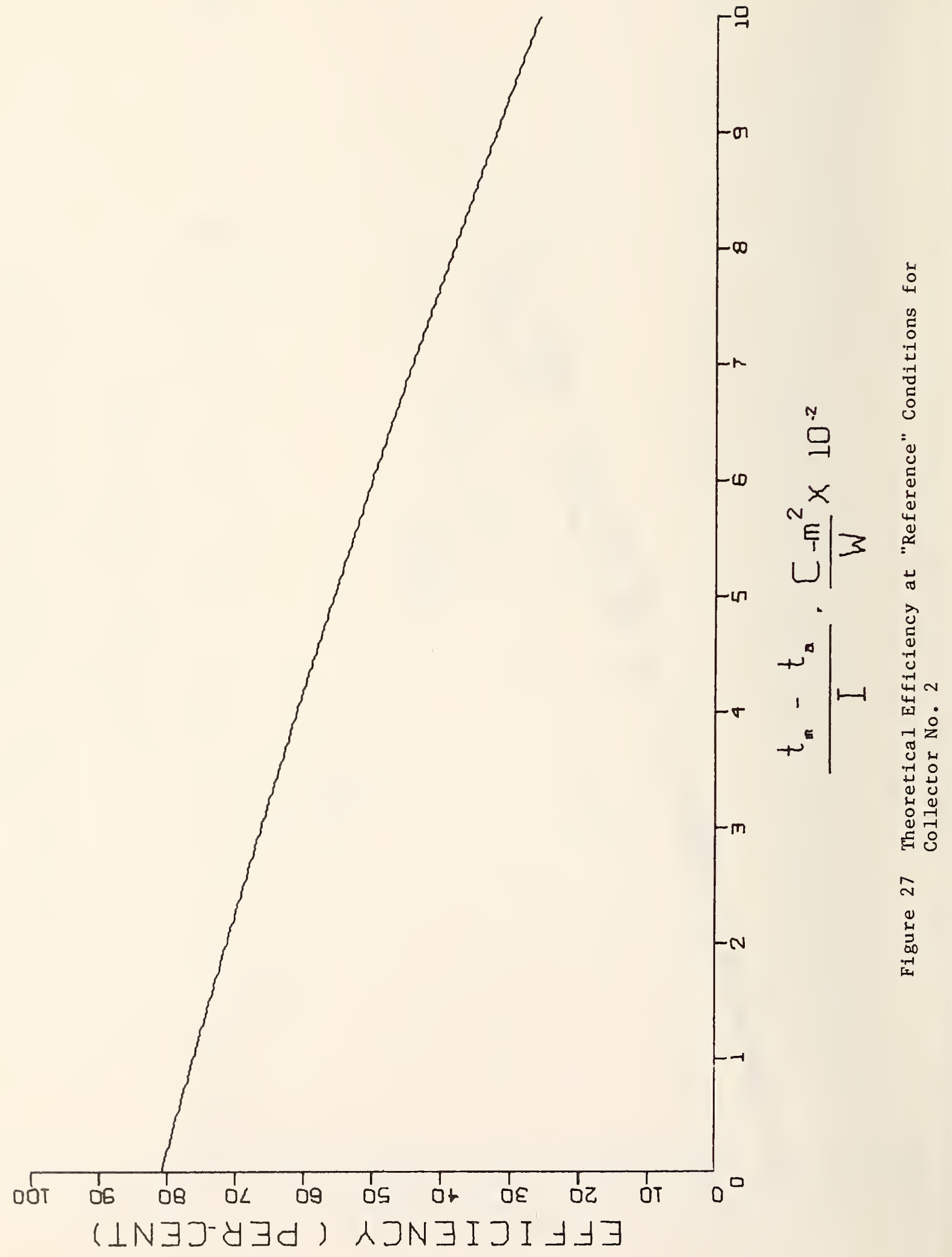




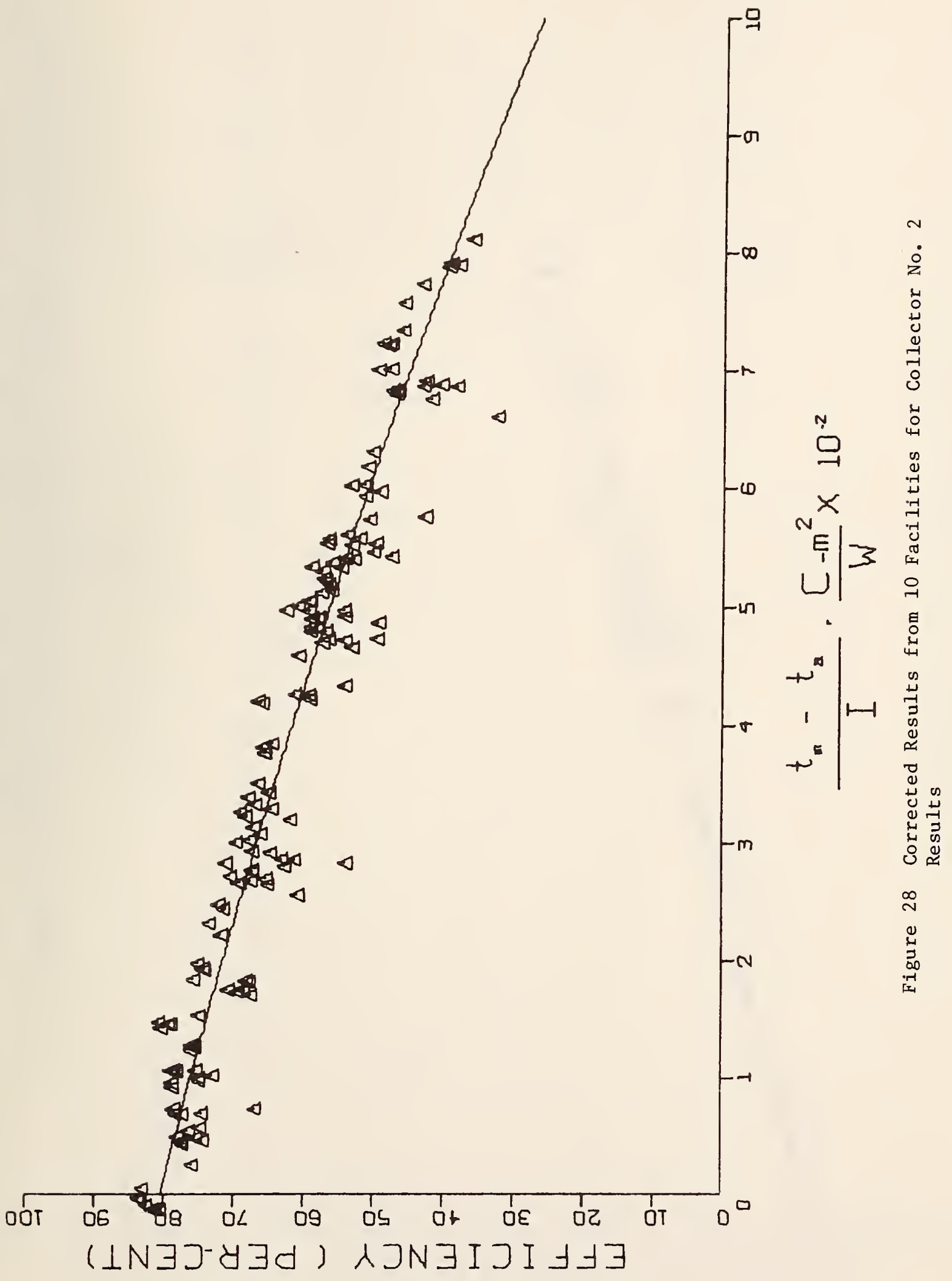




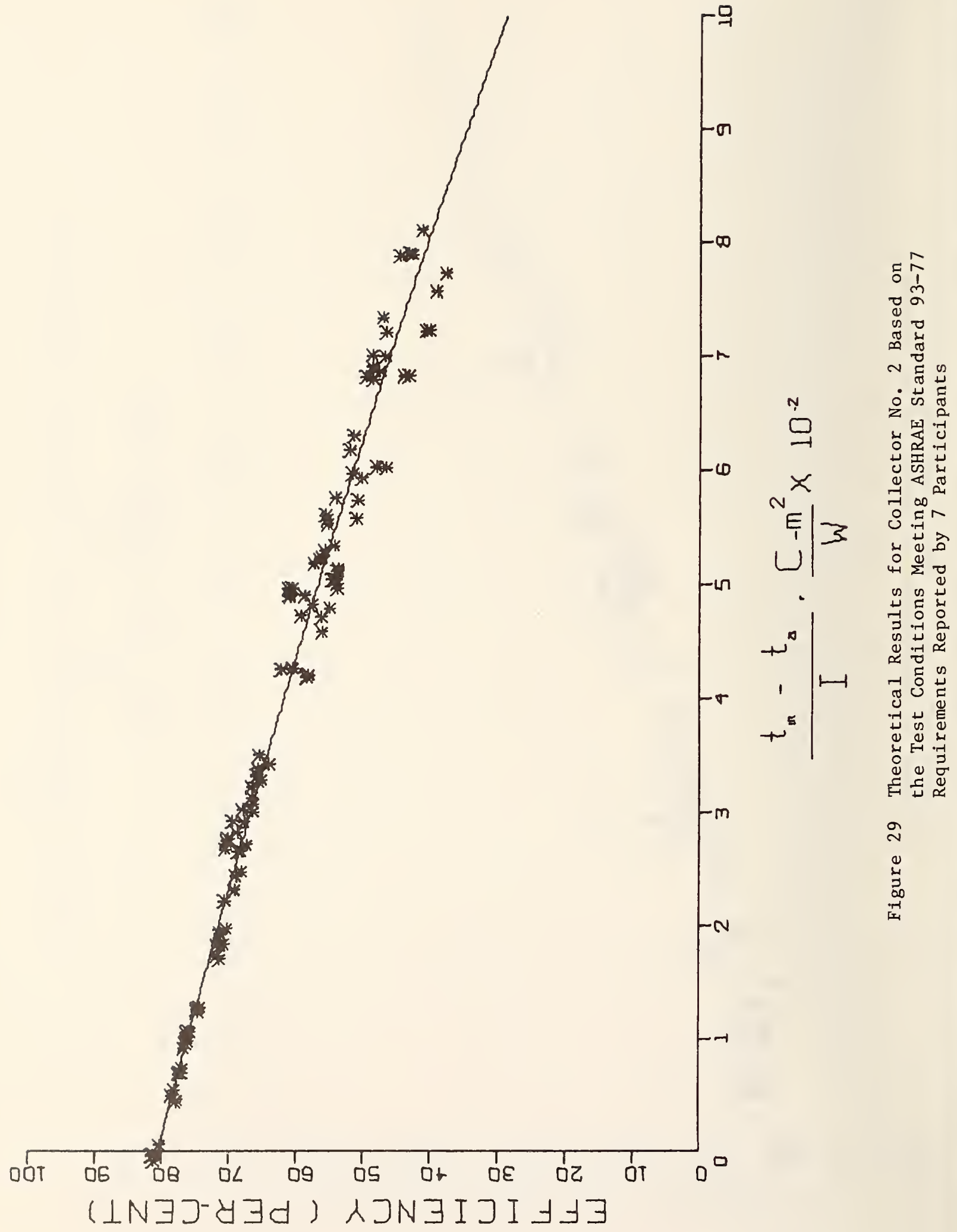




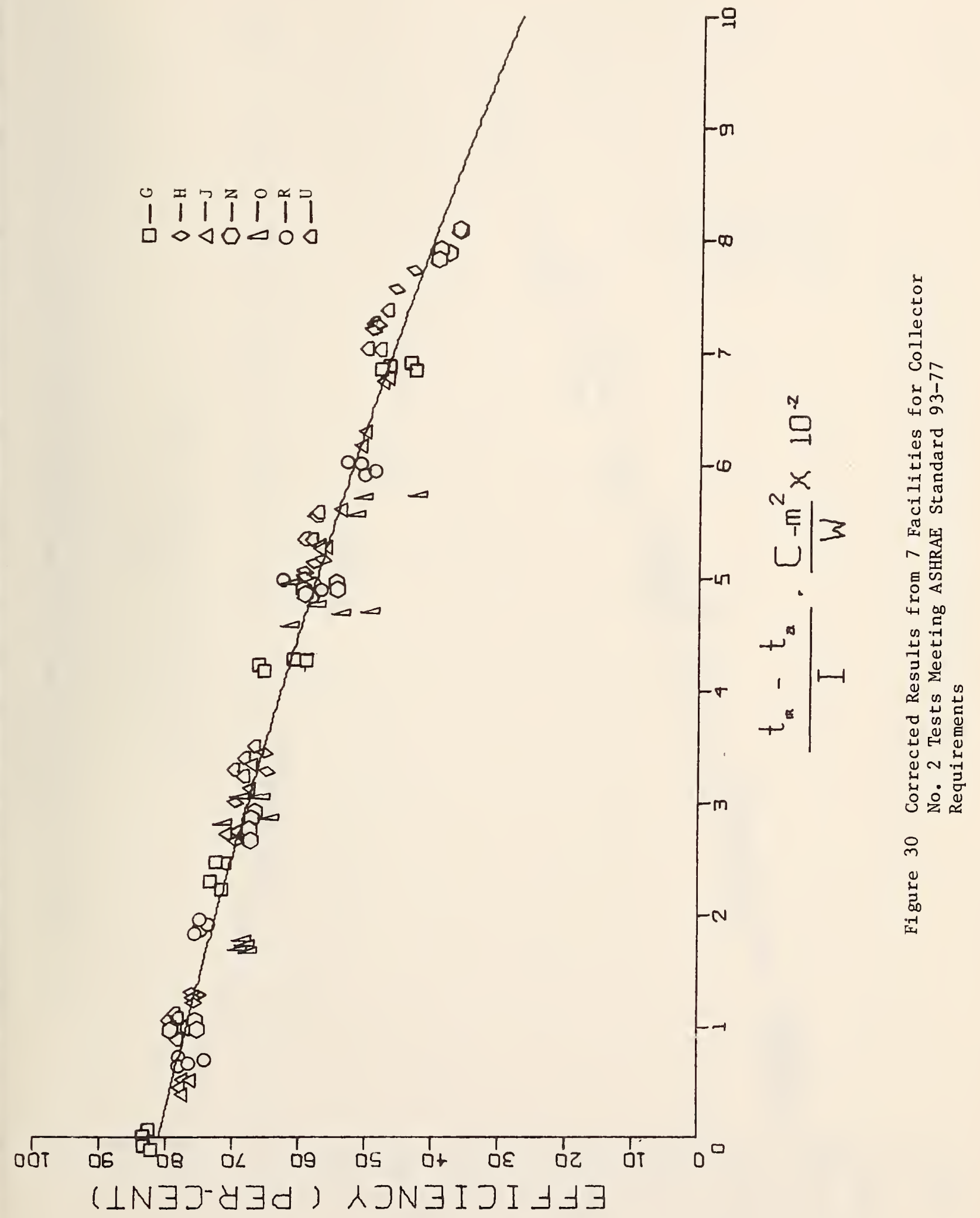




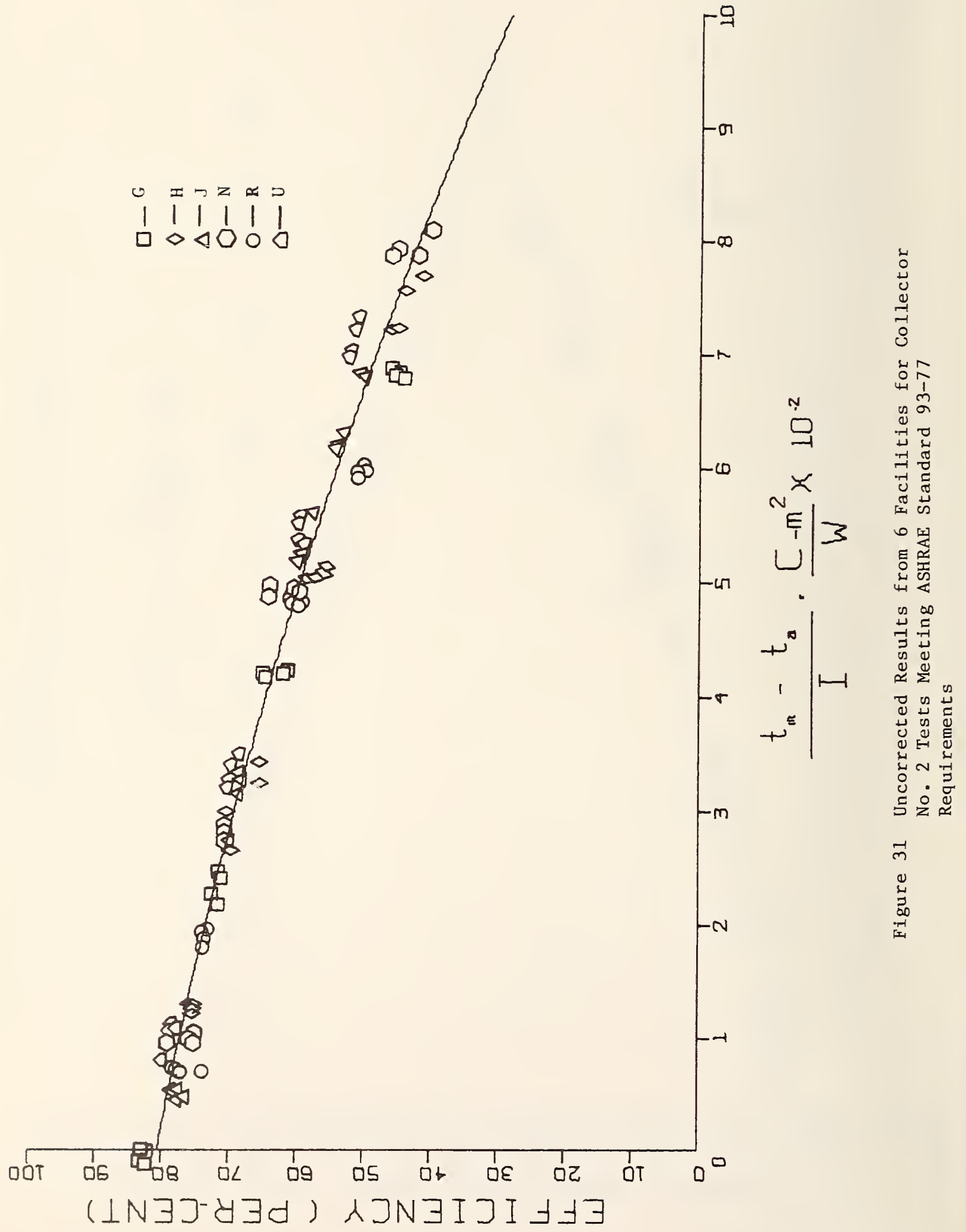




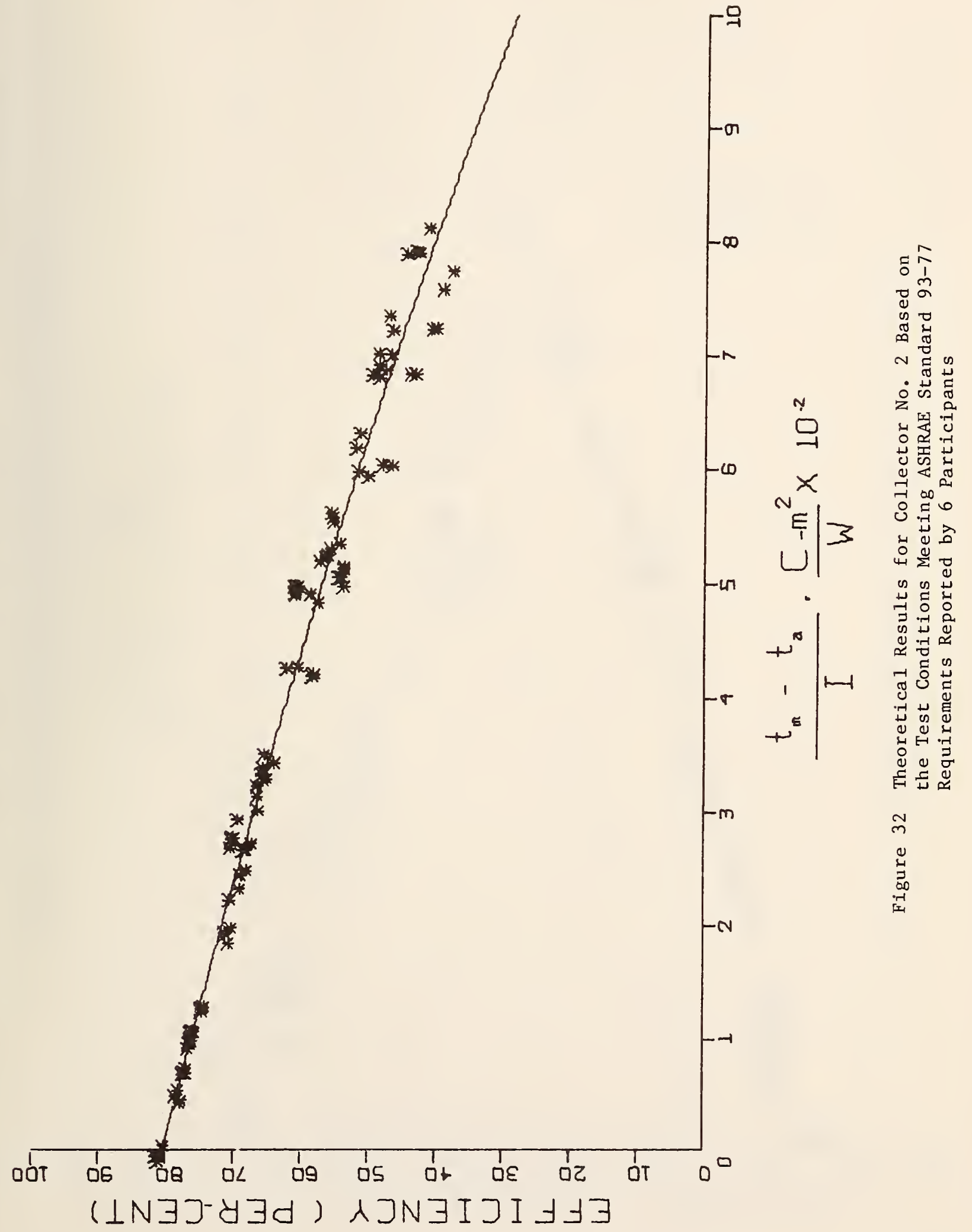




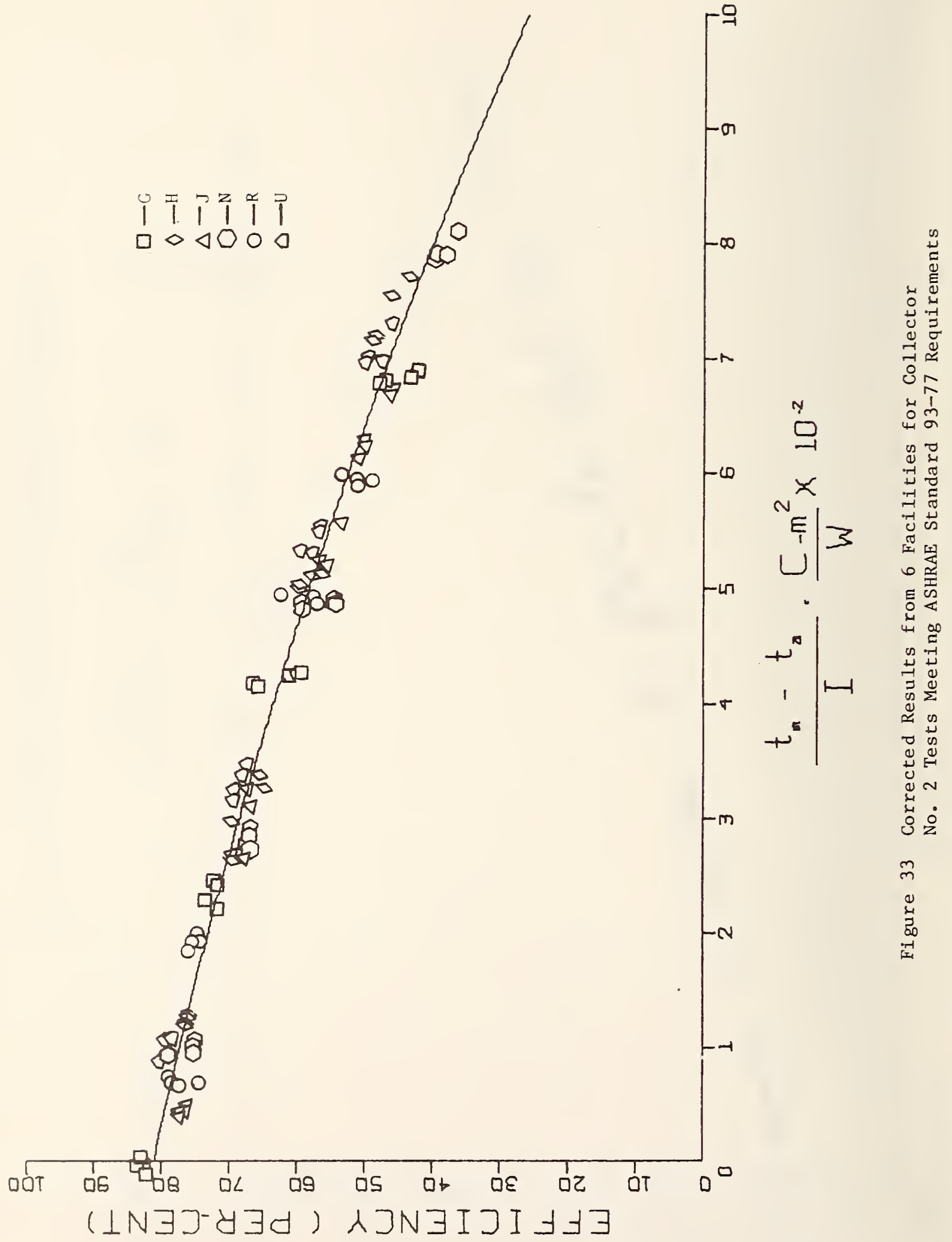




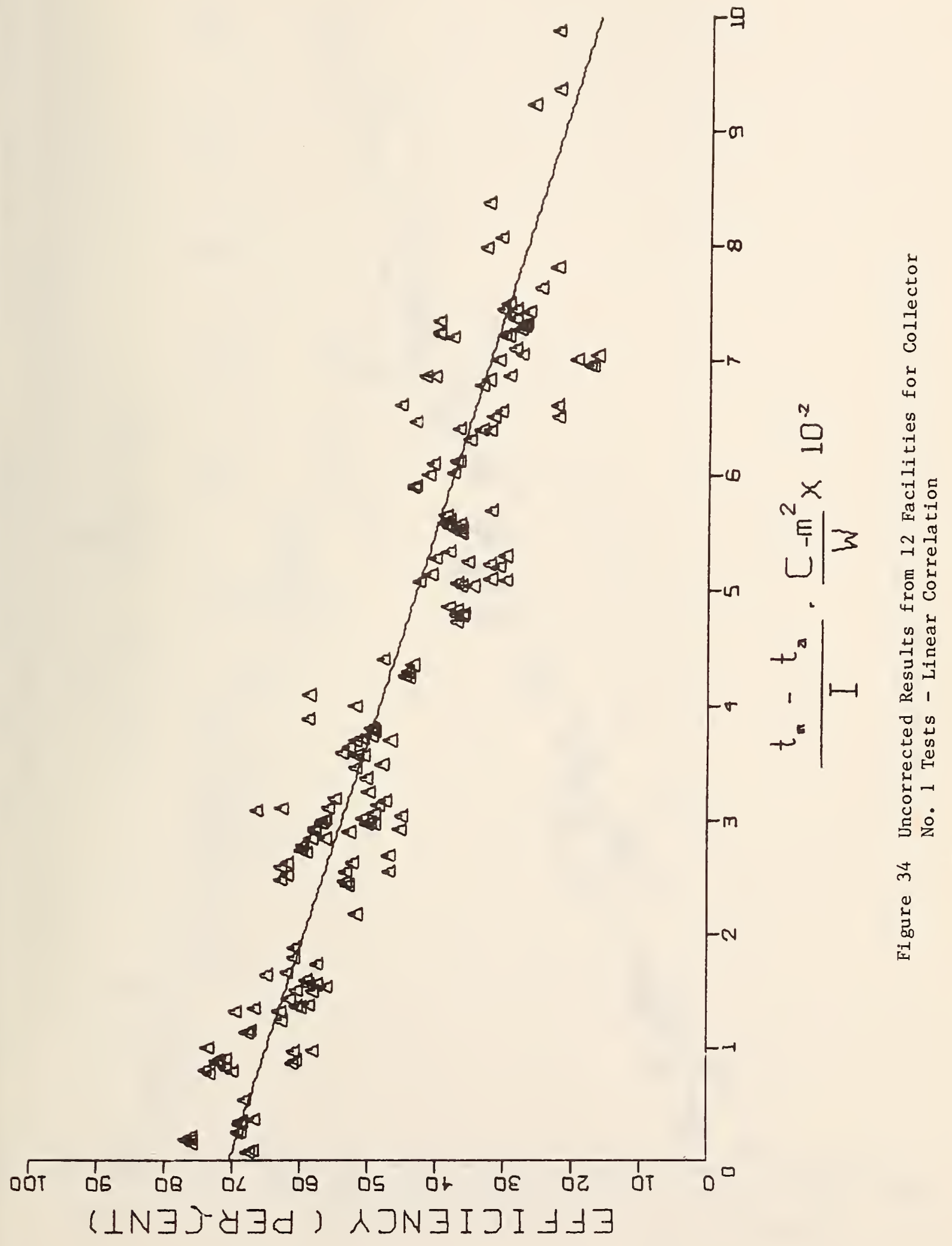




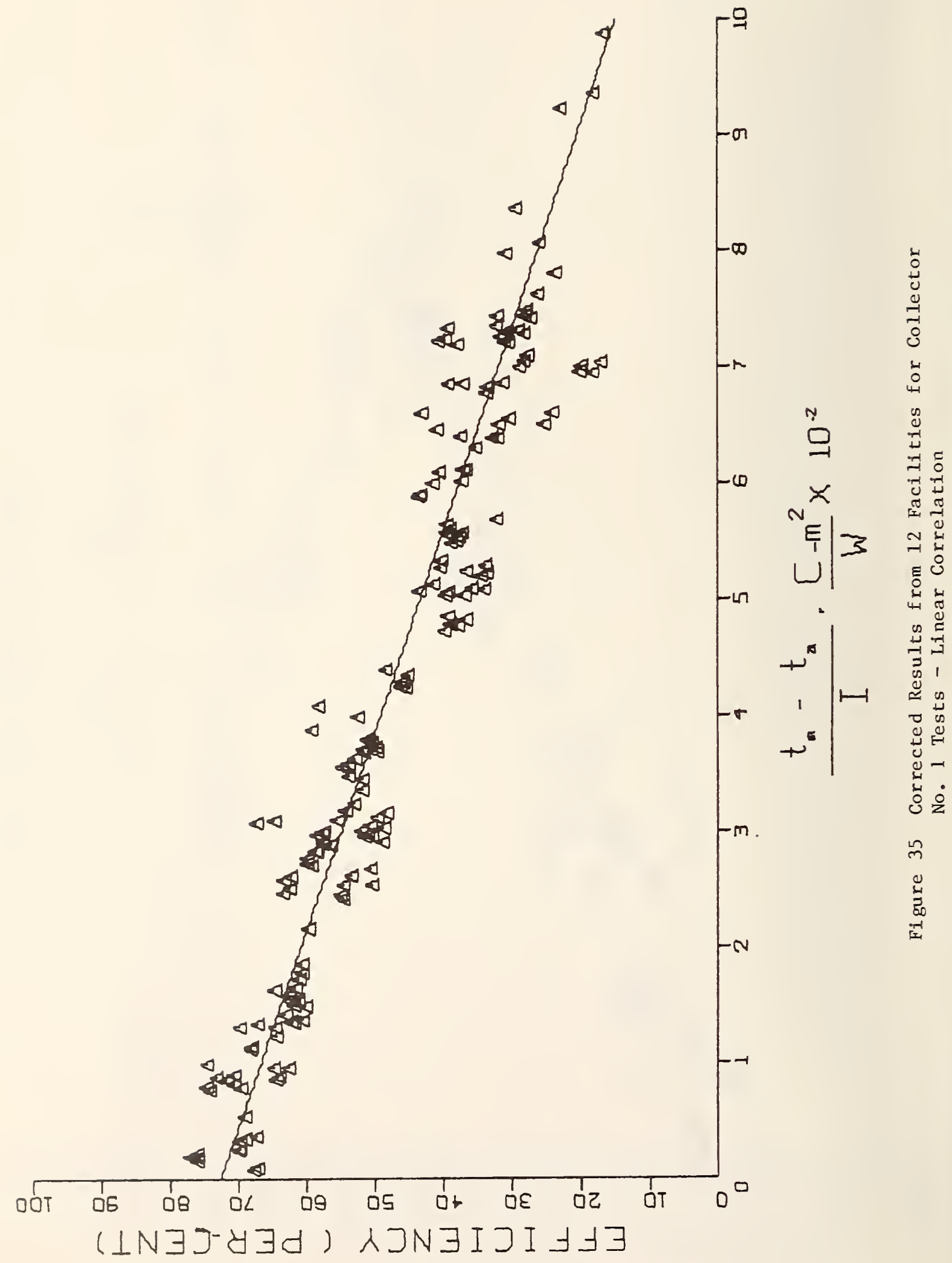




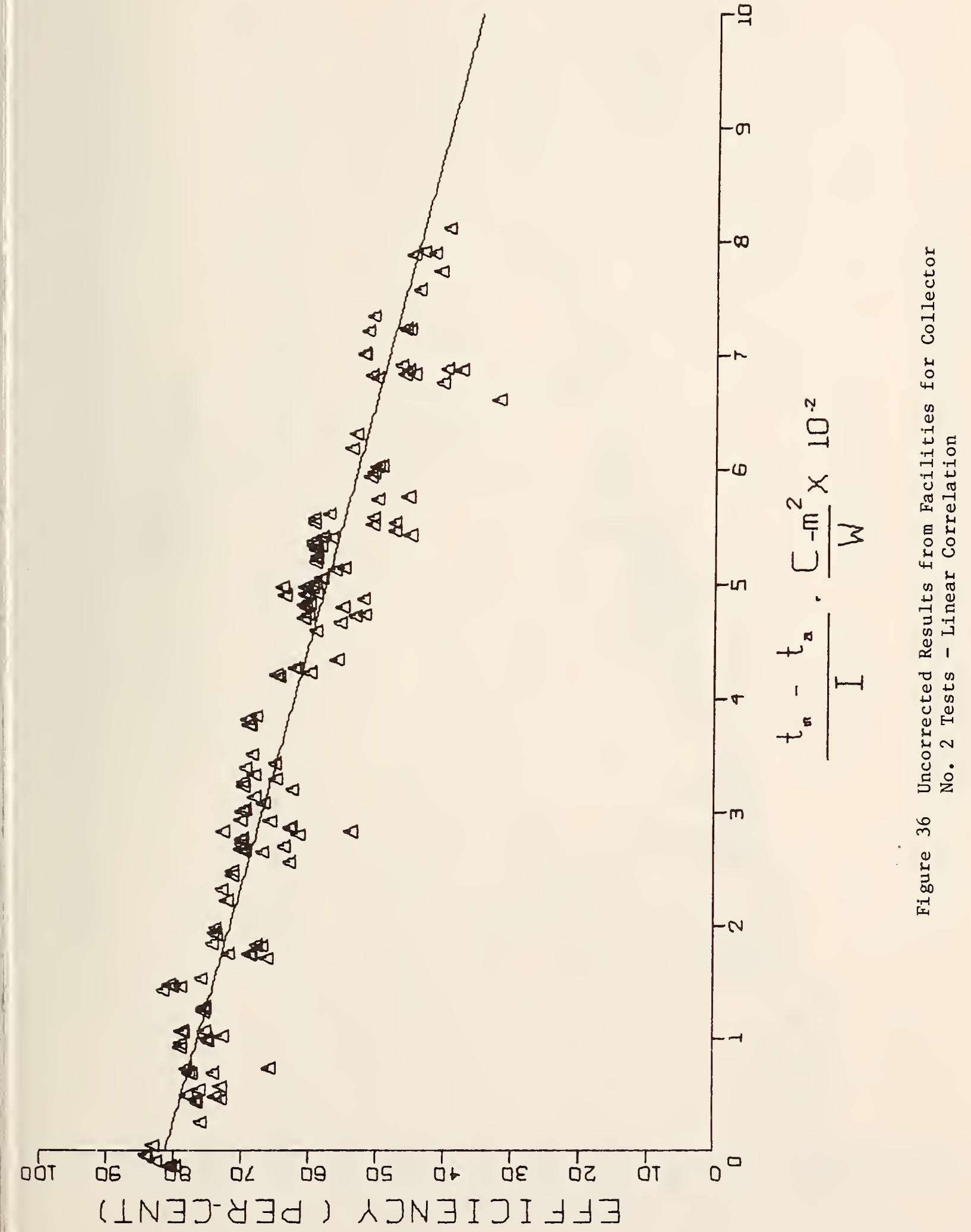




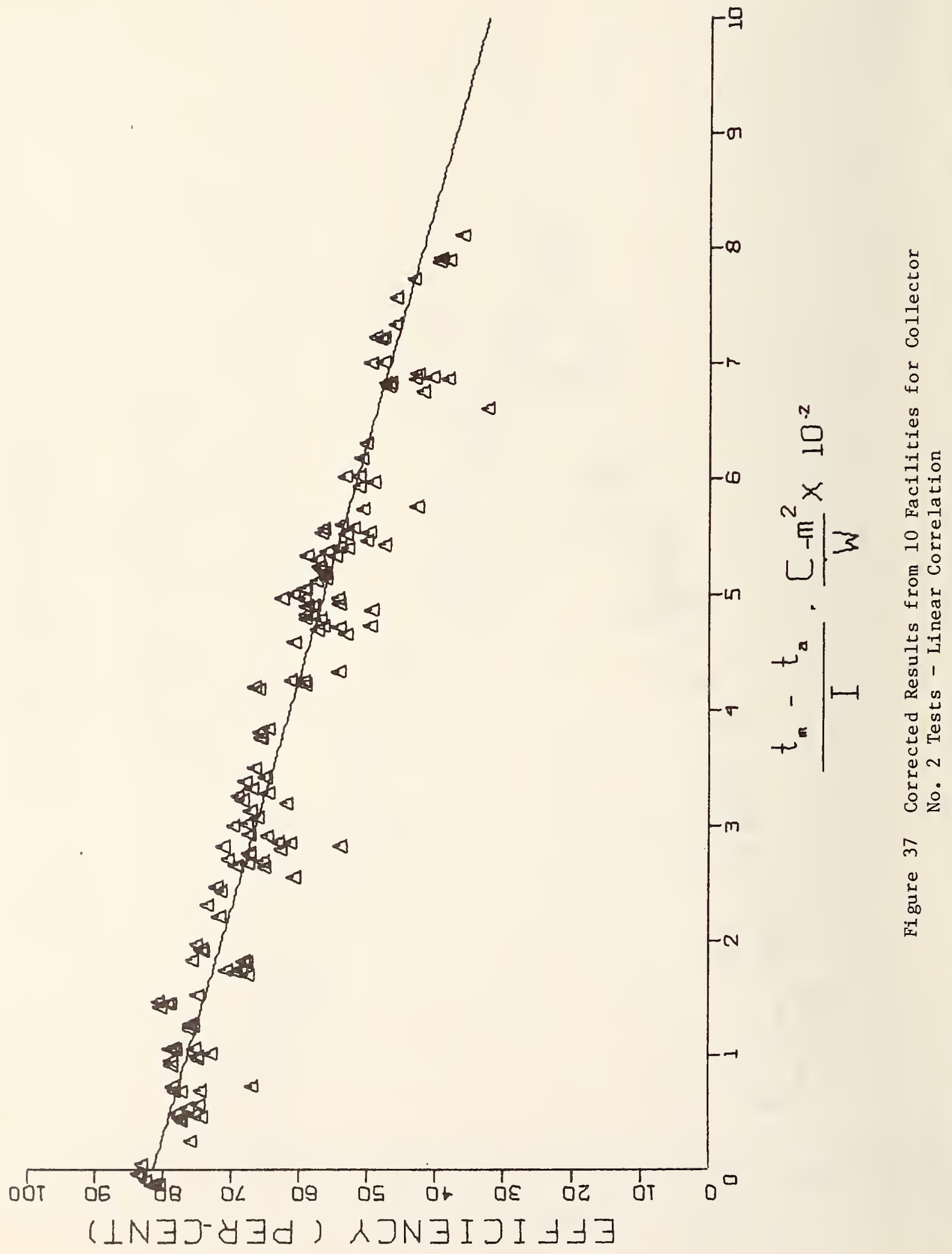




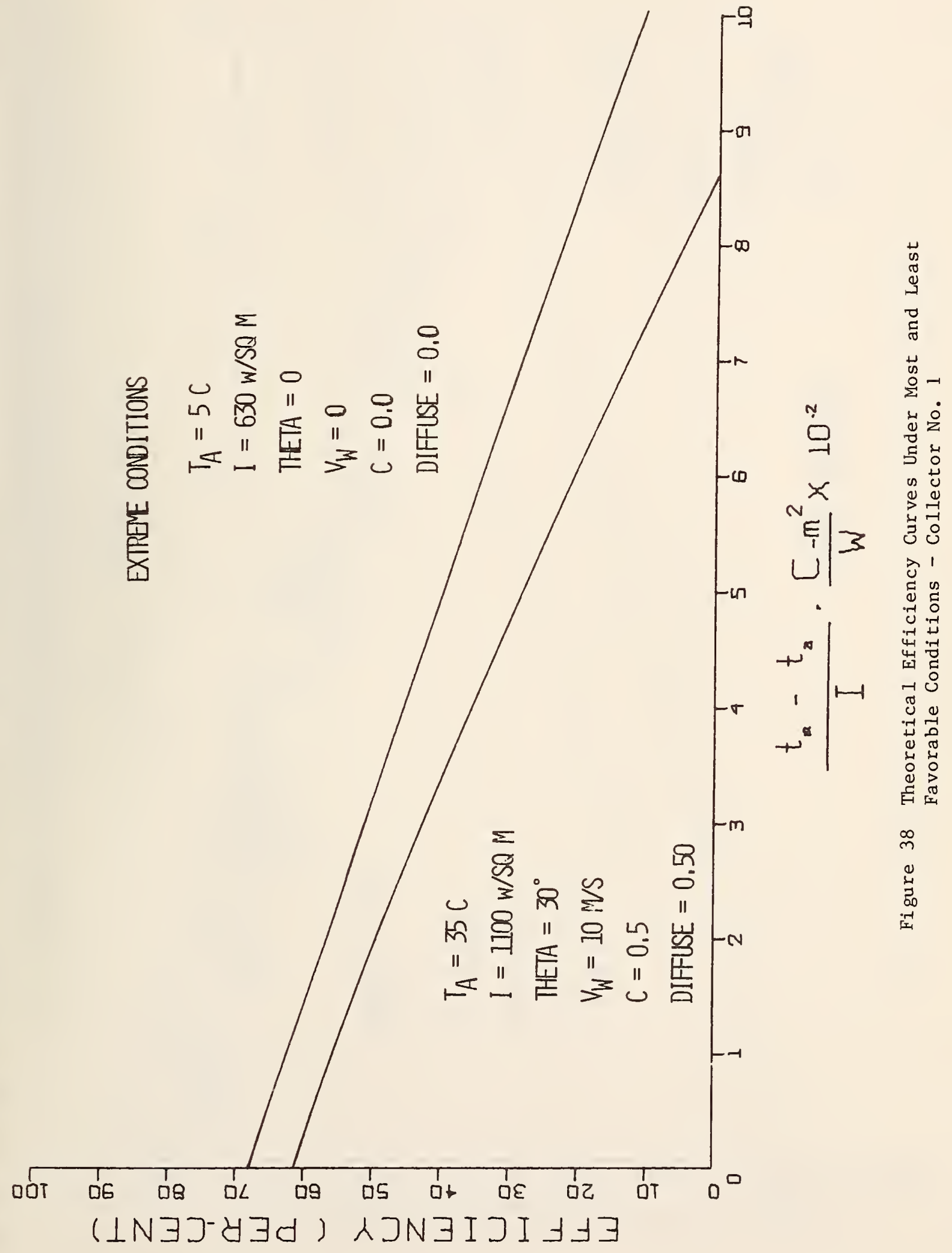




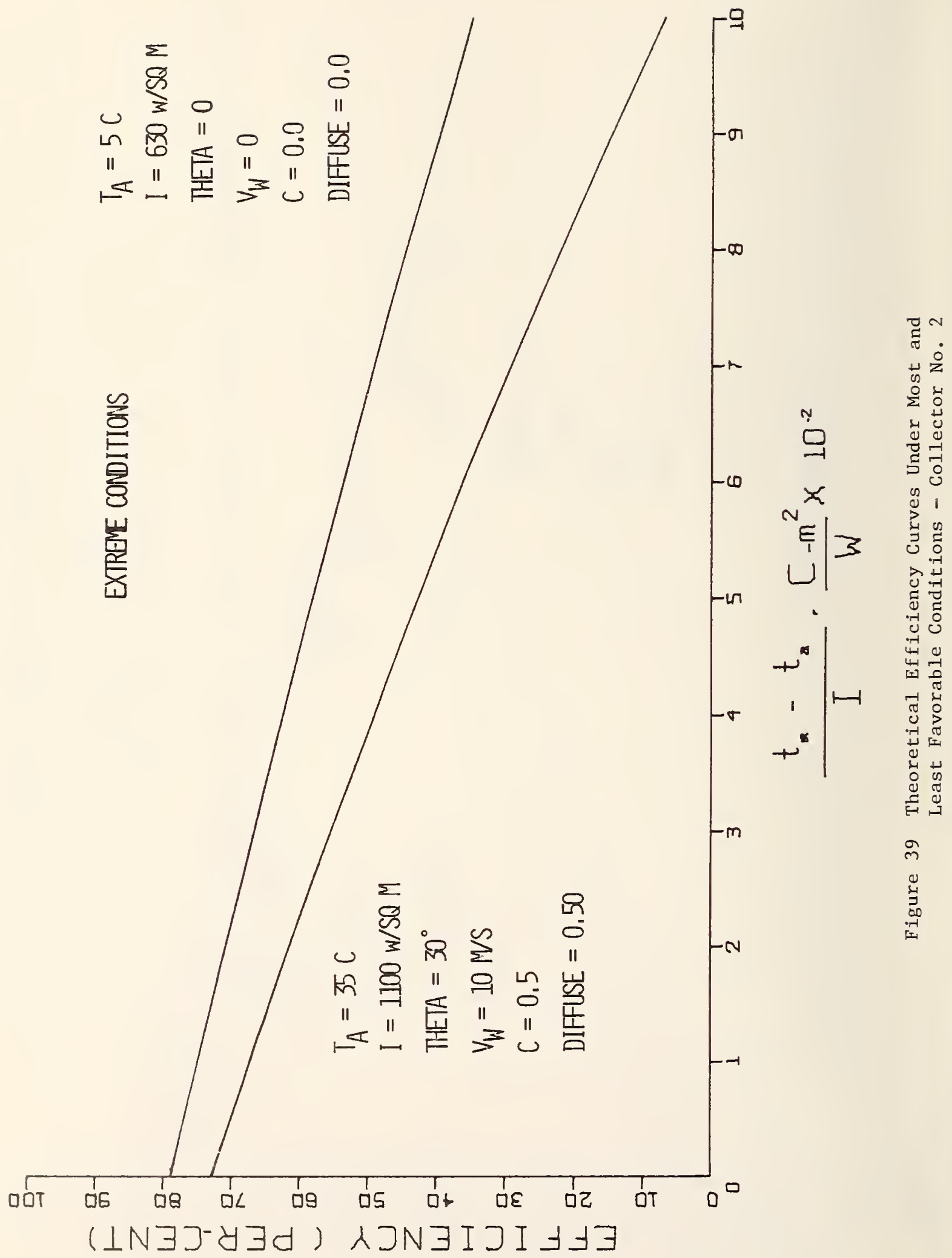




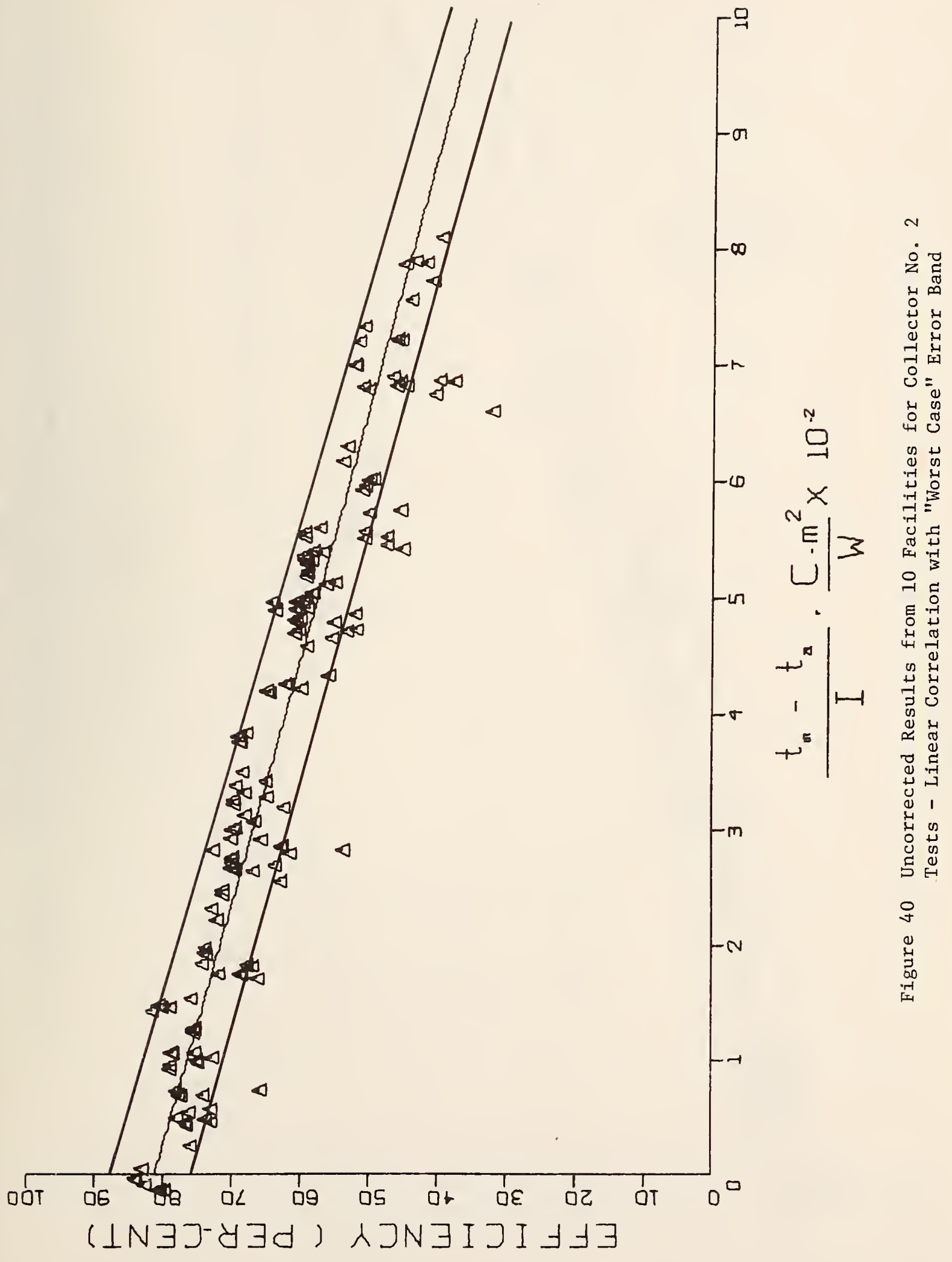




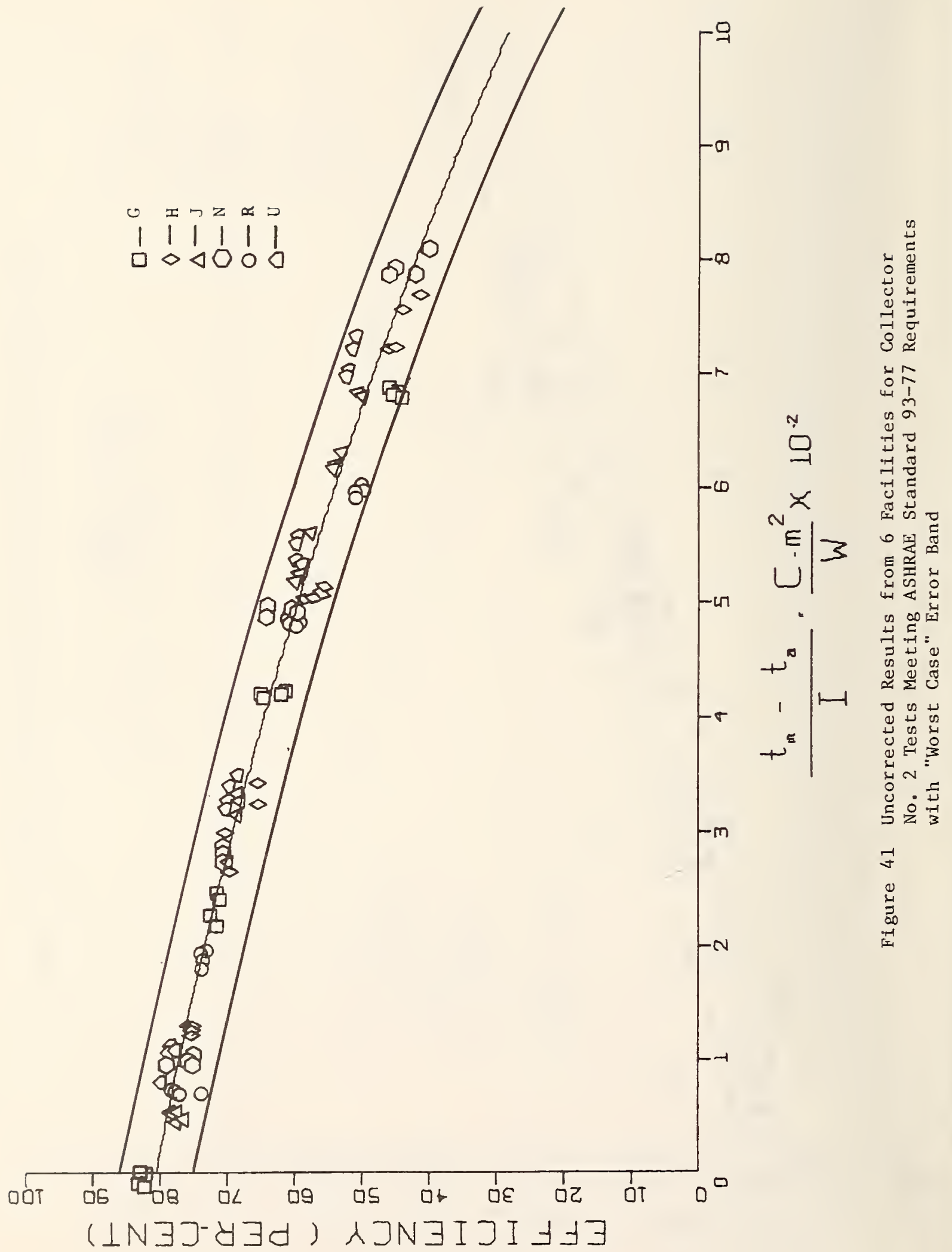




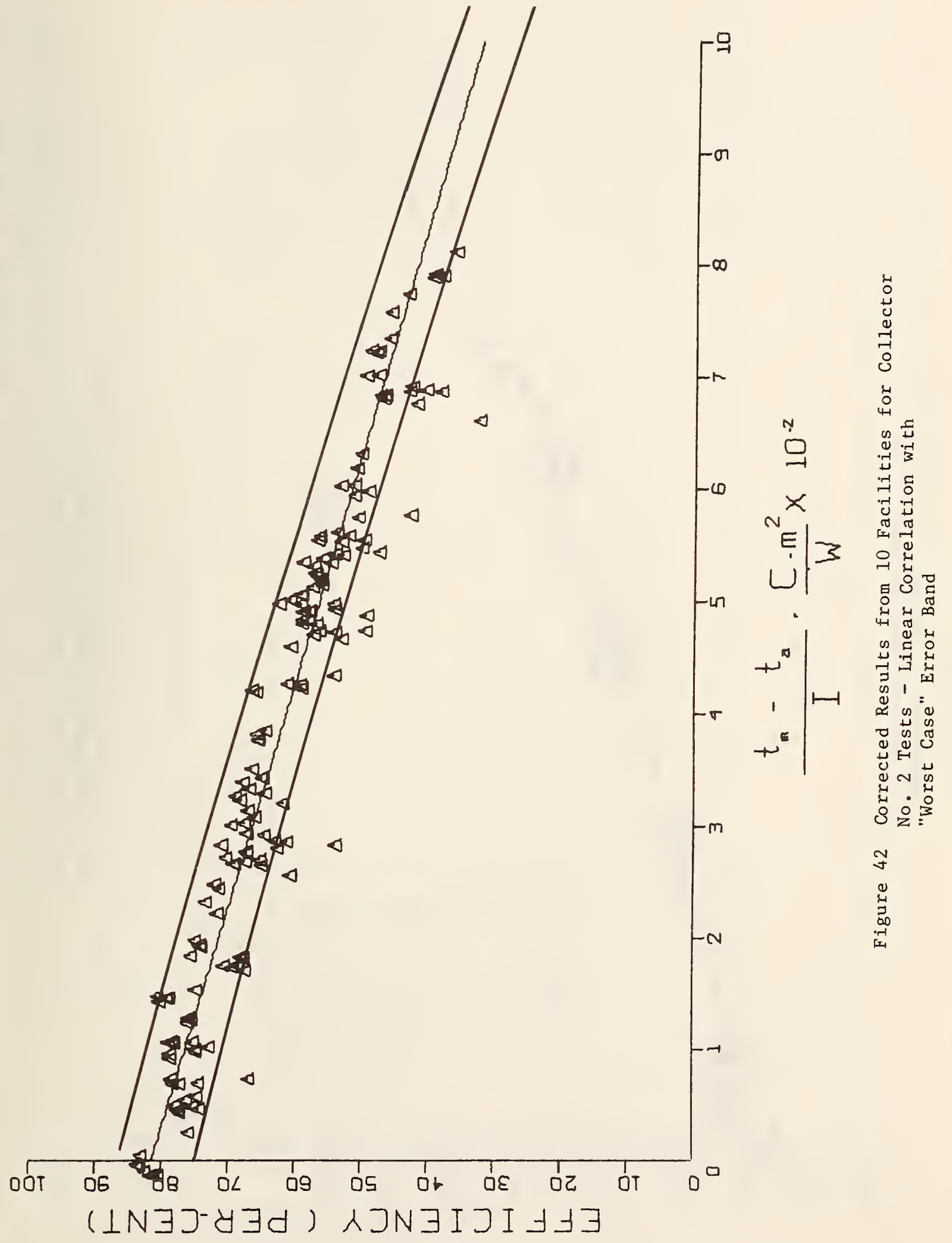




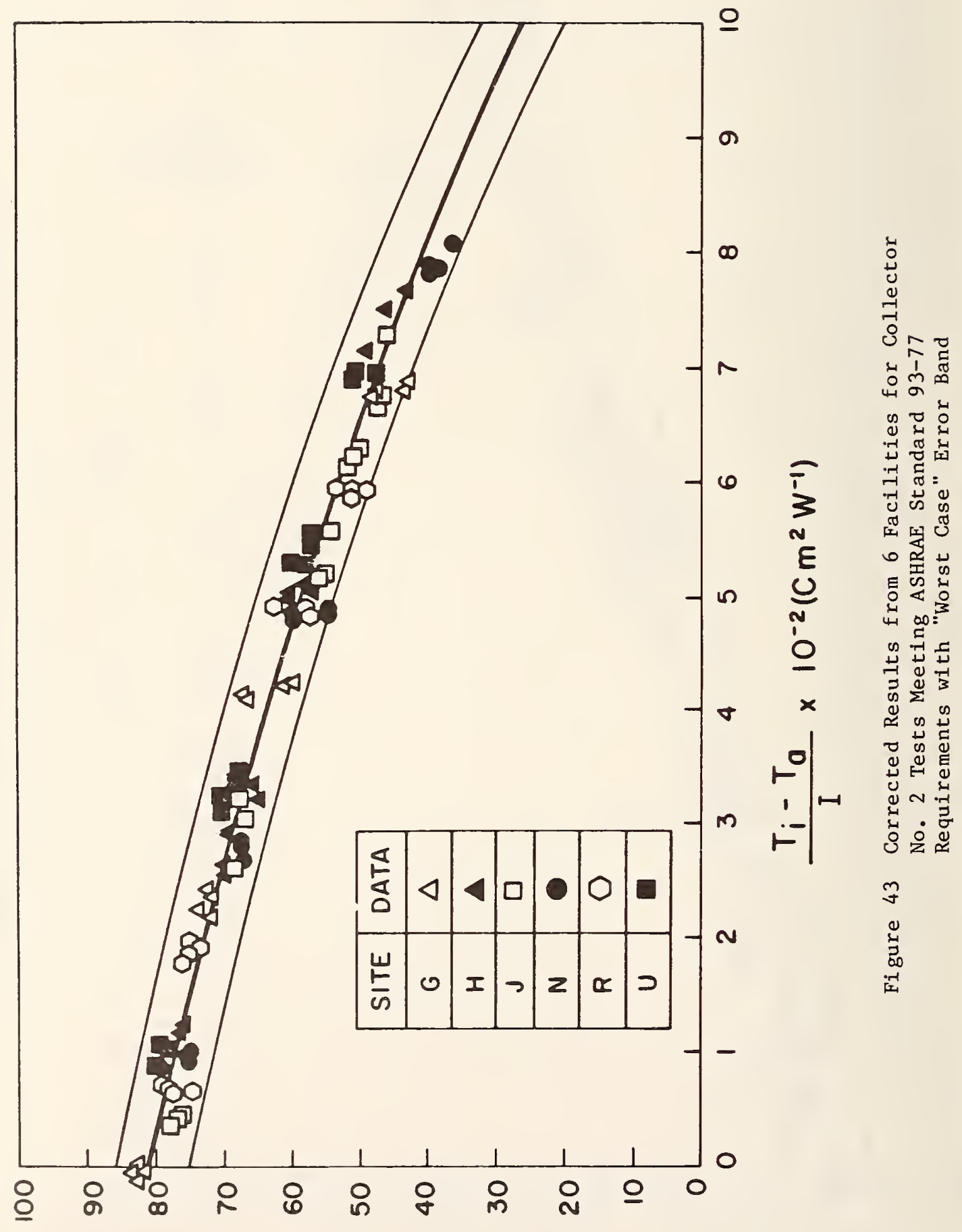




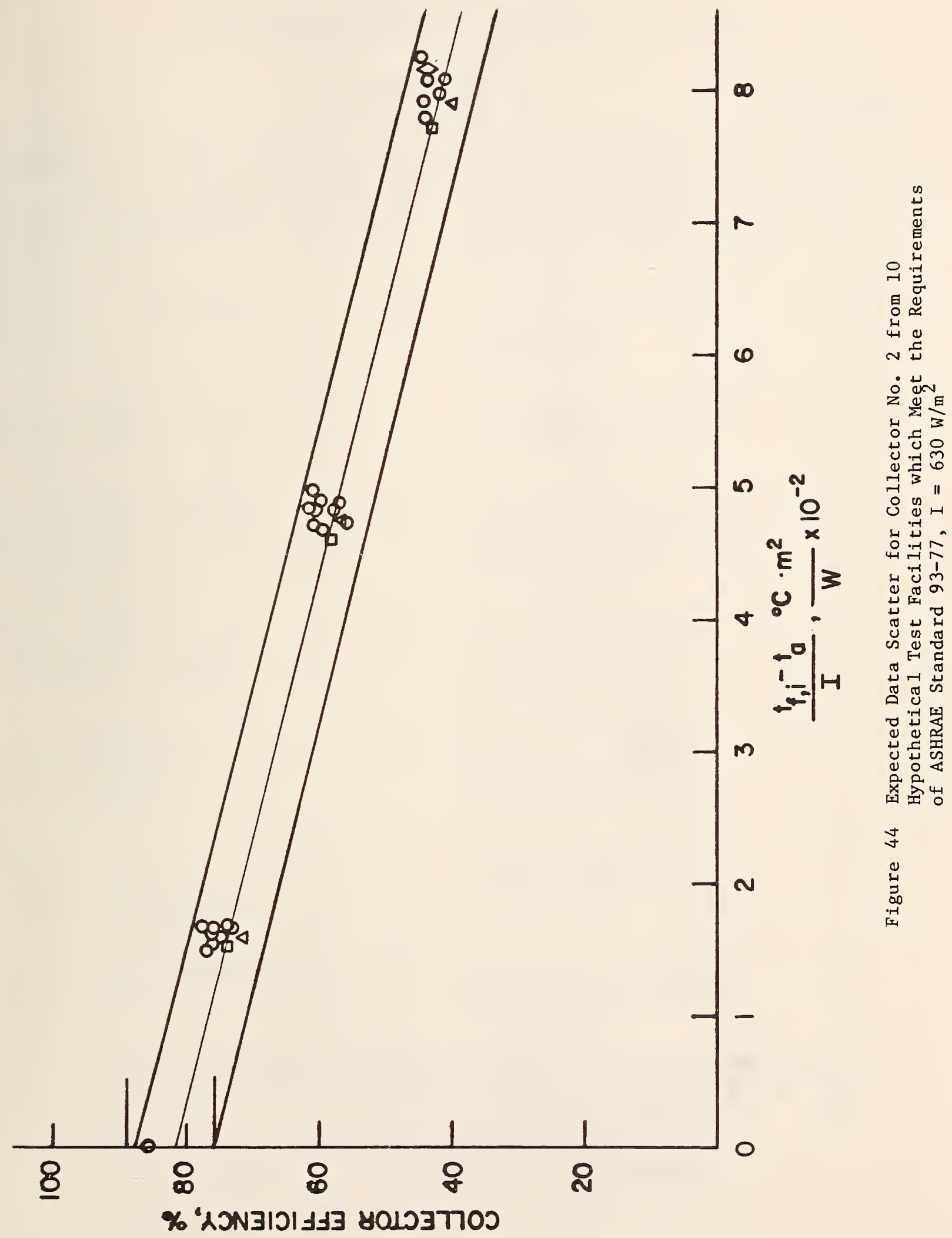




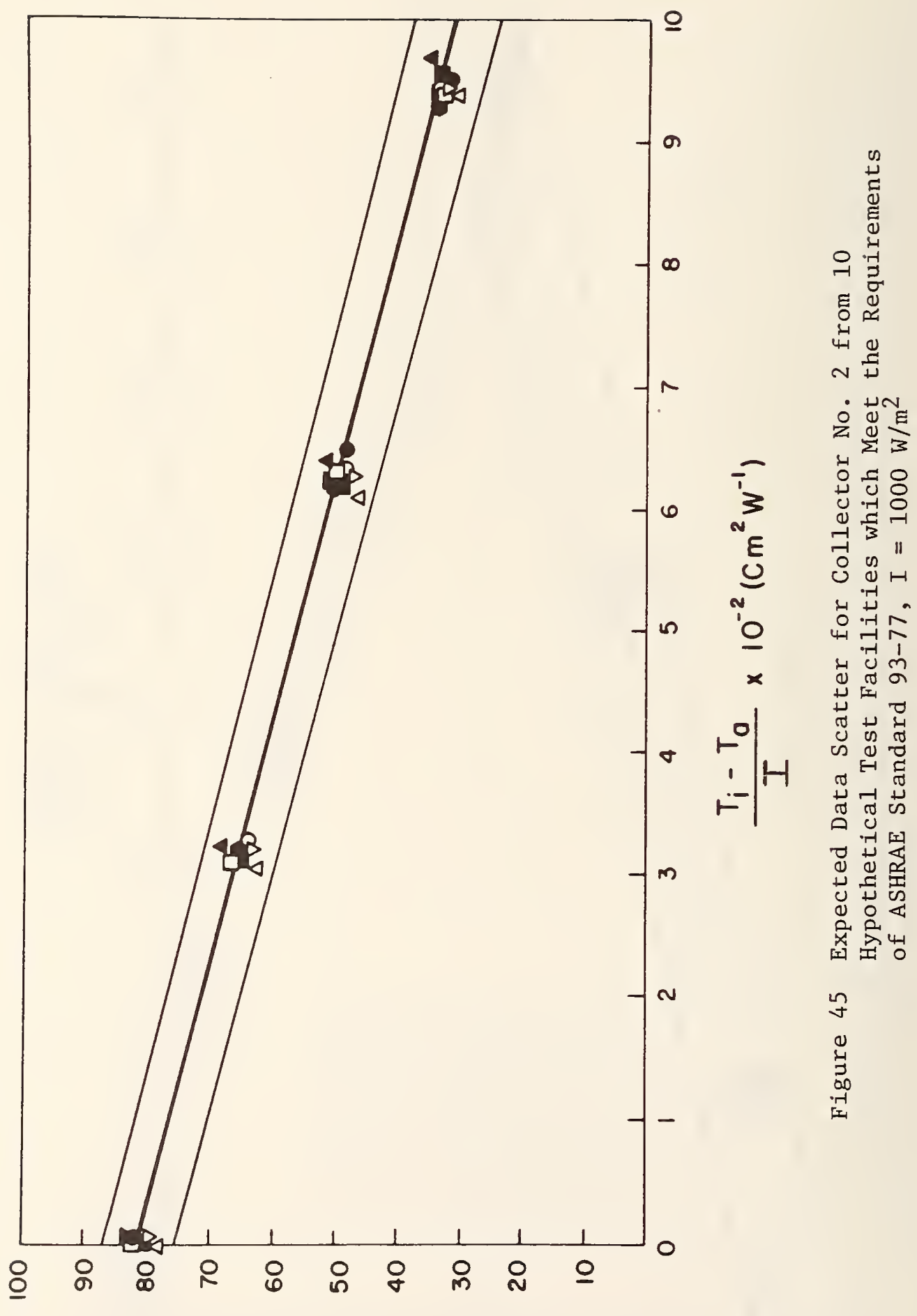

( 
Appendix A

List of Round Robin Participants

Collector

\#1 \#2

Collector

非非 2

Organization

Organization

Arthur D. Little, Inc.,

Cambridge, Massachusetts

W. David Lee

Arizona State University

Tempe, Arizona

B.D. Wood

Chamberlain Manufacturing Co. X Waterloo, Iowa

J.D. Morris

Desert Sunshine Exposure

Tests, Inc.

Phoenix, Arizona

G. Zerlaut

Honeywe 11, Inc.

Minneapolis, Minnesota

J.D. Kopecki

Lennox Industries, Inc.

Marshalltown, Iowa

David C. Lee

Lockheed Palo Alto

Research Lab

Palo Alto, California

K.L. Marsha 11

Martin Marietta Corporation X X Denver, Colorado

J. Kidd

New Mexico State University X X Las Cruces, New Mexico

H.L. Conne 11

X $\quad \mathrm{X}$

X X

X $\quad \mathrm{X}$

X X

$\mathrm{X} \quad \mathrm{X}$

X X

Conne11 $\begin{array}{ll}\text { PPG Industries } & \mathrm{X} \\ \text { Pittsburgh, Pennsylvania } & \end{array}$

M. Barker

University of Dayton X X

Dayton, Ohio

H.E. Smith

University of Florida $\mathrm{X} \quad \mathrm{X}$

Gainsville, Florida

E.A. Farber

University of Tennessee X X at Chattanooga

Chattanooga, Tennessee

L. Russe 11

University of Texas $\quad X \quad X$

at Arlington

Arlington, Texas

T.J. Lawley

University of Utah

X X

Salt Lake City, Utah

R.E. Boehm

University of Tulsa X X

Tulsa, Oklahoma

B.V. Ketchum

University of Miami

Coral Gables, Florida

$\mathrm{X}$

L.E. Poteat

University of Califor- $\quad X \quad X$

nia at Los Angeles

Los Angeles, California

H. Buchberg 
Appendix A (cont.)

\section{Collector}

非非

Organization

Virginia Polytechnic

X $\quad \mathrm{X}$

Institute and State University

Blacksburg, Virginia

W.C. Thomas

Civil Engineering

$\mathrm{X}$

Laboratory/NCBC

Port Hueneme, California

E. Durlac

National Aeronautics

$\mathrm{X}$

and Space Administra-

tion

Lewis Research Center

Cleveland, Ohio

F. Simon

University of California

X $\quad \mathrm{X}$

Los Alamos Scientific

Laboratory

Los Alamos, New Mexico

S.W. Moore

Nationa1 Bureau of Standards $\mathrm{X}$ X Washington, D.C.

J. Jenkins 
Data Used in Analyzing the Effect of Environmental Conditions

Data, measured results, and the theoretical analysis for each test facility are given in the following tabulations. "XPARM" is the abscissa of the collector efficiency curve as reported in terms of mean fluid temperature. The theoretically determined parameters are shown below the measured values. "PARM" entrees are the corresponding abscissa values of the collector efficiency curve in terms of the inlet fluid temperature. 
2

5

I $\alpha$

แU

(3)

$\bigcup \rightarrow$

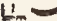

U.

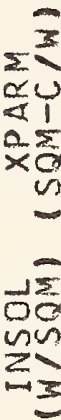

4

$4=$

$\lim _{\rightarrow \infty}$

IIVHLI OHMN

$\cdots \cdot \bullet \cdot \bullet \cdot \cdots \cdot \cdot \cdot \cdot$

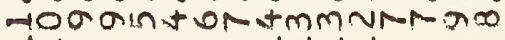

Noom-

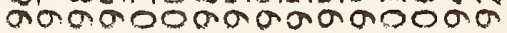
$-1+1$

$\rightarrow-1$

2

Q⿻

$=2$

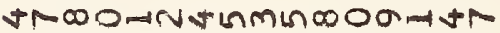
............

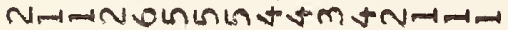

- 0.00000000000000

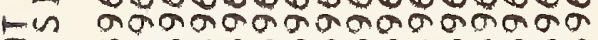

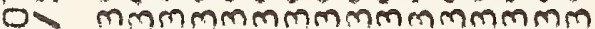

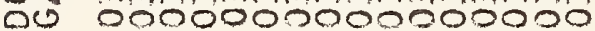

Ix .............. - 0000000000000000

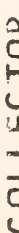

$\sum_{-\infty}$

DOHANmmmocogonath

OMRODO0.0000000MNMm

NNNWMMMMD 0.0 .000000

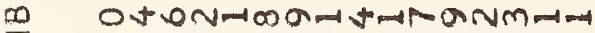

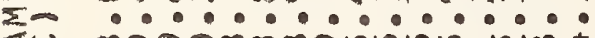

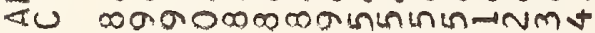

$\rightarrow$

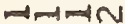

$\rightarrow-1 \rightarrow-1 \rightarrow-1$

w- 0000000000000000

o. Ous inininining 西

ñ

$\pi-3$

แU⿺

ID

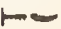

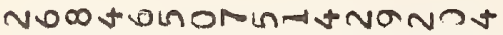

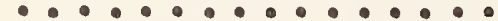

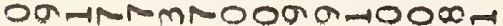
$\rightarrow$ HIN $\rightarrow-1=N$

$\underset{\alpha}{\alpha}$
$\Sigma$

$\alpha 0$

ax

Q.ut

-

$>$

$\bigcup_{1 \rightarrow \infty}^{\infty}$

40

40

U1)

$>$

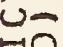

UL

un

แ4)

$>$

U⿻

mu

UI

ut

$>$

U一

$\rightarrow a$

$4 x$

$4 u$

네

$\alpha$

4

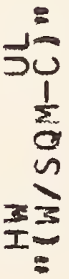

$>$

जে

10

$\frac{2}{\alpha}$ andontmathongona

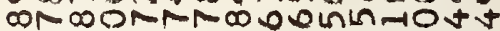

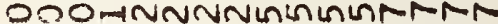
0000000000000000 $\cdot \bullet \cdot \cdot \cdot \cdot \cdot \cdot \cdot \cdot \cdot \cdot \cdot \cdot$ 0000000000000000

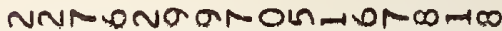
- : . . . . . . . mtttaracosagar cons

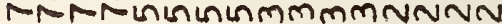

NCONDNGD OARNNMOOO คํำ:

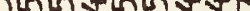

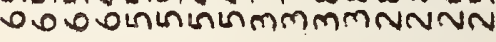

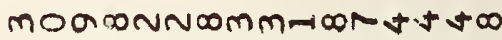
$\bullet \cdot \bullet \cdot \bullet \cdot \cdot \cdot \cdot \cdot \cdot \cdot \cdot$

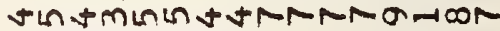
0 0 0 oL

mtornownta $0-10 m a \sigma$

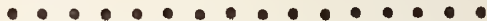
NM tma Oa MANIR⿴囗十 IIMmMmNmmN

-1न

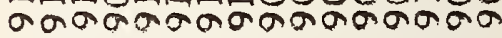

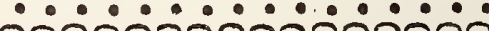
0000000000000000

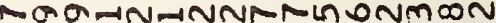

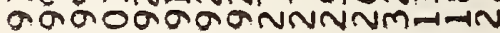

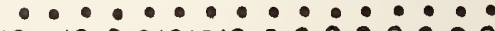

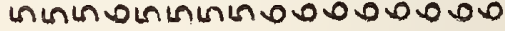

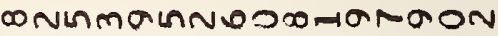
$\bullet \cdot \bullet \cdot \bullet \cdot \bullet \cdot \bullet \cdot \bullet \cdot \bullet$

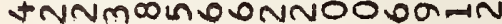

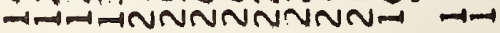

H-MNDON+ $\bullet \bullet \cdot \bullet \cdot \bullet \cdot \bullet \cdot \bullet \cdot$ $-m m+N \rightarrow-n m N \rightarrow \infty$ OL

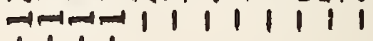
1111

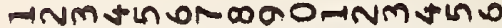

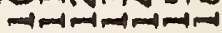




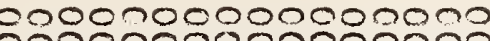
w

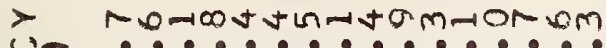
U. ............ La 山一 4II

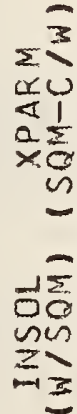

$4-$

$\sum_{i=1}^{20}$
$-\infty$
00
$5 x$

$\frac{0}{2}$

Z $m-$ $\rightarrow$

$+-$

$u$

$>$

$\sum$

$\sum \sqrt{13}$

$\infty$ $+$

w-

20

Qui ind

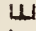

$\frac{\alpha}{0}$

$\ll-$

10

บUแ

IO

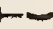

$\frac{z}{\alpha}$

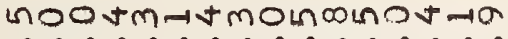
NNNNMMMM0.0000000000

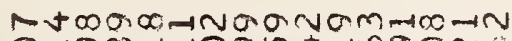
O-0.00न100.

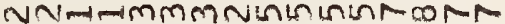
ofoonolonomón 0000700000000000

$\bullet \cdot \cdots \cdot \cdot \cdots \cdot \cdot \cdot \cdot \cdot \cdot$ OMa-mines Lnvinแกisivint

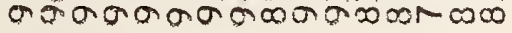

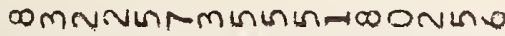
- *...... manino OOAMRNNGOO-

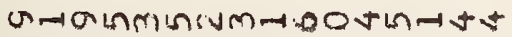
-

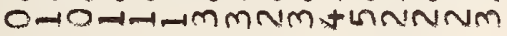

NNNMEMNNM+.000 ONDO

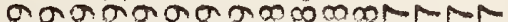
$-1-1-1-1-1-1-1-1-1-1-1+1-1$ 0000000000000000 $\cdot \bullet \cdot \bullet \cdot \bullet \cdot \cdot \cdot \cdot \cdot \cdot \cdot \cdot$ 0000000000000000

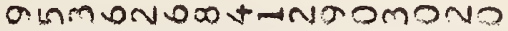
-............ $m+i n h m m m+-1 \times m \pm a D-10$ $\rightarrow-1-1 \rightarrow-1 m-N$ NNN $\rightarrow$ VNN

0000000000000000

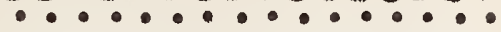

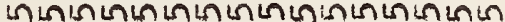
t t t t t t t t t t t t t t t

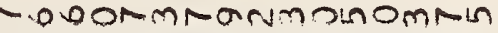
$\bullet \bullet \cdot \bullet \cdot \bullet \cdot \bullet \cdot \cdot \cdot \cdot \cdot$ NIT-NA-NENMIIMNNm

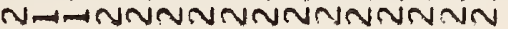

-Nmt

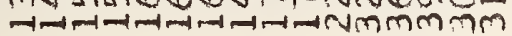

50
$\alpha=2$
01

$-$

$>$

$\bigcup_{-10}$

40

$4+$

3

10

4.5

un

$>$

$0-11$

UI

난

3

$\rightarrow a$

$4 \times$

$4 \amalg$

$\propto$

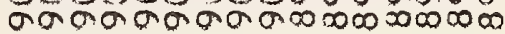

$\bullet \cdot \cdot \cdot \cdot \cdot \cdot \cdot \cdot \cdot \cdot \cdot \cdot \cdot$ 0000000000000

- romoDolnoontanava

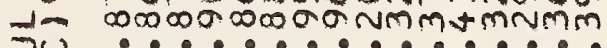

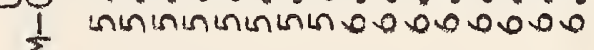
$\sum$

in

$I=$

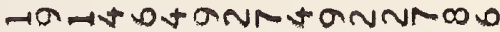

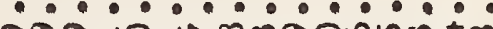

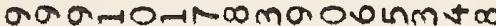
$\rightarrow-1 m-1-1-1 \times N=1+1-1$

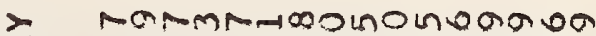
$x \rightarrow \cdot \cdot \cdot \cdot \cdot \cdot \cdot \cdot \cdot \cdot \cdot$

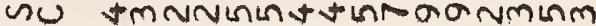
$-111111111$

$\frac{2}{\alpha}$ 


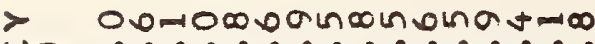

U $\bullet \bullet \bullet \bullet \cdot \bullet \cdot \bullet \cdot \bullet \cdot \bullet \cdot \bullet$

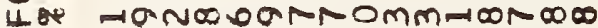
1 - IntLAt L

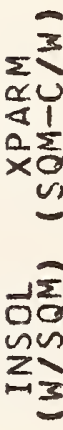

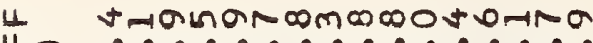

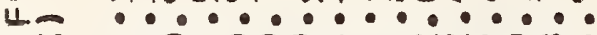
W $=0$ -

$0-\pi-1-1-1=1-1-1=1-1-1-1$

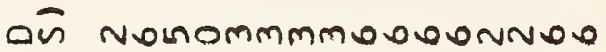

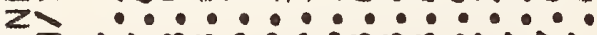

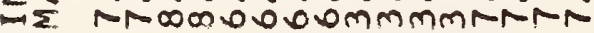

Q

Q⿻日土

皮
$Z$ $+\infty$

-

$1-$

$\infty$

$\sum ?$

दु

$\rightarrow$ 1

ய-

20

Qu

$\pm 0$

42

$-\infty$

wu

I

E。

$\sum$ NoN No th-t tobmtinim

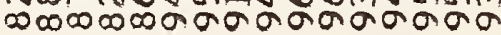

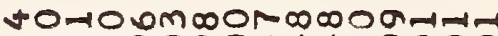

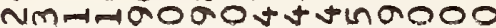
mmmmNm Nmmmmm Nmmm 000000000000000 • • • • • • • • • • • • 0000003000000000

moO $\circ \cdot 0 \cdot 0 \cdot 0 \cdot 0$ $0 \infty 0$ - 00 NmLnLñotst

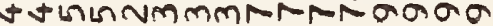

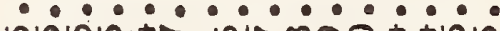

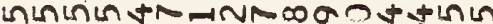

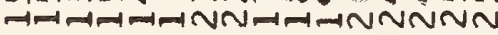

0000000000000000 $\cdot \bullet \cdot \bullet \cdot \cdot \cdot \cdot \cdot \cdot \cdot \cdot$. 0000000000000000 जмmलмm

00000 D000000000 . . . . . . . thmon

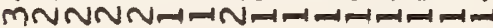

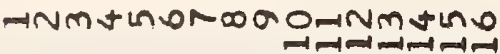

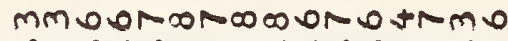

$\frac{\sum}{1} \frac{1}{x}$

a.

$>$

$\lim _{10}$

10

40

แU⿺

$>$

U- inmotranaraningaro

mo $\bullet \cdot \cdot \cdot \cdot \cdot \cdot \cdot \cdot \cdot \cdot \cdot \cdot \cdot$

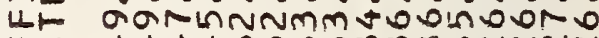

Un tattoo to

$>$

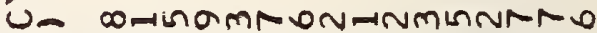

जU L.I coaptrimminowinmmN

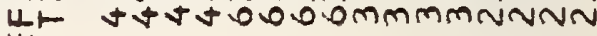

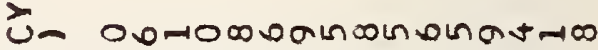

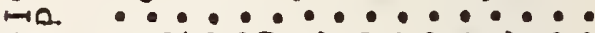

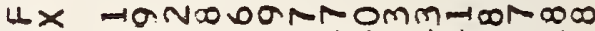
UL IntLI t

$\frac{\infty}{4}$

NNNNNNNNNNNNA-A-1aráagagararáa ..............

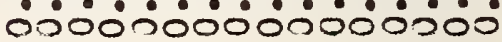

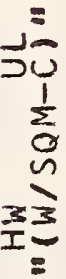

OMNAMNONJIRDNDODO

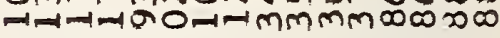
$\bullet \bullet \bullet \bullet \cdot \bullet \cdot \bullet \cdot \bullet \cdot \bullet$

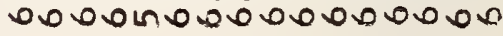

$-100-10000+7+\pi-100$ $\bullet \cdot \bullet \cdot \cdot \cdot \cdot \cdot \cdot \cdot \cdot \cdot \cdot$ mtcorararagammtt mmmm NN VN

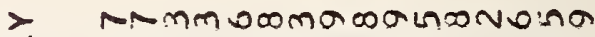

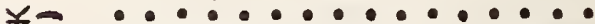
nu NNNNMOONOAM -11111 त्मात्

$\frac{Z}{\alpha}$

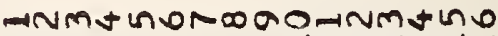
$\rightarrow \rightarrow-1 \rightarrow-1=$ 


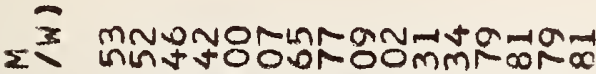

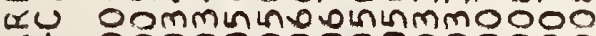
\& 1 00000000000000 nx $\cdot . \cdot \cdot \cdot \cdot \cdot \cdot \cdot \cdot \cdot \cdot \cdot \cdot \cdot$ $\times 0000000000000000$

n

0

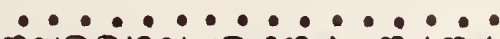

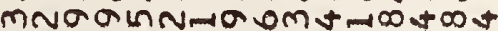

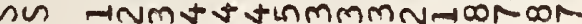

z- o00000000000\%ana

$-1$

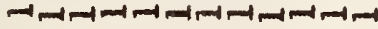

4 OLNNA-AONITOMLII

U.

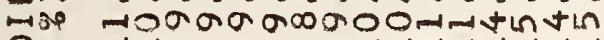
$0-$-1

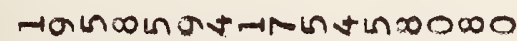

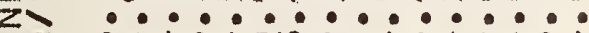

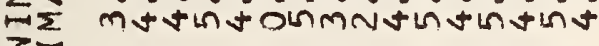

omotrammprinthata

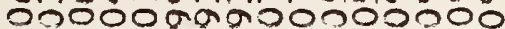

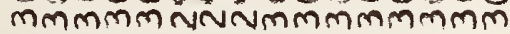
D0000000000000 $\cdot \cdot \cdot \cdot \cdot \cdot \cdot \cdot \cdot \cdot \cdot \cdot \cdot \cdot$

แ⿻

0

$\rightarrow 0$

$<-$

$-0$

닌

IO

10

$\sum_{\alpha}^{Z}$

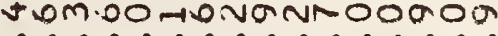

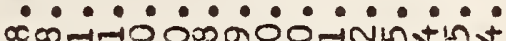
NN:D O0 $00000000 \mathrm{mmmm}$

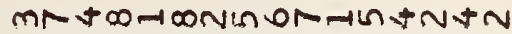
. . . . . . . . . . -NDOOODO00 NNNNM NMmMmmMmmMm

0000000000000000 . $\cdot . \cdot \cdot \cdot \cdot \cdot \cdot \cdot \cdot \cdot \cdot$

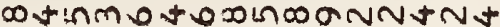

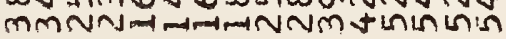

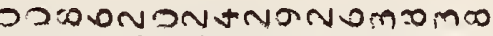

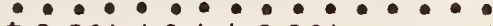
$+m M N-1 m+ \pm m M N-1-1-1-1-1$

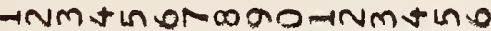

\section{$>$}

$\Sigma$

$x a$

$\approx x$

au

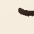

$\bigcup_{\infty}^{\infty}$

40

แU

U-

$>$

U)

40

Uin

แ⿺辶

3

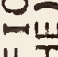

U.

$u_{4=-}$

$\underbrace{}_{10}$

$\bigcup_{\operatorname{li} \infty}$

$4 x$

4.U

யथ

$\alpha$

NNNNAN-1-ANNNNNNNN

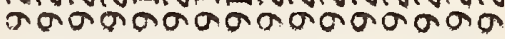

4

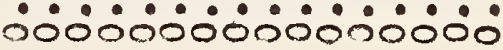

20

$\frac{1}{x}$

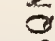

$3=$

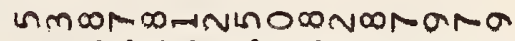
- *.......... mant notomononmono

$=$

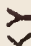

我

$\rightarrow \infty$

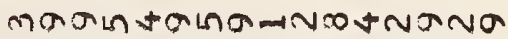

$\frac{z}{\alpha}$

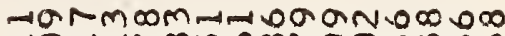

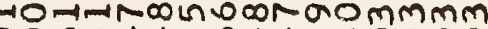

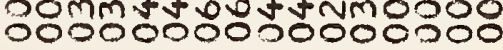

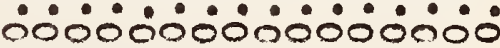

MoOMWONMAMOOO Mt-4t -..........

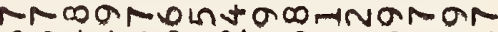

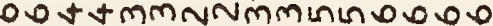

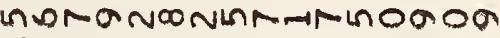

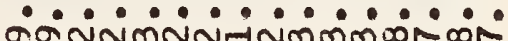

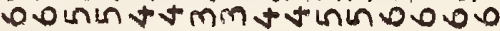

minoocotmoalnolnot ot

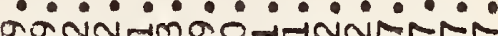
00เกนกษ

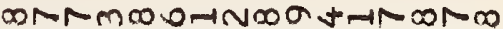

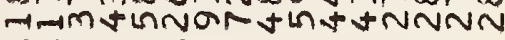
- *.......... 0000.000000000000

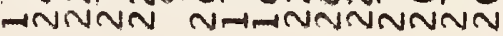
-...........

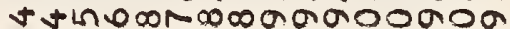

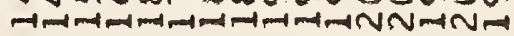

-nMtinonogonnmino 


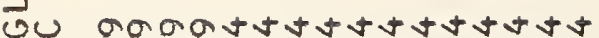

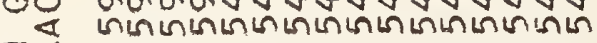

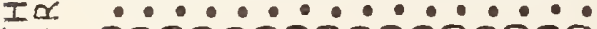
Fu D0000D0000000000 ш

$>$ MNDWOALNOMOOHNOD U

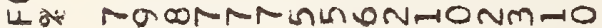
it inininintt U. 3
23
$\frac{1}{2}$
0
$\times 3$
0

arragmintagonocom z- $=$ -

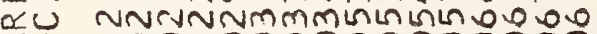
\& 1 000000000000000 $0 \leq . \cdot \cdot \cdot \cdot \cdot \cdot \cdot \cdot \cdot \cdot \cdot \cdot \cdot$ 0000000000000000

15

in

$\geq \sum_{i=1}$

$\cdots \cdot \cdot \cdot \cdot \cdot \cdot \cdot \cdot \cdot \cdot \cdot \cdot$

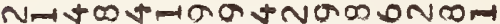

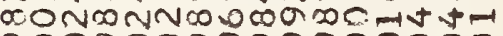
000000900000000

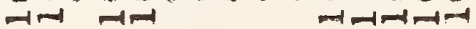

L MONOOOOMNOUNOMNLI $4=\ldots \ldots \ldots . \cdots \cdots$

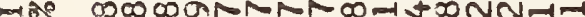
$0-\quad-1=1-1-1-1 \times N N=1-1$

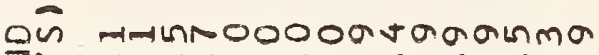
Z mI mmtNatatthat00-0

- NMWONNTNOMNNt+LIR

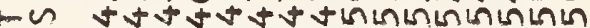
Q NNNNNNNNNNNNNNNN DU 0000000000000000 Y $-$ 2000000000000000

$\frac{0}{a}$

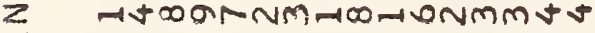
$\rightarrow=0.0 . \cdot 0$.

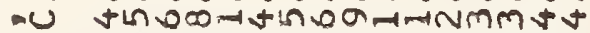

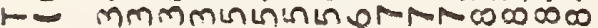

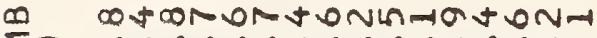
EI ..............

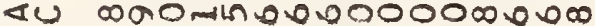

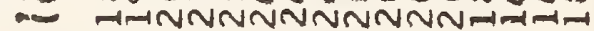
$+$

แ-

Q0

Qu

$-10$

D00000000000000

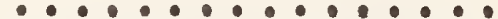

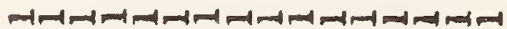

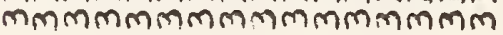

$<\frac{10}{10}$

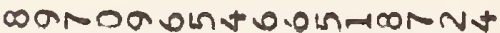

แU⿺

-

ID -

$1-$

$\frac{z}{a}$

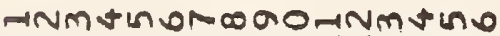

$\sum a$
$\alpha=x$
$\alpha u$

Qu

$>$

$\bigcup_{\infty} \infty$

40

U.

W

$>$

$3=$

U.

Lis

แU

$>$

$\bigcup_{11}$

U. $I$

$\frac{1}{4}$

$>$

$\bigcup_{\substack{0 \\ 0}}$

40

U山

U1

$\alpha$

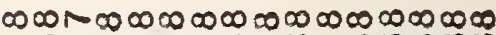
$\infty \infty \infty \infty \infty \infty \infty \infty \infty \infty \infty \infty \infty \infty \infty)$

4 0000000000000000

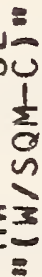

ㄴ.

NamoLOMNO-NQTHN

:!!!!!.!.!.

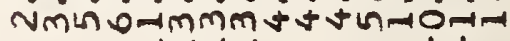
$\rightarrow \rightarrow-1$

11

$\sum_{\alpha}^{Z}$

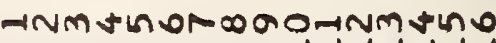
$\rightarrow-1-1=1-10$ 
$>$

00

I ơ

14

Ui)

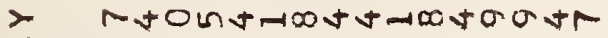

$u=\cdots \cdot \cdots \cdot \cdot \cdot \cdot \cdot \cdot \cdot \cdot \cdot$

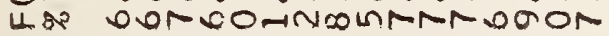

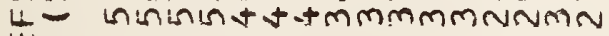

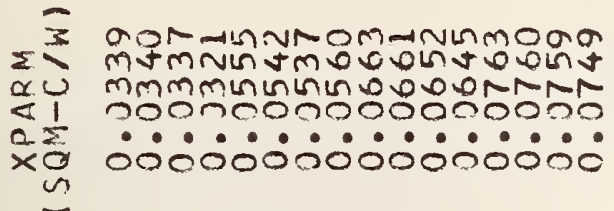

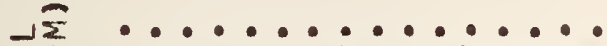

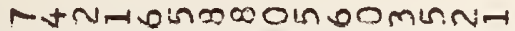

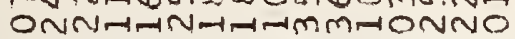

$\geq 30000000000000000$

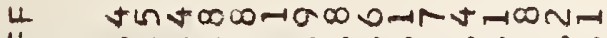
H. 1 .............

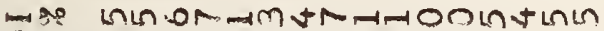

0- न1-1म-1म-1मन-1

$\sum_{z=}^{0}$

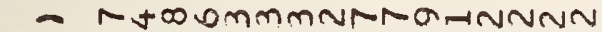

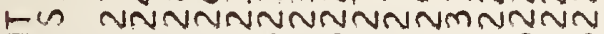

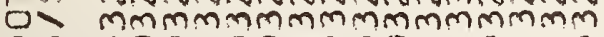
0 000000000000000

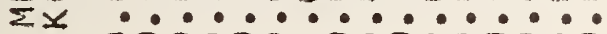
- 000000000000000

$Z$

10

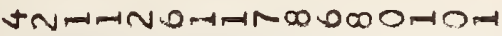
$\bullet \cdot \bullet \cdot \bullet \cdot \bullet \cdot \bullet \cdot$ DNNNNUNImmmmLInLIR $-\infty$

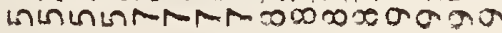

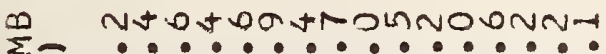

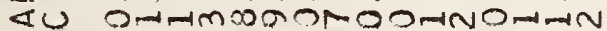
- NNNNAFNANNNRINTN $\vdash$

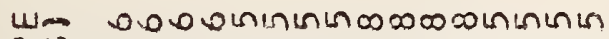
0 ดய 든 *

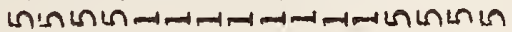

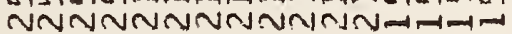

\&- D000000000000000

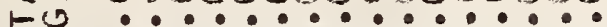

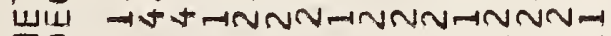
ID $\rightarrow$ मा ना मा F⿻

$\sum_{\alpha}$

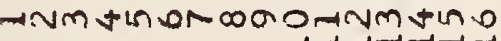

$\frac{5}{00}$
$\frac{1}{x}$

$>$

U⿺辶一兀

10

แU

Li.

$>$

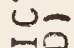

4.

$4 n$

2

3

$4 I$

4 -

$>$

U.

$4 \times$

는

Ш山-

$\alpha$

4

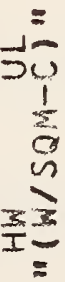

$>$

$x-1$

जu

$1-$

$\frac{z}{x}$
$\mathrm{O}-\infty+\infty+\infty+\infty \mathrm{NONN}-\mathrm{NO}$

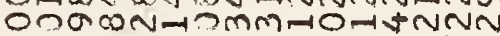

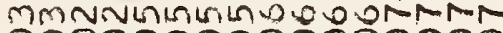

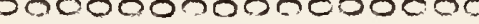

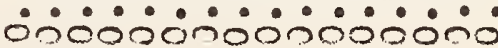

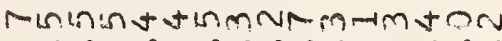

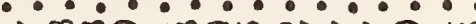

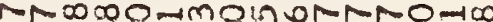
unlnint totmmmmammo

D.OH-H -

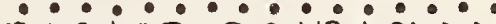
$m m m+00-19 m+14+\infty N-N$ inthint

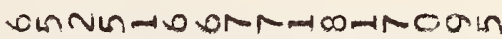
-............ NNNNOOAmInininint 00

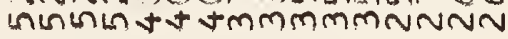

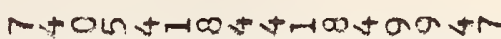
$\bullet \cdot \cdots \cdot \cdot \cdot \cdot \cdot \cdot \cdot$ ODNDO-NDOINANDOOOM

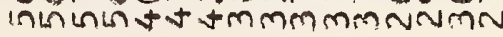

Dn0000000000000

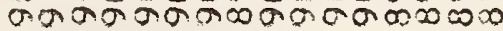

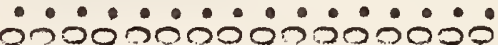

ONCON+DORN NiRGAGR

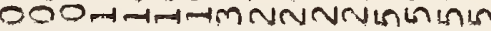

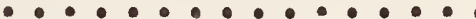
- 000000000000000

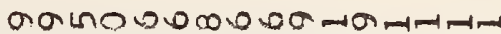
. . . . . . . . . .

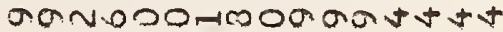
-1 $\rightarrow 1=1$ -

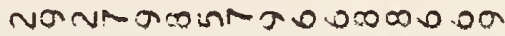
*........

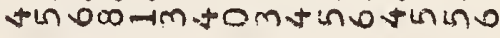

-NMm 
$>$ ONTOONOTEM⿻00000 U- .............. L of NMmNaOCLIRTNGONAN

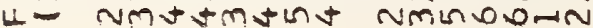

Li

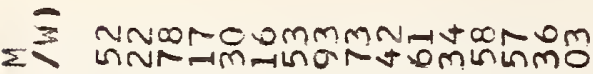

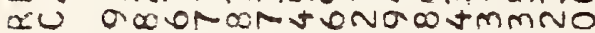

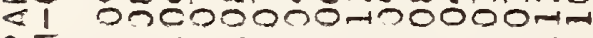
c.5 $\dot{2} \cdot \dot{0} \cdot \dot{0} \cdot \dot{0} \cdot . \dot{0} \cdot$ s

$\frac{15}{00}$

in

$\sum_{i=1}$

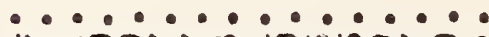
AN

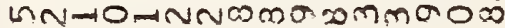

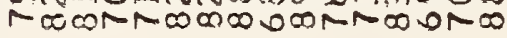

4

$\prod_{1 \rightarrow 0}$

0000000000000000 $\rightarrow-1-1=-1-1-1-1-1 \rightarrow N=1$ gus
$\geq \Sigma$
$3=$

OTMNNDHRDOONR-NN - * * * * * * * * GINNNANNAH TNNMNN

$\ln$

-1- $-1-1-1-1-1-1-1-1-1-1$

NNNNNNNNNNNNNNNN

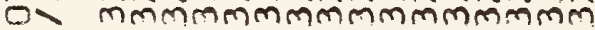

D0 000000000000000

$\sum \underline{Z}$ -

............

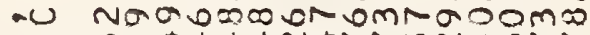
- OHAt

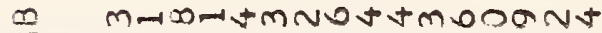

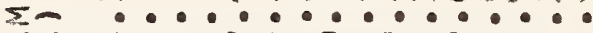

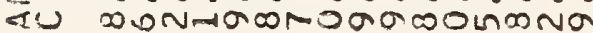
$-$

$\vdash$ $\begin{array}{lllllllllll}1 & 1 & 1 & 1 & 1 & 1 & 1 & -1 & 1 & 1\end{array}$

I 1

L:1-

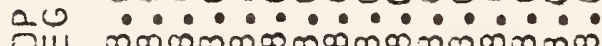

0000000000000000 jo $a t a t a t a t a t a t a t a t$

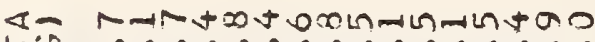

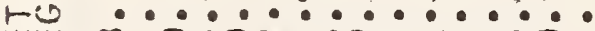
UW IO NNNMM-NAMAMN-MMm 10

$\sum_{\alpha}$ -Nm

$\Sigma=$

20

$<x$

$2 L$

$>$

$\omega \sim$

$\square \propto$

40

110

$>$

$\omega_{10}$

$\rightarrow 0$

4 is

Ш一-

$>$

U一

-

$4 I$

$4 F$

$>$

U.

$\rightarrow 0$

4 W

U1)

$\frac{x}{u}$

0000000009000000

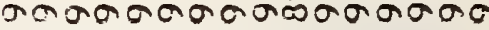

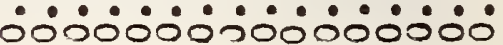

5
50
$\frac{1}{0}$
un
$3=$

$>$

$x-$

जu

r-

$\sum$

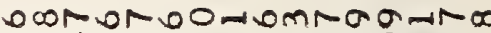

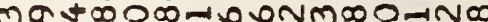
ar o 000 Ot ONa $00000000-100000-10$ í0000000000000'

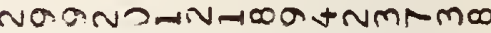

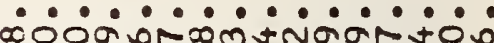

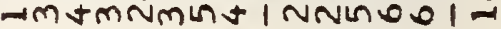

tMง

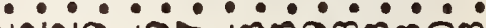
NNNO-AT-NMNNNAm-1-1 thin-

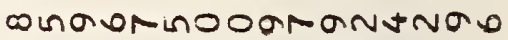
$\bullet \cdot \bullet \cdot \bullet \cdot \cdot \cdot \cdot \cdot \cdot \cdot !$

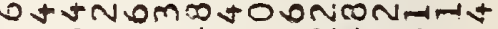

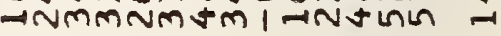

ONTOORANAMDO DaAm NMMN-1OEIRTONO ONMN NmstmsLt NmISbo-n

O-dOH tNMONmGIIIAMN

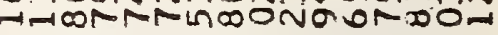
$\cdot \bullet \cdot \bullet \cdot \bullet \cdot \cdot \cdot \cdot \cdot \cdot \bullet \cdot$

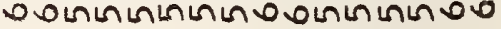

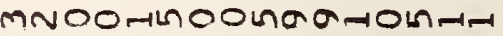
-. $\cdot \cdot \cdot \cdot \cdot \cdot \cdot \cdot \cdot \cdot \cdot$ +D00 TNO ONOOA ONAt NNa-1-1-1-1-1-1NN-1-1-1-1-1

NMOOLNNAMNRNA-1THR $\cdot \cdot \cdot \cdot \cdot \cdot \cdot \cdot \cdot \cdot \cdot \cdot \cdot$ HNNL DHM⿻上OOLMN-100 MmNNmMmmmmMN

1111111111111111

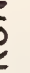


$>$

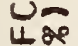

4

U1)

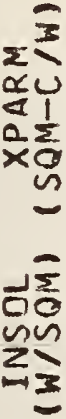

4

$u_{-10}$

o-

oñ

$\sum_{i=1}$

$x=$

-n

잉

De

21

$\frac{0}{2}$

2

$\sum_{13}$

r-

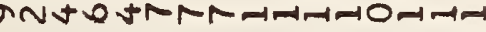

............

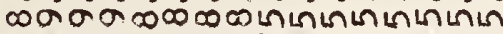
NNNNIIIILIRMAMN 0.000

$\infty$

$\sum \sqrt{6}$

$\rightarrow$

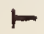

$\omega-$

00

ow

जo

amanaGantw00m TM -........... mo. mmmtt

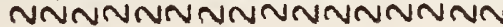

0000000000000000

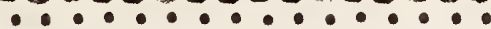

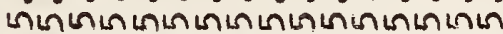
Aatatatatatatatata

$<-\frac{10}{15}$

$-0$

世ய

IO

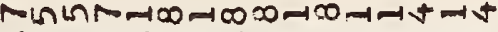
$\bullet \cdot \bullet \cdot \bullet \cdot . \cdot 0$

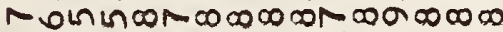

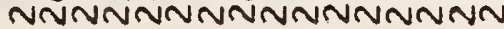

$\frac{Z}{a}$

HNm thon $\sum \frac{1}{\alpha}$
$\alpha \mu$
$\alpha \mu$

a

$>$

$\omega$

40

40

$>$

$3=$

$\rightarrow 0$

岂佂

U一

$\rightarrow=$

UЕ

$>$

$\bigcup_{-10}$

$4 x$

U.

แU

$\alpha$

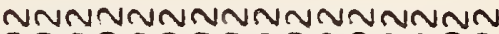

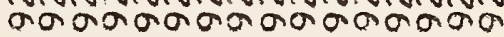

u $\cdot \cdot \cdot \cdot \cdot \cdot \cdot \cdot \cdot \cdot \cdot \cdot \cdot \cdot \cdot$ 0000000000000000

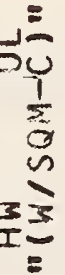

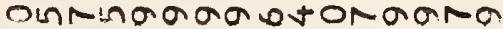

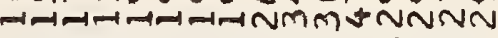
10000000000 0000000000000000

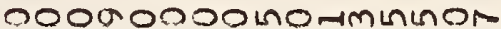
............. O0.000000NOT+NNO0

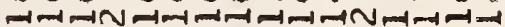

- InDNNininanininotanin

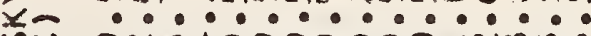
जu anmtarasoonimmm

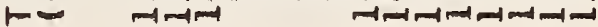

$\frac{2}{2}$

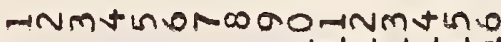




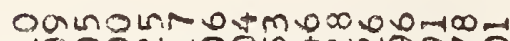

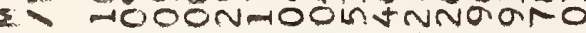
\&

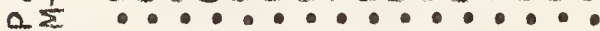
$\times$

$\bullet \bullet \bullet \bullet \bullet \bullet \bullet \cdot \bullet \cdot \bullet$ NNR DoO 0000000000000200

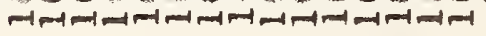

u.

0000000000000000

U. $\bullet \bullet \cdot \bullet \cdot 0^{\circ} \cdot \bullet \cdot \bullet$

$-29$ $\mathrm{Nm}+\mathrm{m}+\mathrm{mm} 0.0 \mathrm{~N} \mathrm{NH}-1 \infty$

$\sum_{i=1}^{\infty}$

$m 5$

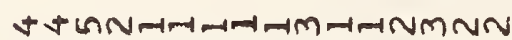

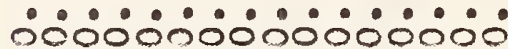

$-\bar{n}$

$0>$

00

$\Sigma \geq$

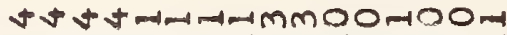

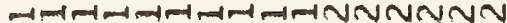

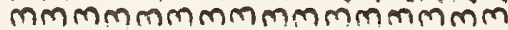
०00000000000200 .$\cdot \cdot \cdot \cdot \cdot \cdot \cdot \cdot \cdot \cdot \cdot$ 0000000000000000

Z NMMMON ㄱ...............

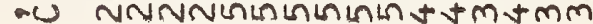
$1-$

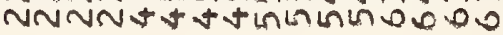

GNDMD OOMOINONMTOL

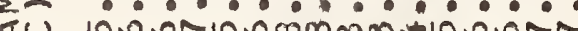

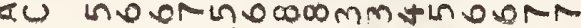
-

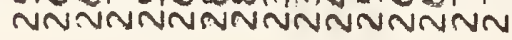

แ⿻

20

OU

$\rightarrow 0$

0000000000000000

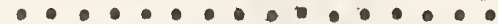
0000000000000000

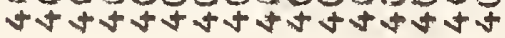

a 3

แบำ

ID

$F$

200000000000000

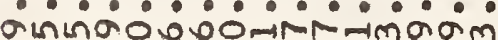

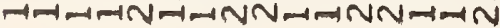

$\sum_{\alpha}^{2}$

-NMt

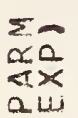

a.

$>$

$\omega-$

$=\infty$

140

W-

$>$

U一

4

Un

Wis

$>$

$\bigcup_{411}$

$\mu I$

UL

W-

$>$

U2

$\rightarrow 0$

$4 \times$

는

$\alpha$

$\frac{\alpha}{4}$

$=$
20
$\frac{1}{2}$
0
5
$\frac{1}{2}$

जo

MAOMNMMONARMOINGO m.!?!.?!?!.

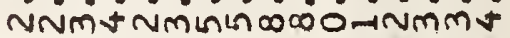

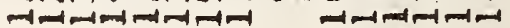

$\frac{Z}{\alpha}$

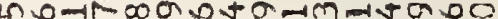

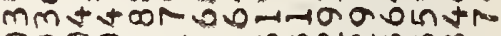
0000 -1-tmmrvrummm 0000000000000000

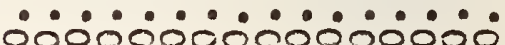
1111

NNMMMNMMMLO000000 $\bullet \cdot \cdots \cdot \bullet \cdot \bullet \cdot \cdot \cdot \cdot \bullet$ MNLno00-1t+LINANM-1-

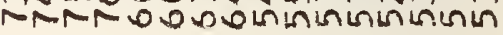

aOmbat-inhoO-HNPo

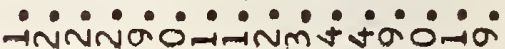
MMM H 0.00 แn

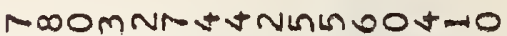
$\bullet \cdot . \cdot \bullet \cdot \cdot \cdot \cdot \cdot \cdot \cdot \cdot \cdot$

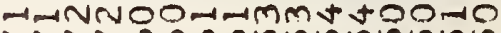

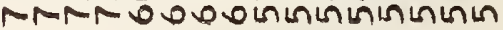

0000000000000000 min

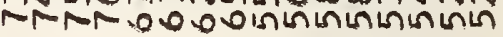

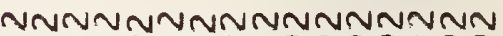

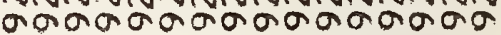

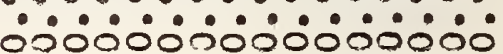

OONMR

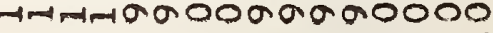

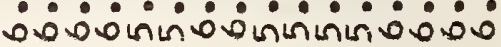

NNOL

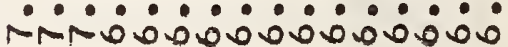

- $N m+10-\infty 00-10 m+n 0$ 
$>$

Wu 0000000050020000

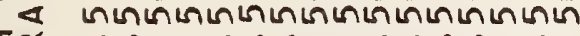

Ir

U

$>$

U.

$u$

U.

$\sum_{2}$

$=$

$\approx 0$

$0 \frac{1}{2}$

$\times 0$

$\sqrt{2}$

जo

zo

$-13$

-

4

4

$\rightarrow \infty$

$0-$

Q⿻日土

$\sum_{i=1}^{\infty}$

32

$-2$

0

$\sum x$

$-$

(1)

2

$-10$

$-\infty$

$\cdots \cdots \cdot \cdots \cdot \cdots \cdot \cdots \cdot$

$\sim m-m+ \pm N-10-1 m 0+-10$

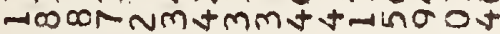
D00000000000000

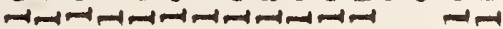

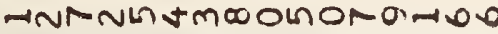

.$\cdot \bullet \cdot \bullet \cdot \cdot \cdot \cdot \cdot \cdot \cdot$ INNOONNNA+MUIM-1-t

$\rightarrow-1-1-1-1-1-1-1-1 N m m$

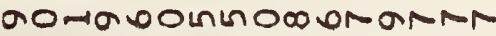
*... NMmNMNMmNNNOOOO

inm $-1-1-1-1-1-1-1-1 N 0 N-1-1-$

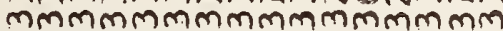
0000000000000000

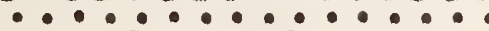
00000000000000

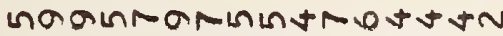
$\cdot \bullet \cdot \bullet \cdot \bullet \cdot \bullet \cdot \cdot \cdot$

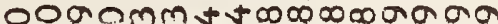

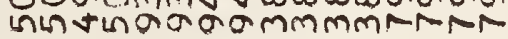

C NDEDTONNOOLNONDO

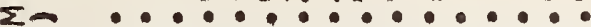

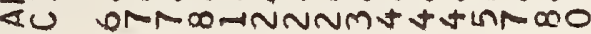

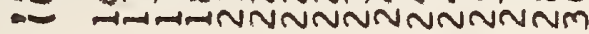
$\vdash$

U一

Q

口u

ñ

0000000000000000

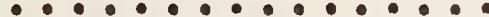

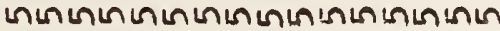

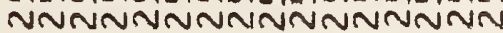

4- Ot+ON-INMMMmONNO

-10

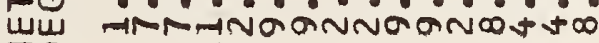
ID $\rightarrow$-1 $\rightarrow-1$-1

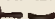

$\frac{2}{\alpha}$
$\Sigma=$

$\frac{2}{1} \frac{2}{x}$

0 ن

-

$>$

$\bigcup_{i=1}$

40

40

แ2

$>$

U.

$\rightarrow \infty$

His

แ-

$>$

$\bigcup_{\rightarrow 1}$

U1

41

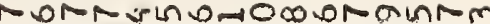
mogasogonmmmin?

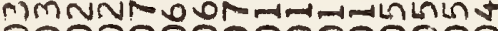
0000000000000000

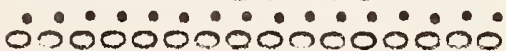

कMIIRONAOTWOMNOMD $\bullet \cdot \bullet \cdot \bullet \cdot \bullet \cdot \bullet \cdot \cdot$

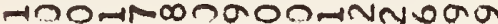

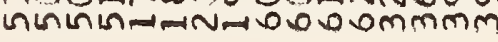

InMDONNNDOANDLNMN $\bullet \bullet \cdot \bullet \cdot \bullet \cdot \bullet \cdot$ -mMmgog

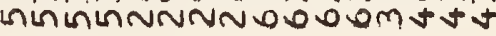

$\bullet \bullet \bullet \cdot \bullet \cdot \bullet \cdot \bullet \cdot$

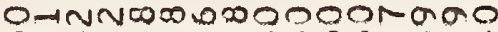

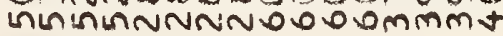

$>$

Un

TMNMNADRNOOHNONONN

10

$u x$

U.

OATOONMAODOONLAN

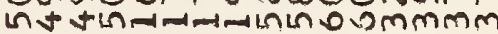

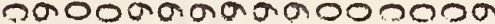
$\frac{c x}{4}$

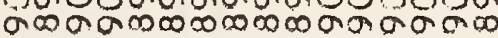

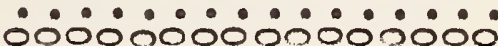

MOOOH-OMmNNNmOCVm

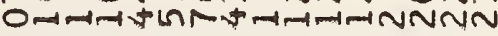

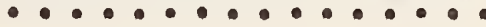
0000000000000000

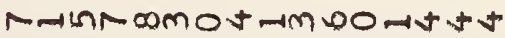
$\bullet \bullet \bullet \bullet \bullet \cdot \bullet \cdot \bullet \cdot$

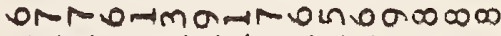

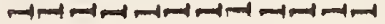

$\frac{1}{x-3}$

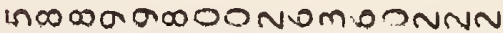

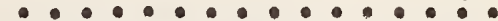

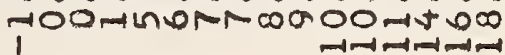

$\frac{z}{d}$ -nMt $\rightarrow-1-1-1+1$ 
ن

$\bigcup_{4 \rightarrow}$

$4=$ w

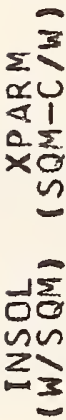

4

42

$a-$

$\sum_{3=1}^{0}$

$-\cdots \cdot \cdot \cdot \cdot \cdot \cdot \cdot \cdot \cdot$ Htmonmino.000 mo.00m Lno Inam-1.00-1-ONMO OR OR-

$\ln$

$0>$

00

zx

NOD DON NANCONNACOND $\bullet \cdot \bullet \cdot \bullet \cdot \bullet \cdot$ $N M=M-N N N N-N N N-N-1$

NNmOLIOA TMLIMNONOS mmmmm तm mmtmmom mmmмलmm 000000000000000

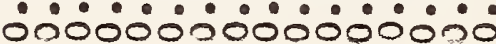

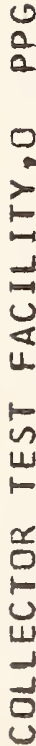

$\sum$

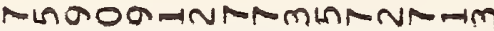

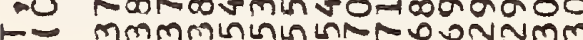

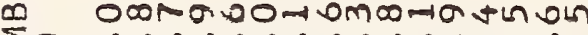

I- .............

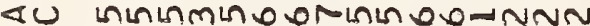

- H-NNNNNNDHATNNNN

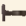

แ०

20

04

10

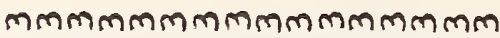

000000000000000 0.000000000000000

$\varangle \rightarrow$

$-\infty$

แU

IO

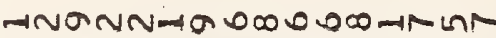

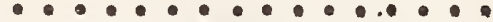

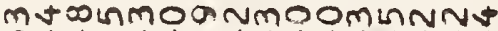

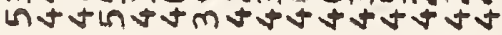

$\sum_{\alpha}$

-NMt $\sum$

wa

$\alpha x$

에

$-$

$>$

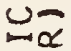

$\Perp \mathrm{C}$

40

w-

$>$

Ua

는

Un

แ-

$>$

U- M oLAminmmmtmmoxo

जII UI novolarknoNaONA-

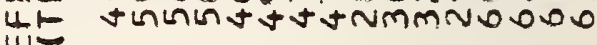
$>$

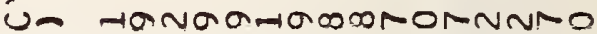
ma $4 \times$ NNOMOOHONMNNOHO-

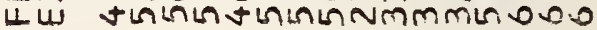

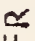

0000000000000000

नaranaranarana

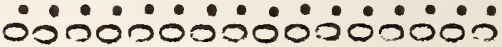

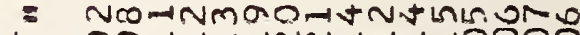

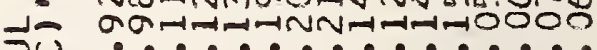

1 เाนก้00000000000000

$\sum^{2}$

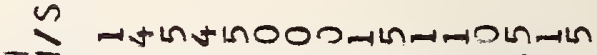

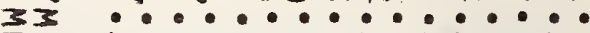

$1=$ taNONOODENTEONTN

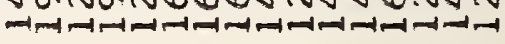

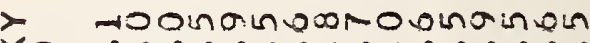

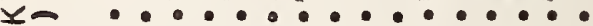
NU MNNA-ANTNNMOIRAR

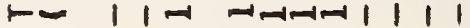

$\underset{2}{\alpha}$ 
$>$

U⿻一⿻冂口十

u. แ.
8303000000009000 .............. 00000000000000000

0000000000000000 - $\cdot \bullet \cdot . \cdot . \cdot$ OHANOODONmNMVNN MMP

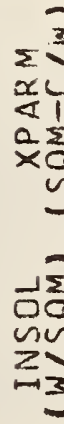

- Do00000000000000 L 2.0 .0

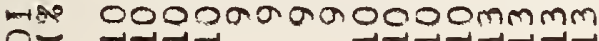

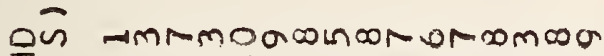
Z1.!.!.!.?

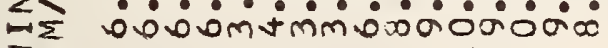
$3=$

辂

MALOS+OHORMLRMOR -1-1-1-1-1-1-1-4-1-1-1-1-1-1-1-

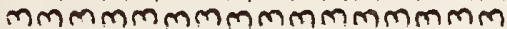
00000000000000 $\cdot \cdot \cdot \cdot \cdot \cdot \cdot \cdot \cdot \cdot \cdot \cdot \cdot \cdot \cdot \cdot$ 0000000000000000

a

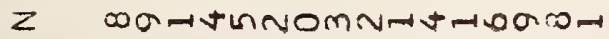

I-

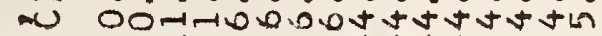
- NVNNDDDONA

or

$>$

$\sum$

$<\omega$

0000000000000000 $\cdot \cdot \cdot \cdot \cdot \cdot \cdot \cdot \cdot \cdot \cdot \cdot \cdot \cdot$ mNmmagagoo-1-mmmm -1-1-1-1-1-1NNNNNNNO

世二

20

Q41

जo

$00000000000000 ?$

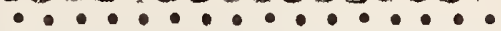
Inininto00000000000 t+t + mmMmmmm VNNN

$<3$

$-\infty$

닌

ID

$\pm 0$

$\sum_{\alpha}^{z}$

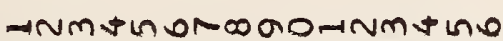

- OAmR tmonmoncon-OOM

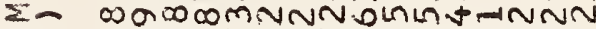

ra Dopom-MNNNNNO000

ax 0050000000000000

all $\cdot \circ \cdot \circ \cdot \circ \cdot \circ \cdot \circ \cdot \circ$ 0000000000000000

$>$

WO MNRMMOONNM

mo

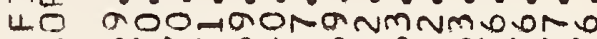

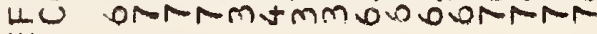

$>$

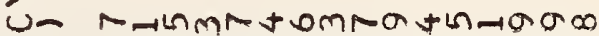

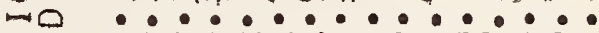

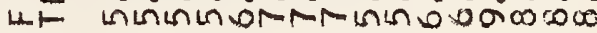

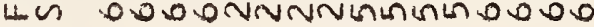

$>$

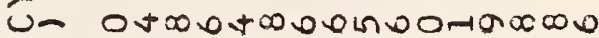

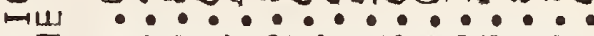

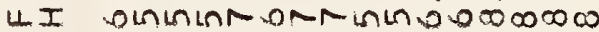

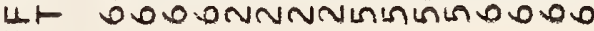

Ü 0000000000000000

mo $4 \times$ OHANOONONMNMDONO

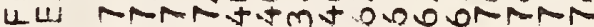

cr

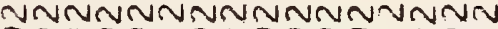
रूजa ن

$=N 00000-m-1000-1-1-N$ ل1- $\infty 0000+0$ in คำำ: inthing 000000000000

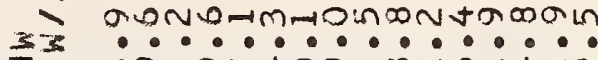
I - Damantoomonantro

$=N N m N-N O N \rightarrow m m+t+t+m$

- Otoolnkningammaver

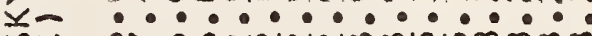

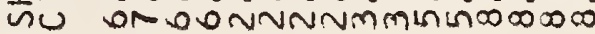
$-1111$

$\underset{\not}{\not}$ -nMth 
$>$

U一

48

4

$\leq$

$\propto \cup$

41

$\frac{2}{x} \frac{1}{3}$

( )

aic
vin
$2=1$

4

$4=$

$0-$

on

$Z \mathbf{Z}$

$3-$

$z$
2
$\alpha$
11
$\frac{1}{2}$
$\frac{1}{2}$

Din
gu

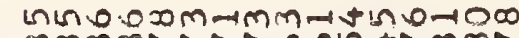

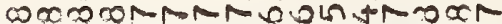

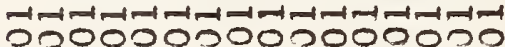
. D00000000000000

$\geq$

$\rightarrow$

$\vdash$

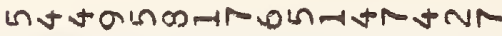
NNNMLIกเกLก

Lit

$>$

$\sum$

\&i)

$\rightarrow$ 1

U⿻

Q⿻日土

QU

$\ln _{n-2}$

oaramannwatatata -. $\cdot \cdot \cdot \cdot \cdot \cdot \cdot \cdot \cdot \cdot$ $t+t+\infty-\infty x-10-1 m m m t$

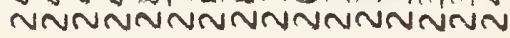

0000000000000000

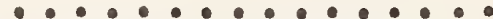
0000000000000000

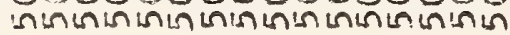

$8-$

$-\infty$

แU⿺

ID

t tanNomw .. *...? orninotmmt tm+oนnmm -1न-1- -1म-1म

$\frac{z}{a}$
$\Sigma-$

$x a$

a Lil

$>$

$\omega$

$\rightarrow \infty$

45

4i)

$>$

U一

$\mapsto 0$

Uా

un

$>$

U-

네

$4 I$

$u=$

$>$

$\bigcup_{i \rightarrow \infty}$

40

Uய

w-

$\propto$

4

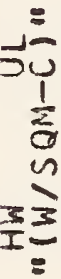

$\underset{x}{\sin }$

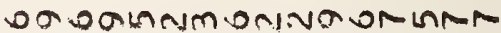
0.? ? ? ? ? ? a joon

-1 $1-1-1-1-1-1$

$\stackrel{z}{a}$

क M M

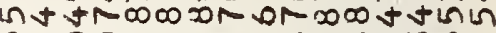
OODONNNNONOOOLIIIL ono0000J0000

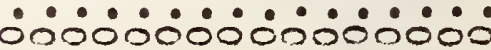

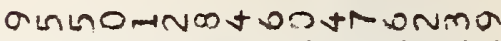
$\cdot \cdot \cdot \cdot \cdot \cdot \cdot \cdot \cdot \cdot \cdot \cdot$ +tinR tmNINNNDOROMO

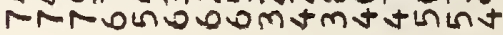

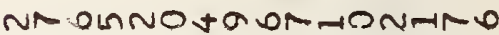
- . . . *...

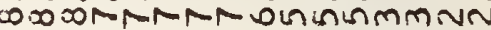

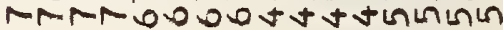

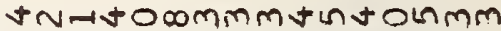
... . . . . . .

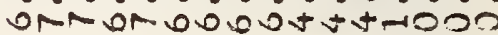
MAMN0000t+tงthinin

MmmmNNNN-1-10-1-1arararararajara

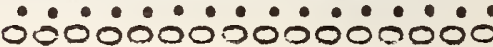

O ODMM

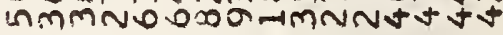

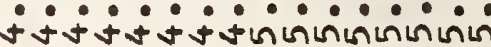

moOR⿻omomrtmintra m. *......... mo0000mLN Ot TONAO

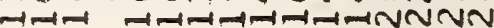

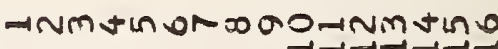


2

Wu 0000000000000000

I 00000000000000

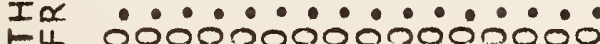

U1.

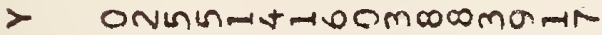

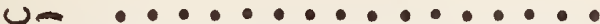

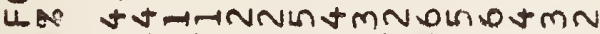

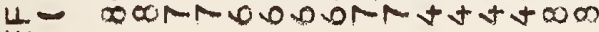

I $\infty$ ormmowntomnow-

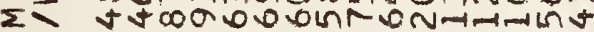

qu OONIVAtJTNNMMAROO

4 1 000000000000000

a $\Sigma$

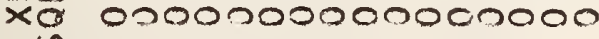

-

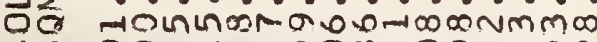

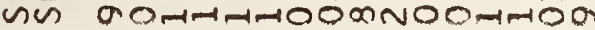

Z 0000000000000000

$\pm$

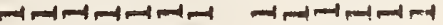

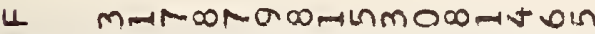

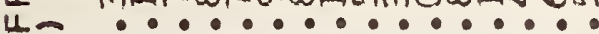

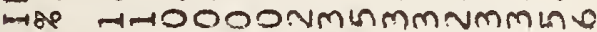

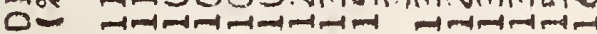

Qus

Nm-inasagainam-10in

$\geq 2$

V. $x=$

0

- ar-h-Nmostingobinto

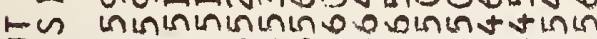
D- $\mathrm{nmmmmmmmmmmmmmmm}$ Do 000000000000000? Ix

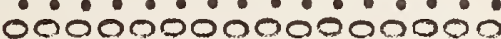

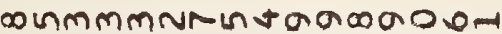

I-

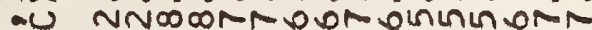

- mminumpringogomm intinf

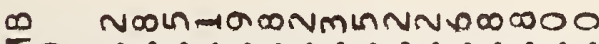

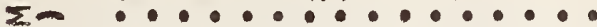

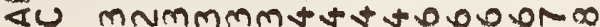
- $\mathrm{mmmmmmmmmmmmmm}$ 1

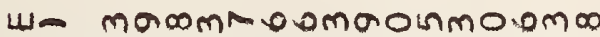
Q

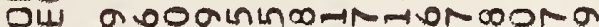
10 mmmNNNmt tIN NN

4 n

4- 00000000000000 七0 wu 0000000000000000

IO

$1-$

$\sum_{\alpha}^{2}$

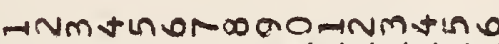

$\Sigma-$

$x a$

$4 x$

a.

$-$

$>$

$0=$

$\rightarrow \alpha$

40

닌

2

0

40

Un

H-

$>$

ज्ञ

U. I

U.

แU

$>$

U.

$4 a$

uᄂ

H-

$\frac{x}{1}$

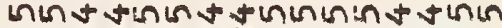

$\frac{1}{20}$
$\frac{1}{0}$
$=3$
$=$

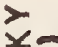

जu

$1 \infty$

$\frac{z}{\alpha}$

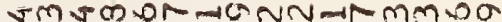

OOt TNNNAmNT000000

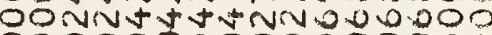
D000000000000000 $\cdot \cdot \cdot \cdot \cdot \cdot \cdot \cdot \cdot \cdot \cdot \cdot \cdot$ 0000000000000000

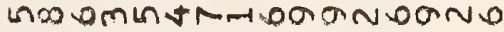

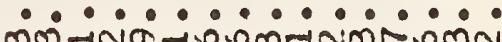
cocor- Ln $000 \mathrm{Mr}+t+t+\infty$

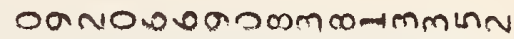

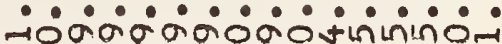
$\infty 000$ inininvom $t+t+\infty 00$

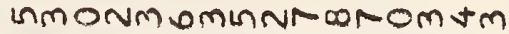
-.........

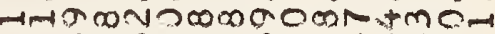
$\infty \infty 0$ ON

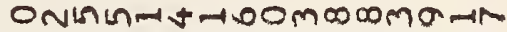
...........

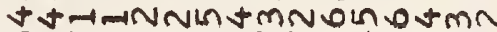

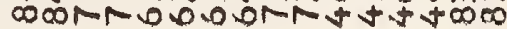

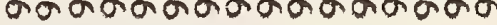
ல0ं0்

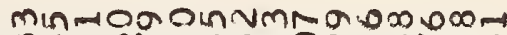

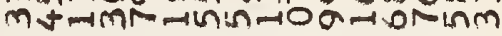

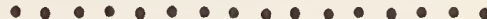
ษ

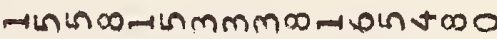
- *......... th mant t NaORGNO $\rightarrow=N$ HNNNON HAMNH

DmmMrom m $+M$ m Nos $-1-1+\infty$ $\bullet \cdot \bullet \cdot \cdot \cdot \cdots \cdot \cdot \cdot \cdot$ NNMNMm * + + NAD NNNNNNNNNNNNNNNN

minmth om 


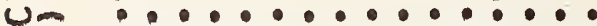

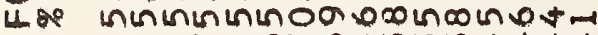
L- MPMDOMDininininst

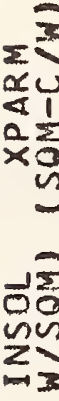

H-1 100 औी $\infty \infty 000$ - In-

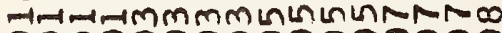
0000000000000000

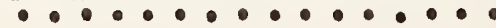
0000000000000000

U.

oิ

$\sum_{i=1}^{2}$

$=$

$\frac{z}{d}$

$I$

$>$

$+$

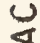

4

แ⿴囗十

Q 0

口us

जo

43

10

ID

-

$\frac{z}{\alpha}$

Oin

2

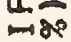

$0-$

ONNIITAR ON00000NM

-........... 00 -

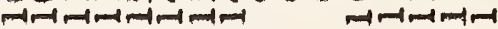

$\bullet \cdot \bullet \cdot \bullet \cdot \bullet \cdot \bullet \cdot \bullet \cdot \bullet$

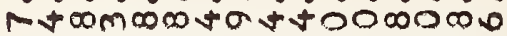

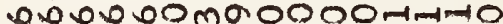

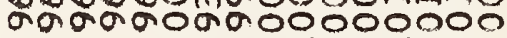

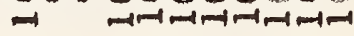

momø OMMm-ATHROLDO

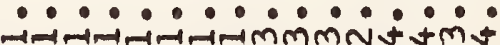

$-i$

$*+ \pm+\infty, 000$ an

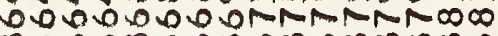
mmmmmmmmmmmmmmm O0000000000000? í0i0: $0.0-10.0-1 m n \cos +\infty+m \varphi$ $\bullet \cdot \bullet \cdot \bullet \cdot \bullet \cdot \bullet \cdot 1$ ninionmmánóninioso - mmmmttt+6010000\%

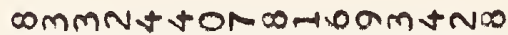

? Nmmtt

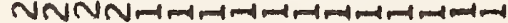

0000000000000000 . . . . ........ 0000000000000000 A tatatatantanatatat

0000000000000200 $\cdot \cdot \cdot \cdot \cdot \cdot \cdot \cdot \cdot \cdot \cdot \cdot \cdot \cdot$ 000000000000000.0

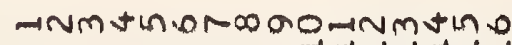

$\Sigma-$

$\alpha 2$

\&x

au

$>$

$0=$

$-\alpha$

4 ?

40

Uు

2

30

$-0$

Hu

II)

2

0

$4 I$

U.m

Li-

3

$m a$

$4 x$

는

H-

$\alpha$

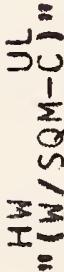

극

us

$1-$

$\frac{z}{\alpha}$

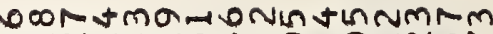
NNNNANO0-10-10NNIN

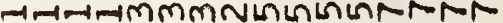
000000000000000 • 0000000000000000

OHONAT DINOINNNOOON

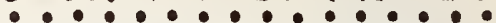

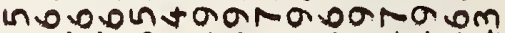
MRM-Novouninintat

OOON-10MmOtat $-100-1$ ค่นกำง

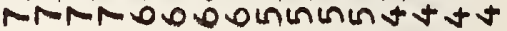

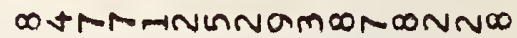
- $\cdot \cdot \cdot \cdot \cdot \cdot \cdot \cdot \cdot \cdot$ $t+t+t h 000 m+m+0$ oON MMNAD000 ininingtam

+HNRNH-10LA-100NNH

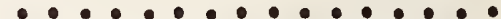

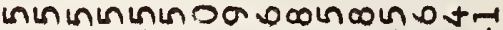

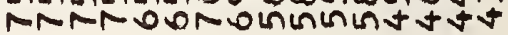

mmmmmmmmmmmmNNNN anararadararara

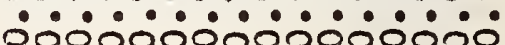

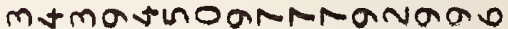
in DINHCONDOMmMNRA ON

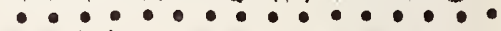

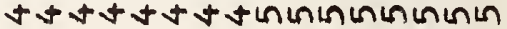

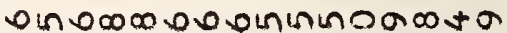
. SNO H. DOONNDONOO

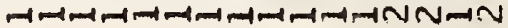

a. $0.0000-\infty 00$ Na - $\cdot \circ \cdot \cdot \cdot \cdot \cdot \cdot \dot{0} \cdot \dot{0}$

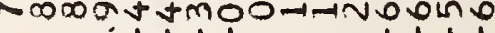

$$
\begin{array}{lll}
1 & 1 & 1 \\
0 & 1 & 1
\end{array}
$$

-nMtin 
$>$

Uu 0000000000000000

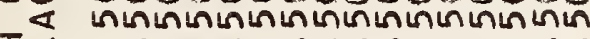
IU

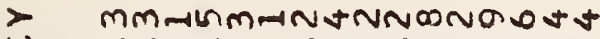

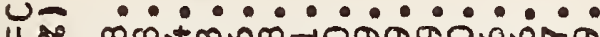

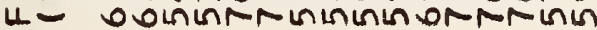

w

- 00000000000000

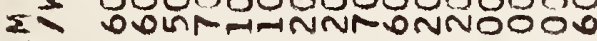

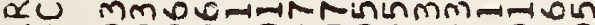
\& 1 00000000000000

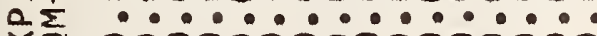
$\times 00000000000000$

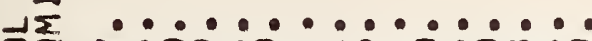

DO NMOO.00न-1Mन1000000 un tint-0.0000m+000 - $00 \mathrm{~m}$

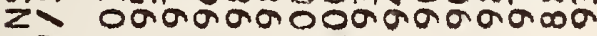

I

L HAGLRARIMMNGSNAmt

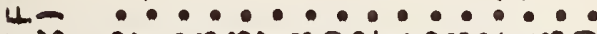

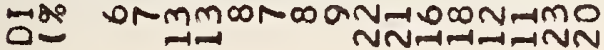

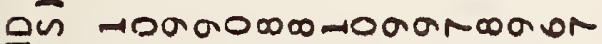

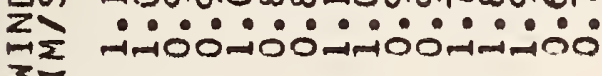

- t+ttmmmmmmmmmmmm tos ininininininininininininininin D- $\mathrm{mmmmmmmmmmmmmmm}$ Do 000000000000000 Ex - 0000000000000000

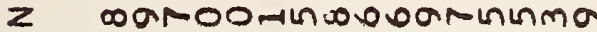
- Ininosommolarts NNR

D INDOTNNARLOHONON

I- . . . . . . . . .

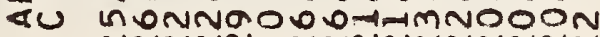
min NNNNNMNNNNNNNNNN $\vdash$

H-

00

ou

我

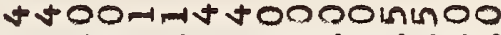
. . . . . . . . . Mmmmmatatatatatat

4

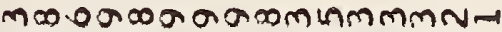
- . . . . .

แU

뜨으

$F$

$\sum_{2}$

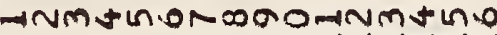

$\Sigma-$

$\operatorname{cac}$

\&.

$-$

$>$

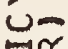

$\rightarrow \infty$

40

แบ

$>$

U.

40

un

$4=$

$>$

บ-

$-4$

$\frac{4}{4}$

$\bar{\omega}_{0}$

$\operatorname{ma}$

$4 \times$

U.tu

u-

$\alpha$

$u$

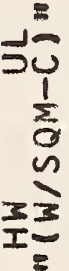

2

जo

10

$\sum_{\alpha}^{z}$

里

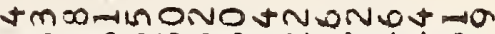
-im-min

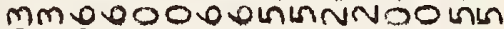
0000000000000000

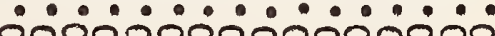

$m \rightarrow-1+10-19 m O N O N+0.0$ $\bullet \bullet \cdot \bullet \cdot \bullet \cdot \bullet \cdot \bullet \cdot$ NM ovininRtatingR

$00-1 m+\infty+1 n m+-4 \cos N 0$

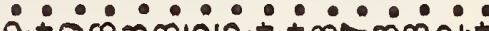
O०

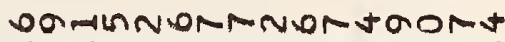
$\bullet \cdot \cdots \cdot \bullet \cdot \bullet \cdot \cdot \cdot$

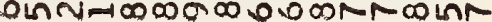

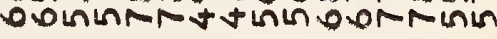

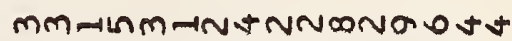
$\bullet \bullet \cdot \bullet \cdot \bullet \cdot \bullet \cdot \cdot \cdot$

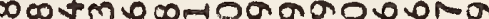

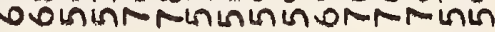

mmmm +

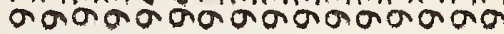

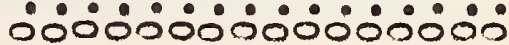

$-\infty$ thm

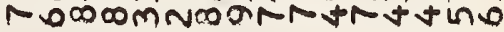
0.0 .0 .0 .0 .0 .0

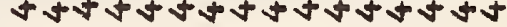

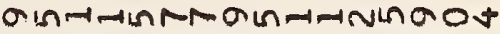
- . . . . aragacosarañNos

mind

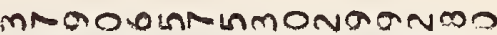

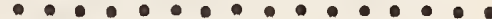
NNMDN DNMO anom tr $\rightarrow \rightarrow-1$

-nMt 


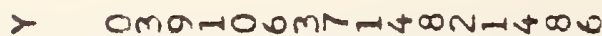
u- ..............

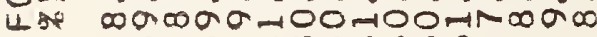
H- 0000N-

UI

12

nin

$\sum_{i=1}$

42

$\rightarrow 20$

0

$\sum_{z=}$

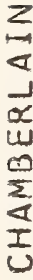

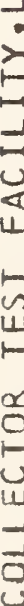

OtwamarionONDINANO

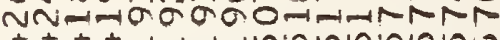
ttat-17

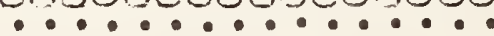
0000000000000000

. ........ ODINNNOHMT-INMNLIR?

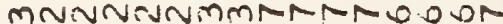

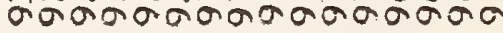

conNon

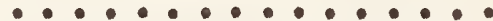
MAON-10m0 -I-INNNN-1-1-1-1-NN

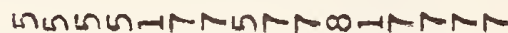
$\bullet \cdot \bullet \cdot \cdot \cdot \cdot \cdot \cdot \cdot \cdot \cdot \cdot \cdot \cdot$ o000mónoo-1000\%

$-\bar{n}$

$0 \rightarrow$

DO

$\Sigma \geq$

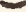

$Z$

10

$1-$

tm+totmranmms $\cdot \bullet \cdot \bullet \cdot \cdot \cdot \cdot \cdot \cdot \cdot \cdot$

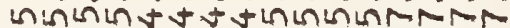
0000 Tath

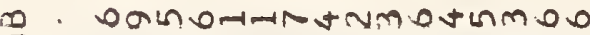

I- . . . . . . . . Lu gabontoogamonthin - NNMMMMmMNNNNMmmm -

$4=$

AO

○以

10

000000000200000 -......

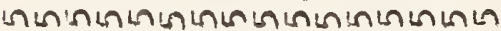

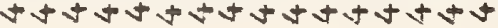

$4-$

$-3$

แ山

I

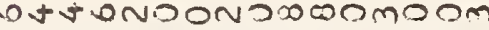

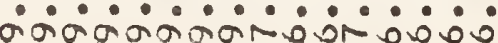

$\frac{z}{\alpha}$

-nmt
INNTR OMESOMONOANm

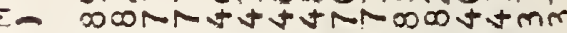

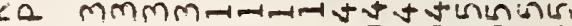

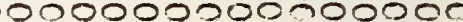

$. \cdot . \cdot \cdot \cdot \cdot \cdot \cdot \cdot \cdot \cdot \cdot$

0000000000000000

$>$

L-

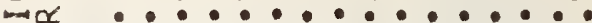

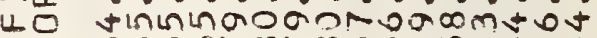

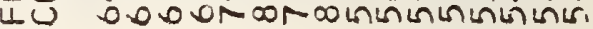

U一

$>$

U- $90 m m-N-D m-N D+*+\infty$

mo ..............

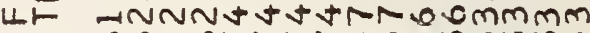

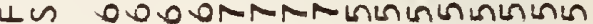
w-

U-

- T

11

$\frac{1}{4}=$

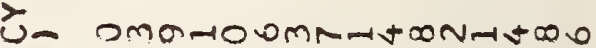
ma $\bullet . \cdot \cdot \cdot \cdot \cdot \cdot \cdot \cdot \cdot \cdot \cdot \cdot$

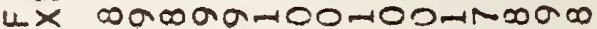

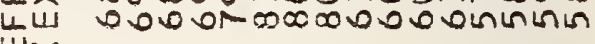

111 -

$\alpha$

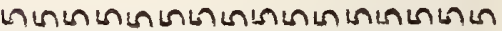
arajagararagara

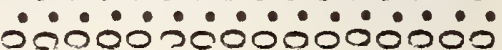

3

ttmmNGOO-HODNMNA N- ITATRNMR ODON.0000

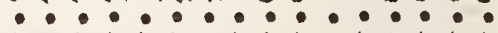

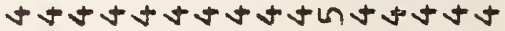

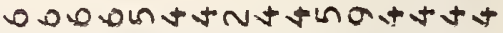
.

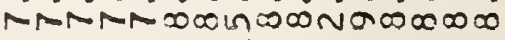
$\rightarrow \rightarrow$

$>$

$x-$

no

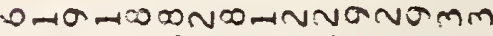

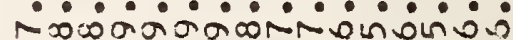

$-1-1-1-1-1-1-1+1-1$ N N NO

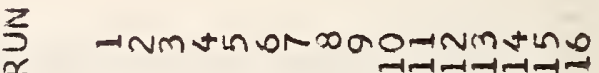


$>$

Wu 2000000000000000 $\varangle 0000000000000000$

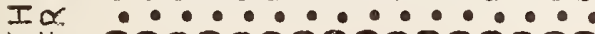
FIL 0000000000000000

$>$

UN

$4-$

世

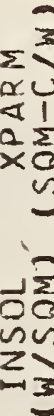

4

$\prod_{-1}$

อ?

$\sum_{3}^{2}$

to

Q)

Do

$\sum \pm$

-

$\geq$

$\lim _{-\infty}$

$1-0$

(1)

$\sum$

$<0$

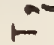

แ-

20

Dய

$\vec{n} 0$

$\mathbb{1 0}$

-O

ID

FD

$\stackrel{z}{a}$

$\infty \infty+00000000$ onv-10

$\cdot \bullet \cdot \bullet \cdot \cdot \cdot \cdot \cdot \cdot \cdot$ GOODNOCONMTMNNLT DO

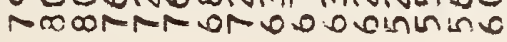

0000000000000000 t t +o NONLIO-MM NONO 000ONNNmmmmmLnL t t 000000000000000 $\cdot \cdot \cdot \cdot \cdot \cdot \cdot \cdot \cdot \cdot \cdot \cdot \cdot \cdot \cdot$ 0000000000000000

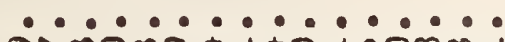
armoma tato-100xक्त

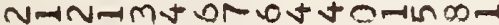
जa

0000000000000000 $\cdot \cdot \cdot \cdot \cdot \cdot \cdot \cdot \cdot \cdot \cdot \cdot$

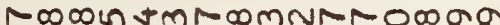

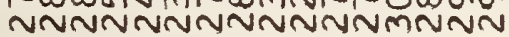

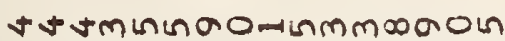

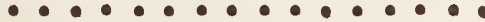
-1-10000-1000-100-1

HIRINNOOMHNLINDOOOO NNNOt

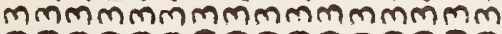
00000000000000

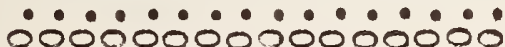
- n-1 otman-moomro

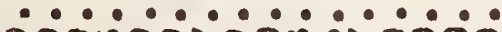
arainaramomóno

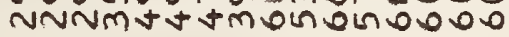

mmmomttotiun otoun

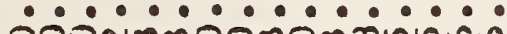
oojnmmoomomounin. mMmmmmmmmmMN vNN

0000000000000000 ..............

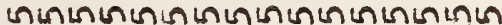

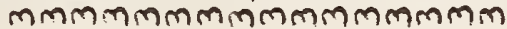

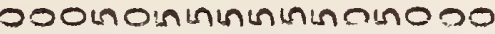

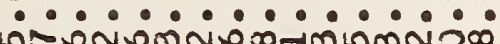
NNN NOMNON-1

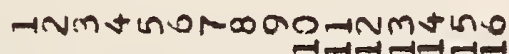

z-

$<a$

$a x$

几山

$-$

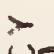

$\bigcup_{\infty}$

40

40

H-

$>$

U.

mD

다

us

$>$

3

UI I

4

ur

3

$\operatorname{ma}$

$4 \times$

나는

$\frac{\alpha}{u}$

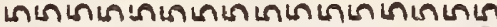
anaranarararara

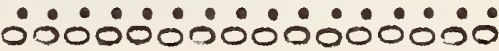

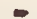

5

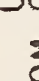

$\frac{1}{8}$

3

$I=$

$y$

जo

$-$

2

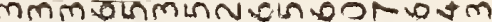

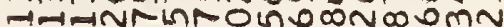

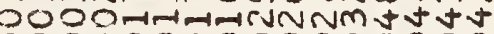
0000000000000000 $\cdot \cdot \cdot \cdot \cdot \cdot \cdot \cdot \cdot \cdot \cdot \cdot \cdot$ 0000000000000000

OMMOG-10-ONtOLMMN

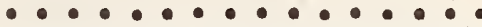

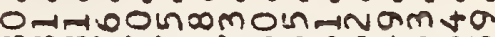

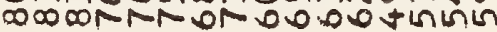

mmm OND $0 N-1-1 m m L N \infty$ - - -

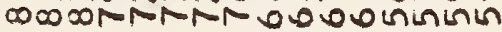

UNATOMROORANGONOW -

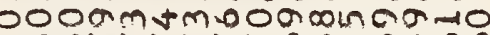

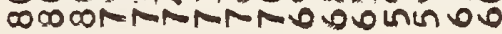

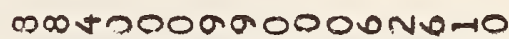
-

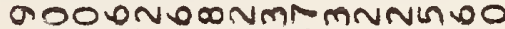

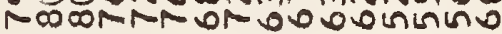

tmanNmR-Atmomgart NNN-Imm tNM thin 000

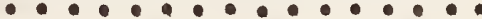

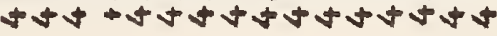

$000000-1$-10000R-1 .............

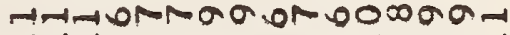

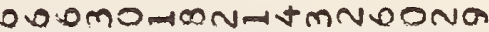
............ conos vmmoommamo-1minN $-1-1 N N N_{-1}-1 \times-1 N-1-1-1$

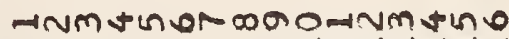
-1म-1माल 
$>$ mormmonminONODOR-

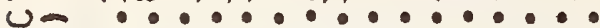

4 of tamadooonanenanmin

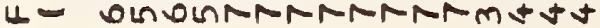

แ1

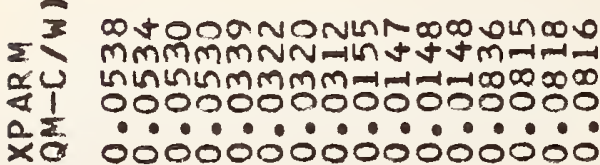

$-\sum_{0}$

un

$\sum_{i=1}^{\infty}$

- *............. talñtand oommolnmtao 0000000000000000

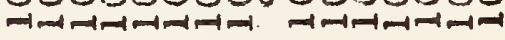

4.

L-

are

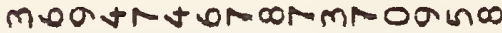

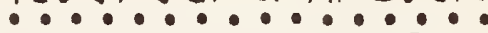

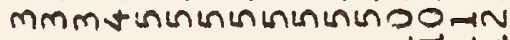

on

DONROONOARTONONm

Z $\pm=$

2

ren

$0>$

00

$\Sigma x$

HtNNtGGanMmmLnNA

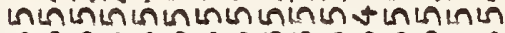

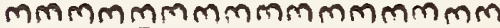
00000000000000

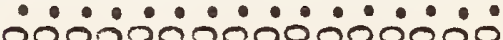

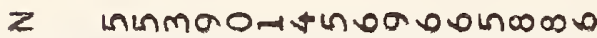

- - ...........

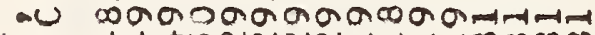

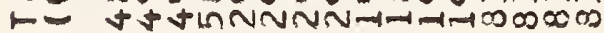

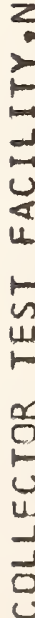

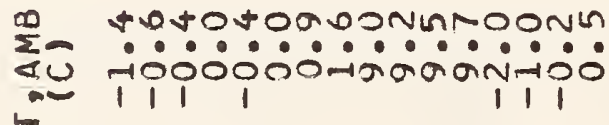
$\leftarrow$

WI 000000000000000

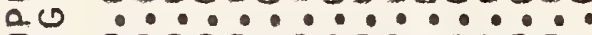
Qu $\vec{n}=$ 0.0000000000000 .00

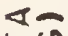

10

1uU

IO

r.

-DOMNASRNMMNRA+N

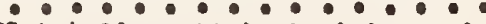
$\infty++\infty N m m N R M M N R M m R$

$\frac{z}{x}$
$\Sigma-$

$\alpha 0$

$\varangle x$

Q.

-

$>$

$\bigcup_{\infty} \propto$

$\stackrel{-1}{\alpha}$

40 แ-

$>$

U一

$\rightarrow 0$

Ut

4 는

$>$

$3-$

-1

$U I$

UF

$>$

$\bigcup_{0}$

$\rightarrow 2$

प4

แ-

$\alpha$

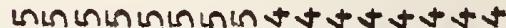
arááááááááa

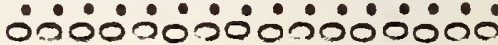

1
50
$\frac{1}{2}$
5
13
15

$>$

는

nை

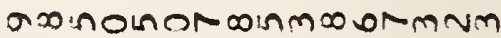
$\cdot \cdot \cdot \cdot \cdot \cdot \cdot \cdot \cdot \cdot \cdot$

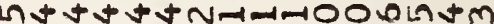
NNNNNNNN-1-A-ANNN

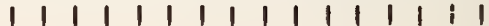

$\sum_{\alpha}^{z}$

MAONMT 0 DON $0000-10-10$

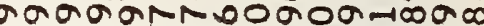

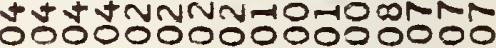

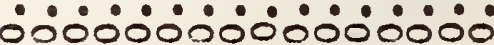

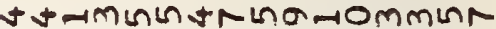

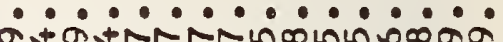

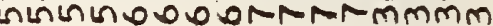

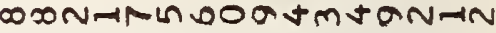
กั Hกน

MNO-In-AtoOHAm tamN $\bullet \cdot \bullet \cdot \bullet \cdot \bullet \cdot \cdot \cdot \cdot \bullet \cdot$ O-n-100000000 NMN mogmmonminONO⿻上Ntamagooolnalninarimin

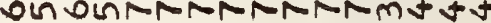

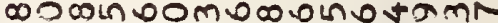
tt t thtmm $00000-1000$ $\cdot \cdot \cdot \cdot \cdot \cdot \cdot \cdot \cdot \cdot \cdot \cdot \cdot$

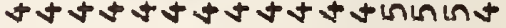

MOR - : * : - * :

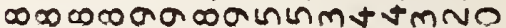
NNNN-1-1-1-1

$-1 N m+40$ onom 
$>$

U-

40

11

U1.

$\sum_{0}^{3}$
0
0
0

0

non

$\sum_{i=1}$

$\pm$

4

$\prod_{\rightarrow \infty}$

0

on

$z_{i=1}$

7

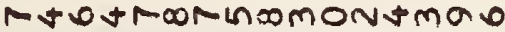

- .........

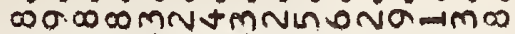

-minominominimio oommntmotrantolom 0000000000000000

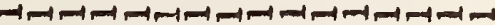

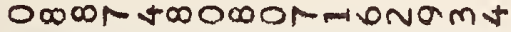
- . . . . In-MNNDRNCONCORORM

N-10-1manomottmoan

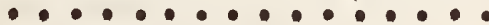
Nmmm-OOH-1 thing

DoOmamox trunammt orthoinsolnt $t+m-1 \mathrm{Nm}-1$

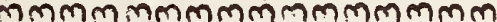
0000000000000000

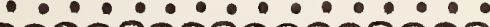
0000000000000000

- NNmmammin oromtomo

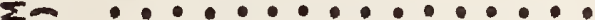

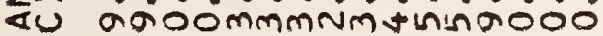
$\rightarrow$ 1 $\rightarrow-1,-1-1-1$

แ-

Q

○ाu

$\rightarrow 0$

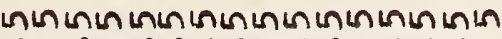
. *.........

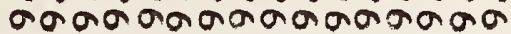
nin

$<\infty$

10

ID

F-

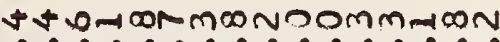

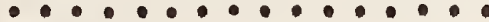

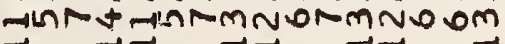

$\sum_{\alpha}^{z}$

-INm
$\Sigma=$

$\propto \alpha$

Qu

$-$

$>$

$\omega \infty$

$\max$

U.

4

w1

$>$

U-

$\rightarrow 0$

uiv

$>$

$\bigcup_{-14}$

4

는

II

$>$

$\bigcup_{\infty \rightarrow \infty}$

$\longrightarrow$ 兘

4

$4=$

$\frac{\alpha}{4}$

mmmmmmmmmNNNMNNN anaragarararara

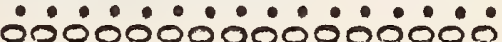

$\frac{2}{20}$
$\frac{1}{2}$
$\frac{1}{2}$
$\frac{3}{8}$

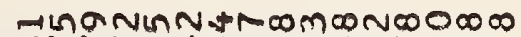
OPRTHA -............ ง

-1ด เกต - . . . . . .

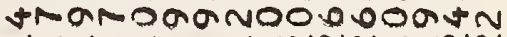

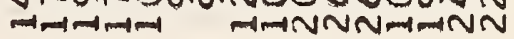

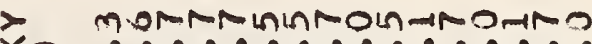

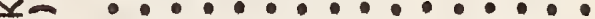

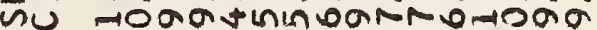
$1-$

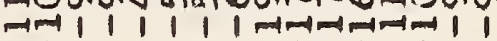

1 I

111111

$\sum_{\alpha}$

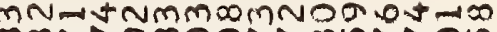

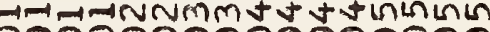
1.0 .000 MORLISOMCOMNMOGOLON $\infty$ Matar DathoNOMN 00000 Dothinothoun

mman NNGHAmasthun

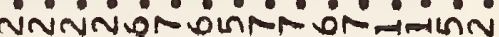

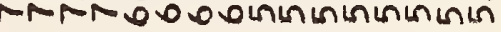

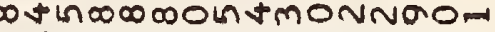

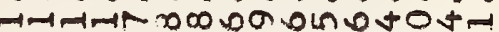

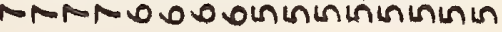

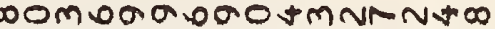
-

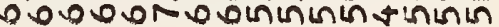

$\rightarrow$ Nmt 
$>$ TNONOOMMONDAMENM

U.

U一

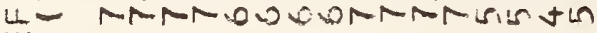

Ui!

$=$

omagoanomoramonm E MANTNMNAJMmNMnN

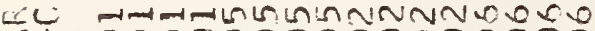
I 1 0040000.000 2:

$\simeq$

- Ot

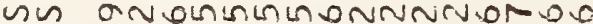
z a000000000002000

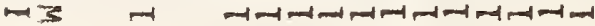

L $0000 \mathrm{mmmm} 00000000$ H.

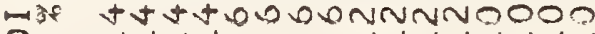

D-

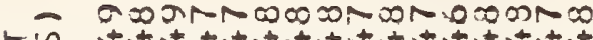

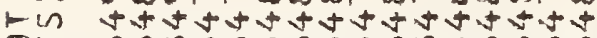

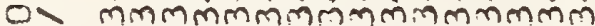
Do 00000000000000 I

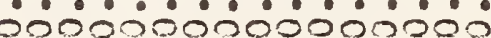

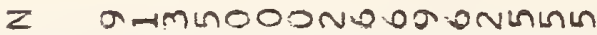

$\sum_{1}$ -U $-\infty \infty \infty-1-1-1000$ -

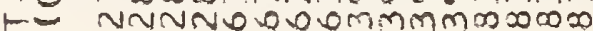

- -hininm:omomoms

$\sum-$

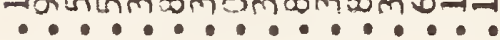

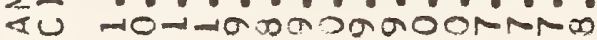

an

NNNN

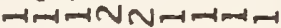

H-

e.

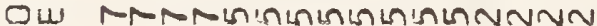

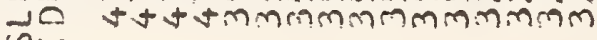

ñ

$\pi-1$

iᄂui

I0

1

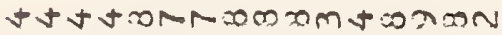
-............ ntin

$\frac{z}{\alpha}$

$>$

$\bigcup_{0}$

UF

Lis แU

$>$

U一

$\rightarrow$ U.

11

45

$>$

U一

10

4

Uiv

$\propto$

30
$\frac{1}{2}$
0
$5=$
$3=$

$\frac{x}{n j}$

$\sum_{a}^{z}$
ront-1R-m motntNRmm

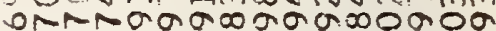

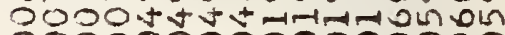
(

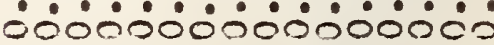

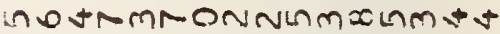

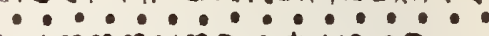

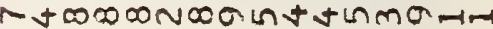

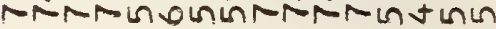

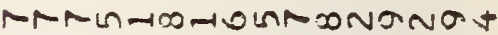

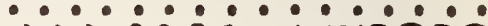

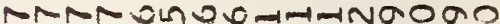

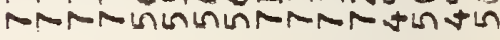

oM-100000 ............

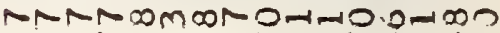

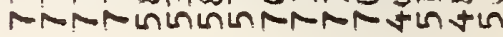

+ NONOAMMONCOtmolom ............

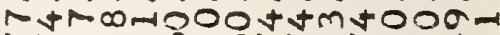

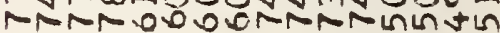

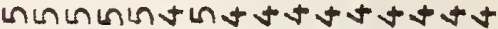

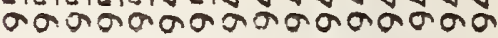

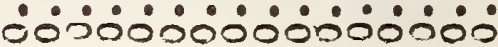

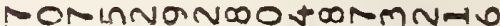
os $x$ co 00 -

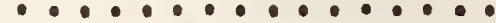

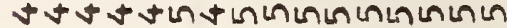

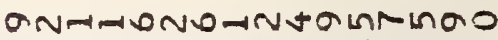
- *...........

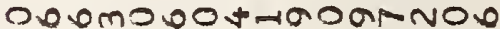
NNMM-INA-AM-NMN-INA

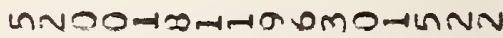
. ............

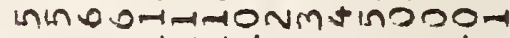
$-1+1-1+1$ 1

1111

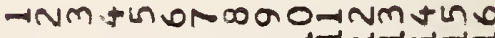


$>$

Wu 0000000000000000

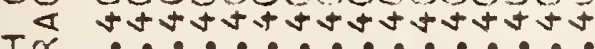

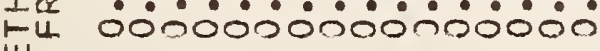
w

$>$

U.

1 of

แU.

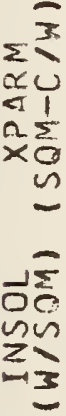

4

4

$\lim ^{-1} x$

$0-$

in

$z_{i=1}$

3

tin

0

20

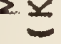

$\sum_{1}$

$\rightarrow \infty$

$1-\infty$

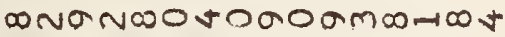

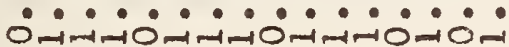

- * * * * *.*.

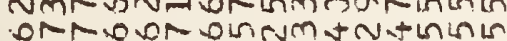
002000000000000? $\rightarrow-1-1-1-1-1-1-1-1-1-1-1-1$

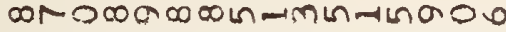

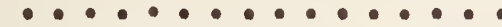
oonagarammñogax -1 $-1-1$

$+1-1-1=$

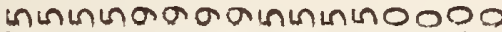
NANR 0.00000000000

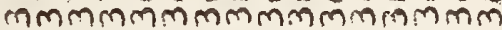
0000000000000000

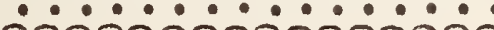
0000000000000000

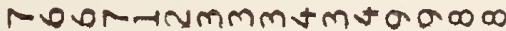
..........

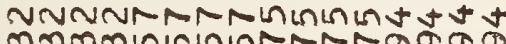

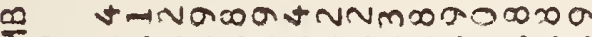

vorion

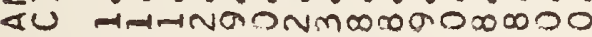

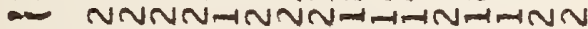
$\vdash$

แล

a)

OW

10

D020000000000000

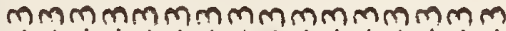
Aatatatatatitati

$\mathbb{1 0}$

닌

I步

$1-$

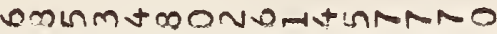
............

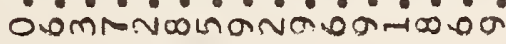

$\sum_{\alpha}^{z}$

-iNm thor $\infty$ anonmtin
20
$a x$
0.11

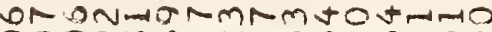
0000inm vNininmm -1m-dOmmmminininin-N-A 0005000000000200 icócicióióióió

$>$

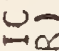

40

40

11]

$>$

$\omega$

-10

un

w-

3.

$\omega$

$m$

4.2

ULt:

U一

$-10$

$4 \times$

4. (L)

w-

$\frac{\sim}{4}$

$t+t+a+t+t+m m \pm m+n$

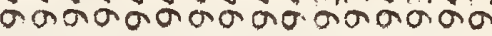

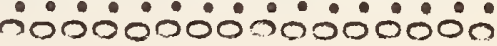

5
50
$\frac{1}{2}$
0
5
$3=$
$=$

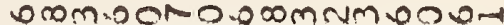
$m+0+00001-\infty-1000 \mathrm{~m}-1$ . AAtatatatahatahth

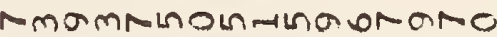
............

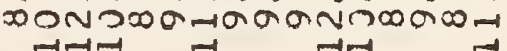

- aindonominatingnaroa

×- ........... ư inininem:

$1-$

$\frac{2}{\alpha}$

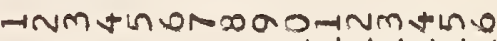


Analytical Relationships Used in the Collector

Model for Analyzing the Effect of Environmental Conditions

NOMENCLATURE

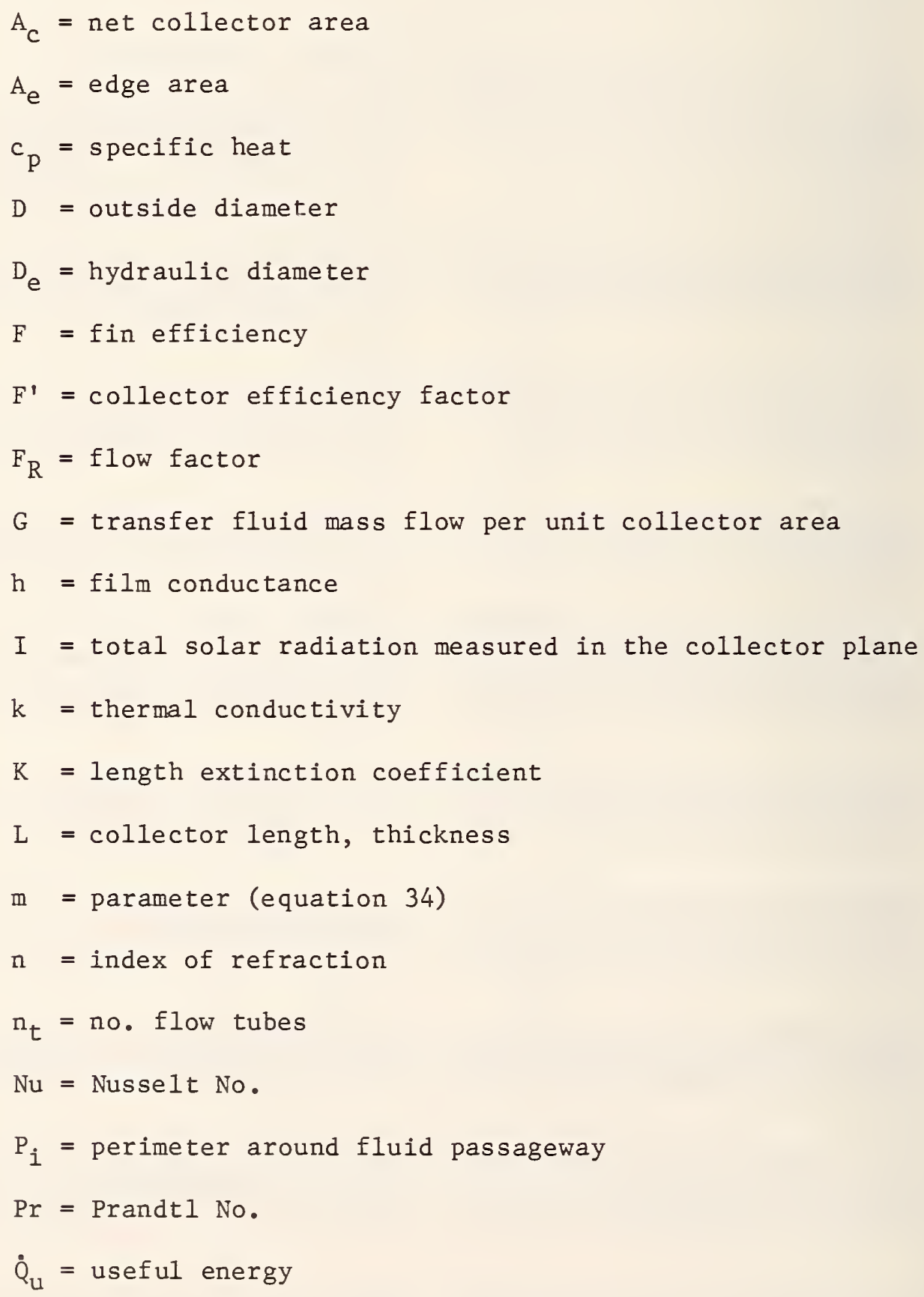




$$
\begin{aligned}
\mathrm{Ra} & =\text { Raleigh No. } \\
\mathrm{Re} & =\text { Reynolds No., based on } \mathrm{D}_{\mathrm{e}} \\
\mathrm{T} & =\text { temperature } \\
\mathrm{U} & =\text { thermal conductance } \\
\mathrm{V}_{\mathrm{W}} & =\text { wind speed } \\
\mathrm{W} & =\text { distance between flow tubes } \\
\alpha & =\text { absorptance } \\
\varepsilon & =\text { emittance } \\
\eta & =\text { efficiency, diffuse fraction } \\
\theta_{2} & =\text { angle between surface normal and beam radiation } \\
\rho & =\text { reflectance } \\
\sigma & =\text { Stefan-Boltzmann Constant } \\
\tau & =\text { transmittance }
\end{aligned}
$$

Subscripts
a air, ambient
b back side, beam
c, c1, c2 cover, no. 1 = inside
ca cover to ambient
d diffuse
e effective, edge
f transfer fluid
fi fluid inlet
i long wavelength diffuse, back insulation
j cover no.
L overall loss 
- fluid-to-ambient loss

p absorber plate

pc plate-to-cover 1

m mean

r radiation

s solar wavelength specular property, sky

$\mathrm{T}$ top loss

a considering absorptance only

12 cover 1 to cover 2

II,I two components of polarization

The collector efficiency is given by

$$
\eta=\frac{\dot{Q}_{u}}{I A_{c}} \times 100 \%
$$

where I is the total solar radiation on the (outer) surface of the cover as measured by a pyranometer in the same plane. The useful heat transfer is given by

$$
\dot{\mathrm{Q}}_{\mathrm{u}}=\mathrm{A}_{\mathrm{c}} \mathrm{F}_{\mathrm{R}}\left((\tau \alpha)_{\mathrm{e}} \mathrm{I}-\mathrm{U}_{\mathrm{L}}\left(\mathrm{T}_{\mathrm{fi}}-\mathrm{T}_{\mathrm{a}}\right)\right)
$$

A summary of the relationships which lead to expressions for $U_{L},(\tau \alpha)$ and $\mathrm{F}_{\mathrm{R}}$ follow. The developments are essentially from reference [6]. Reference [13] was used in developing the expressions for optical properties. Heat transfer correlations for internal flow and natural convection are from references [19] and [20] respectively. Expressions for the properties of air were taken from reference [21].

The expressions are listed for analyzing the two-cover collector. A brief discussion of the changes required to analyze the single cover system follows the list of equations.

The expression for the loss coefficient is developed from

$$
U_{b}=\frac{k_{i}}{L_{i}}
$$




$$
\begin{aligned}
\mathrm{U}_{\mathrm{e}} & =\frac{\mathrm{k}_{\mathrm{e}}}{\mathrm{L}_{\mathrm{e}}} \frac{\mathrm{A}_{\mathrm{e}}}{\mathrm{A}_{\mathrm{c}}} \\
\mathrm{h}_{\mathrm{w}} & =\left\{5.7+3.8\left[\mathrm{~V}_{\mathrm{W}}, \mathrm{m} / \mathrm{s}\right]\right\} \mathrm{W} /\left(\mathrm{m}^{2}{ }^{\circ} \mathrm{C}\right) \\
\mathrm{T}_{\mathrm{s}} & =\left[0.0552\left(\mathrm{~T}_{\mathrm{a}} /{ }^{\circ} \mathrm{K}\right)^{1.5}\right]{ }^{\circ} \mathrm{K} \\
\mathrm{h}_{\mathrm{rpc}} & =\frac{\sigma\left[\mathrm{T}_{\mathrm{pm}}^{2}+\mathrm{T}_{\mathrm{c} 1}^{2}\right]\left[\mathrm{T}_{\mathrm{pm}}+\mathrm{T}_{\mathrm{c} 1}\right]}{1 / \varepsilon_{\mathrm{p}}+1 / \varepsilon_{\mathrm{c} 1}-1} \\
\mathrm{~h}_{\mathrm{pc}} & =\mathrm{Nu}(\mathrm{Ra})\left[\mathrm{k}_{\mathrm{a}} / \mathrm{L}_{\mathrm{pc}}\right] \\
\mathrm{U}_{\mathrm{pc}} & =\mathrm{h}_{\mathrm{rpc}}+\mathrm{h}_{\mathrm{pc}} \\
\mathrm{T}_{\mathrm{c} 2} & =\mathrm{T}_{\mathrm{pm}}-\mathrm{U}_{\mathrm{T}}\left[1 / \mathrm{U}_{\mathrm{pc}}+1 / \mathrm{U}_{12}\right]\left[\mathrm{T}_{\mathrm{pm}}-\mathrm{T}_{\mathrm{a}}\right] \\
\mathrm{T}_{\mathrm{c} 1} & =\mathrm{T}_{\mathrm{pm}}-\left[\mathrm{U}_{\mathrm{T}} / \mathrm{U}_{\mathrm{pc}}\right]\left[\mathrm{T}_{\mathrm{pm}}-\mathrm{T}_{\mathrm{a}}\right] \\
\mathrm{U}_{\mathrm{ca}} & =\mathrm{h}_{\mathrm{w}}+\mathrm{h}_{\mathrm{rca}} \\
\mathrm{h}_{\mathrm{r} 12} & =\frac{\sigma\left[\mathrm{T}_{\mathrm{c} 1}^{2}+\mathrm{T}_{\mathrm{c} 2}^{2}\right]\left[\mathrm{T}_{\mathrm{c} 1}+\mathrm{T}_{\mathrm{c} 2}\right]}{1 / \varepsilon_{\mathrm{c} 1}+1 / \varepsilon_{\mathrm{c} 2}-1} \\
\mathrm{~h}_{12} & =\mathrm{Nu}(\mathrm{Ra})\left[\mathrm{k}_{\mathrm{a}} / \mathrm{L}_{12}\right] \\
\mathrm{U}_{12} & =\mathrm{h}_{\mathrm{r} 12}+\mathrm{h}_{12} \sigma\left[\mathrm{T}_{\mathrm{c} 2}^{4}-\mathrm{T}_{\mathrm{s}}^{4}\right] /\left[\mathrm{T}_{\mathrm{c} 2}-\mathrm{T}_{\mathrm{a}}\right] \\
& =\left[1 / \mathrm{U}_{\mathrm{pc}}+1 / \mathrm{U}_{12}+1 / \mathrm{U}_{\mathrm{ca}}\right]-1 \\
&
\end{aligned}
$$




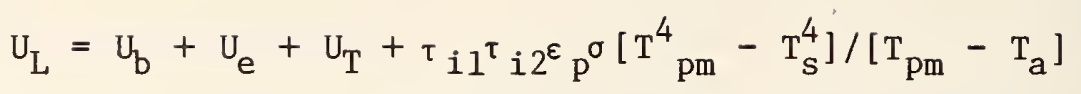

The effective transmittance-absorptance development is

$$
\begin{aligned}
& \theta_{2, j}=\arcsin \left(\sin (\theta) / n_{j}\right) ; j=1,2 \\
& \rho \|, j=\frac{\sin ^{2}\left(\theta_{2}-\theta\right)}{\sin ^{2}\left(\theta_{2}+\theta\right)} \\
& \rho \underline{\underline{I}}, j=\frac{\tan ^{2}\left(\theta_{2}-\theta\right)}{\tan ^{2}\left(\theta_{2}+\theta\right)} \\
& \tau||, j=[1-\rho \|, j]^{2} /\left[1-\pi_{\alpha s}{ }^{2}, j \rho \rho^{2} \mid, j\right] \\
& \left.{ }^{\tau} \underline{\underline{I}, j}=[1-\rho \underline{\underline{1}, j}]^{2 /\left[1-\tau_{\alpha}, j\right.}{ }^{2} \rho \underline{1}, j\right] \\
& \tau_{\alpha s, j}=\exp \left(-L_{c, j} K_{j} / \cos \theta_{2}\right) \\
& \tau_{s, j}=\tau_{a s, j}\left[\tau_{\mid, j}+\tau \mid, j\right] / 2
\end{aligned}
$$

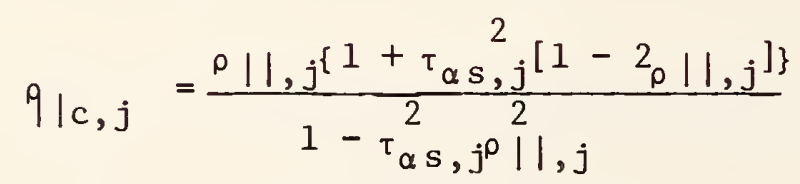

$$
\begin{aligned}
& \rho \perp c, j=\frac{\rho \perp, j\left\{1+\tau_{\alpha s, j}^{2}[1-2 p \perp, j]\right\}}{1-\tau_{\alpha s, j}^{2} q^{2}, j} \\
& \rho_{c, j}=[\rho|| c, j+\rho\lfloor c, j] / 2 \\
& \tau_{s 12}=\tau_{s 1} \tau_{s 2} /\left[1-\rho_{c 1} \rho_{c 2}\right] \\
& \rho_{c 12}=\rho_{c 1}+\rho_{c 2}{ }^{2}{ }_{s 1} /\left[1-\rho_{c 1} \rho_{c 2}\right] \\
& \pi / 2 \\
& \tau_{S d}=\int_{0} \tau_{S}(\theta) \sin (2 \theta) d \theta
\end{aligned}
$$


where $\tau_{s}(\theta)$ is given by equation (25) or (29) for 1 or 2 covers, respectively.

$$
(\tau \alpha)=\frac{\alpha_{p}\left\{\left[1-n_{d}\right] \tau_{s 12}+\eta_{d} \tau_{s d, 12\}}\right.}{1-\left[1-\alpha_{p}\right] \rho_{c 12}\left(60^{\circ}\right)}
$$

Finally,

$$
\begin{aligned}
(\tau \alpha)_{\mathrm{e}}= & (\tau \alpha)+\tau_{s 2}\left[1-\tau_{\alpha s, 1}\right]\left[1-\mathrm{U}_{\mathrm{pa}} / \mathrm{U}_{\mathrm{pc}}\right]+ \\
& {\left[1-\tau_{\alpha s, 2}\right]\left\{1-\mathrm{U}_{\mathrm{pa}}\left[1 / \mathrm{U}_{\mathrm{pc}}+1 / \mathrm{U}_{12}\right]\right\} }
\end{aligned}
$$

The expression for $F_{R}$ is developed from

$$
\begin{aligned}
& m=\left(U_{L} /\left[k_{p} L_{p}\right]\right)^{1 / 2} \\
& F=\sinh (m[W-D] / 2) /\{\cosh (m[W-D] / 2) m[W-D] / 2\} \\
& N_{f}(\operatorname{RePrDe} / L)=3.66+\frac{0.0668\left[\operatorname{RePrD} e^{/ L}\right]}{1+0.04\left(\operatorname{RePrD} D_{e} / L\right)^{2 / 3}} \\
& \left.h_{f}={N u_{f}}_{\mathrm{f}_{f}} / \mathrm{D}_{e}\right] \\
& U_{O}=\left\{W\left[1 /\left\{U_{L}[D+F(W-D)]\right\}+1 /\left[P_{i} h_{f}\right]\right\}^{-1}\right. \\
& \quad F^{\prime}=U_{o} / U_{L} \\
& F_{R}=\left[G c_{p} / U_{L}\right]\left\{1-\exp \left(-F^{\prime} U_{L} /\left[G c_{p}\right]\right)\right\}
\end{aligned}
$$

Since the calculations proceed in an iterative fashion, the following expressions are used to converge the plate and fluid temperatures.

$$
\begin{aligned}
& \mathrm{T}_{\mathrm{fm}}=\mathrm{T}_{\mathrm{fi}}+\dot{Q}_{\mathrm{u}} /\left\{\mathrm{A}_{\mathrm{c}} \mathrm{U}_{\mathrm{L}} \mathrm{F}_{\mathrm{R}}\left[1-\mathrm{F}_{\mathrm{R}} / \mathrm{F}^{\prime}\right]\right\} \\
& \mathrm{T}_{\mathrm{pm}}=\mathrm{T}_{\mathrm{fm}}+\dot{Q}_{\mathrm{u}} \mathrm{P}_{i} \mathrm{~h}_{\mathrm{f}} /\left[\mathrm{Ln}_{\mathrm{t}}\right]
\end{aligned}
$$


For collector No. 2 with a single cover, equations (10-12) and (17) were not used. The subscript "c2" in equation (13) was replaced with "cl", $1 / \mathrm{U}_{12}$ was deleted in equation (15) and $\tau_{i 2}$ was deleted from equation (18). In a similar fashion, $j=1$ in the $(\tau \alpha)$ development; equations (29) and (30) were not used; the 2 's were deleted from the subscripts in equation (32); the last term and $\tau_{s 2}$ were deleted from equation ( 3 ); and, finally, the fin efficiency, equation ( 35 ), was essentially unity.

The order of progression of computation using a digital computer for this model is described. The environmental conditions, operating conditions, collector dimensions, heat transfer properties, and other quantities shown in Table C1 must be specified to calculate efficiency. The computational procedure uses several iterative loops to handle the nonlinear dependence of $\mathrm{U}_{\mathrm{L}}$ on temperature. Equations (3-6) and (19-32) are solved. Equation (31) is integrated numerically using a trapezoidal scheme. For a specified inlet temperature, a trial value several degrees higher is assumed for the mean plate temperature. The cover temperatures are calculated from equations (16) and (17) for the assumed mean plate temperature until the former converge within $0.5^{\circ} \mathrm{C}$. A top-loss coefficient is determined from equation (15) for the trial plate temperature. Equations (18) and (33-35) are next solved. The mean fluid temperature is assumed initially as the plate temperature to determine fluid properties and the convection heat transfer coefficient using equations (36) and (37). The collector efficiency factor and heat removal factor are calculated from equations (38-40). The useful heat transfer is next determined from equation (2) and subsequently used in equation (41) to check the assumed mean fluid temperatures. The calculations are repeated using the updated fluid temperatures until convergence within $0.5^{\circ} \mathrm{C}$ is obtained. The mean plate temperature is then calculated from equation (42) and compared with the assumed value. The calculations starting with equation (16) are repeated until the plate mean temperature converges to within $0.5^{\circ} \mathrm{C}$. Finally, the collector efficiency is determined from equation (1).

The analytical model and computer code were verified by checking final results with a desk calculator and by comparison with other theoretical results in the literature. In particular, the result for the theoretical model agrees with those shown in references [2] and [22].

The required dimensions were taken from the collector manufacturer's literature [23, 24] and from the information in Table 1. The heat transfer properties for the collector materials were generally those provided by the manufacturers or from a handbook [25]. The dimensions and heat transfer properties used for both collectors No. 1 and 2 are shown in Table C1. Since a considerable amount of uncertainty was associated with the effective value of the edge-loss coefficient, it was selected primarily on the basis of giving the best least squares fit of theoretical to experimentally determined efficiencies. The experimental results also influenced the selection of the "extinction coefficient" of the cover materials when used with the index of refraction shown. With these two parameters specified, equations (19-29) can be used to calculate transmittance values in terms of the beam incident angle. 
Table C1. Summary of Environmental and Operating Conditions and Collector Properties for the Theoretical Performance Model

Environmental and Operating Conditions: "Standard" Values

$$
\begin{array}{lll}
\mathrm{T}_{\mathrm{a}}=20^{\circ} \mathrm{C} & \mathrm{U}=1000 \mathrm{~W} / \mathrm{m}^{2} & \mathrm{~V}_{\mathrm{W}}=3 \mathrm{~m} / \mathrm{s} \\
\eta_{\mathrm{d}}=0.15 & \mathrm{G}=0.02 \mathrm{~kg} /\left(\mathrm{s}^{\circ} \mathrm{m}^{2}\right) & \theta=15^{\circ} \\
\mathrm{S}=45^{\circ} & \text { Ethylene Glycol Concentration }=0.0
\end{array}
$$

Dimensions and Heat Transfer Properties

A. PPG Collector; $A_{c}=1.602 \mathrm{~m}^{2}$

$$
\begin{aligned}
& \mathrm{L}=0.841 \mathrm{~m} \\
& \mathrm{n}_{\mathrm{t}}=13 \\
& D \quad=1.27 \mathrm{~cm} \\
& \mathrm{D}_{\mathrm{e}}=1.2 \mathrm{~cm} \\
& \mathrm{P}_{\mathrm{i}}=1.5 \mathrm{~cm} \\
& \mathrm{~L}_{\mathrm{p}}=1.524 \mathrm{~mm} \\
& \mathrm{k}_{\mathrm{p}}=221 \mathrm{~W} /\left(\mathrm{m}^{\circ}{ }^{\circ} \mathrm{C}\right) \\
& \varepsilon_{\mathrm{p}}=0.92 \\
& \alpha_{\mathrm{p}}=0.95 \\
& \mathrm{~L}_{\mathrm{i}}=5.35 \mathrm{~cm} \\
& \mathrm{k}_{\mathrm{i}}=0.045 \mathrm{~W} /\left(\mathrm{m}^{\circ}{ }^{\circ} \mathrm{C}\right) \\
& \mathrm{U}_{\mathrm{e}}=1.50 \mathrm{~W} /\left(\mathrm{m}^{2} \cdot{ }^{\circ} \mathrm{C}\right) \\
& \mathrm{L}_{\mathrm{pc}}=0.953 \mathrm{~cm} \\
& \varepsilon_{c}=0.85 \\
& \tau_{i c}=0.02 \\
& \mathrm{n} \quad=1.518 \\
& \mathrm{KL}_{\mathrm{c}}=0.04 \\
& \mathrm{~L}_{12}=0.953 \mathrm{~cm}
\end{aligned}
$$

B. CMC Collector; $A_{C}=1.789 \mathrm{~m}^{2}$

$$
\begin{aligned}
& \mathrm{L} \quad=0.860 \mathrm{~m} \\
& \mathrm{n}_{\mathrm{t}}=19 \\
& D \quad=4.07 \mathrm{~cm} \\
& \mathrm{D}_{\mathrm{e}}=0.5 \mathrm{~cm} \\
& \mathrm{P}_{\mathrm{i}}=8.5 \mathrm{~cm} \\
& \mathrm{~L}_{\mathrm{p}}=0.911 \mathrm{~mm} \\
& \mathrm{k}_{\mathrm{p}}=47.6 \mathrm{~W} /\left(\mathrm{m}^{\circ}{ }^{\circ} \mathrm{C}\right) \\
& \varepsilon_{\mathrm{p}}=0.12 \\
& \alpha_{p}=0.96 \\
& \mathrm{~L}_{\mathrm{i}}=7.62 \mathrm{~cm} \\
& \mathrm{k}_{\mathrm{i}}=0.045 \mathrm{~W} /\left(\mathrm{m}^{\circ}{ }^{\circ} \mathrm{C}\right) \\
& \mathrm{U}_{\mathrm{e}}=0.90 \mathrm{~W} /\left(\mathrm{m}^{2} \cdot{ }^{\circ} \mathrm{C}\right) \\
& \mathrm{L}_{p c}=1.9 \mathrm{~cm} \\
& \varepsilon \quad=0.88 \\
& \tau_{i c}=0.02 \\
& \mathrm{n} \quad=1.518 \\
& \mathrm{KL}_{c}=0.035
\end{aligned}
$$


NBS.114A (REV. 11-77)

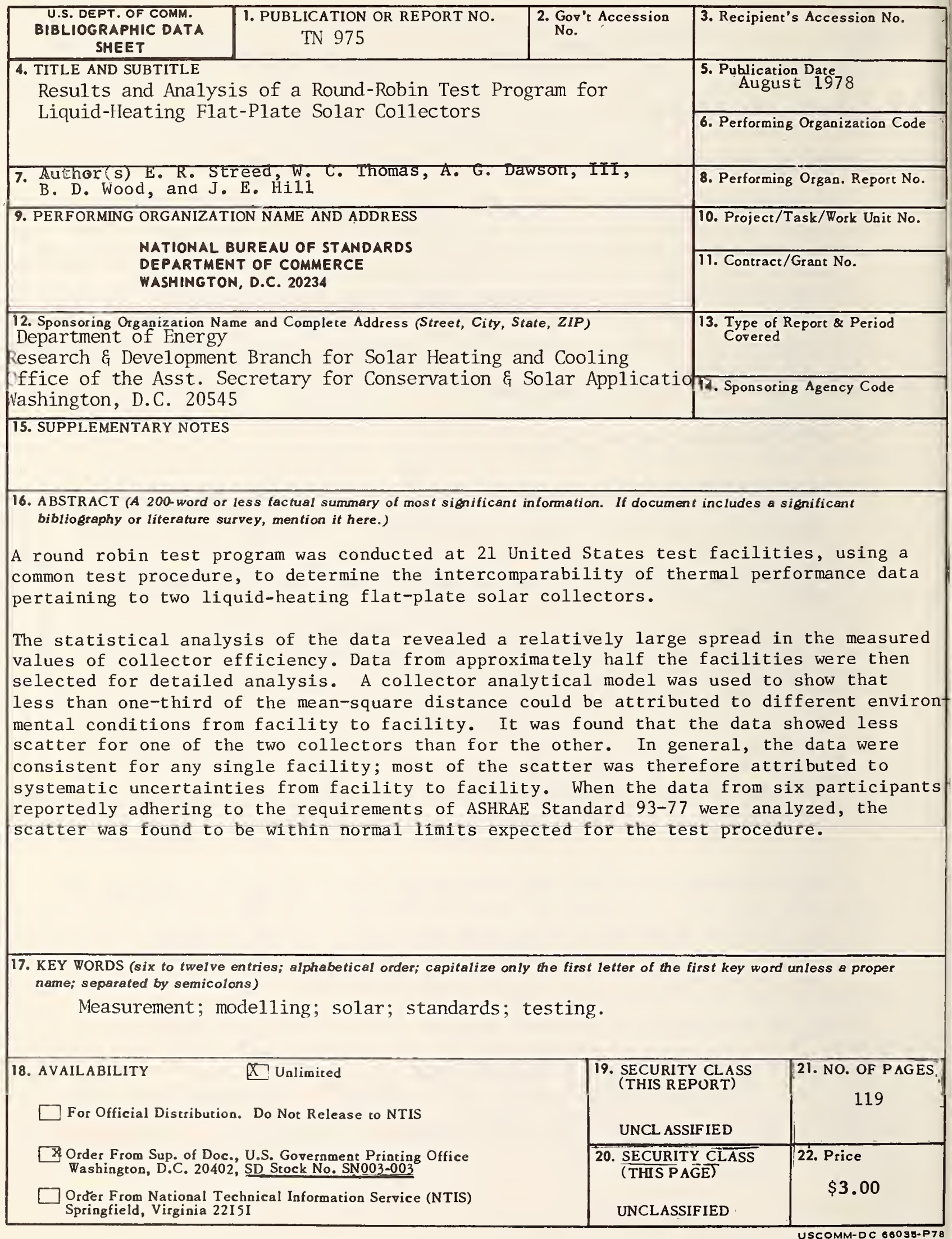




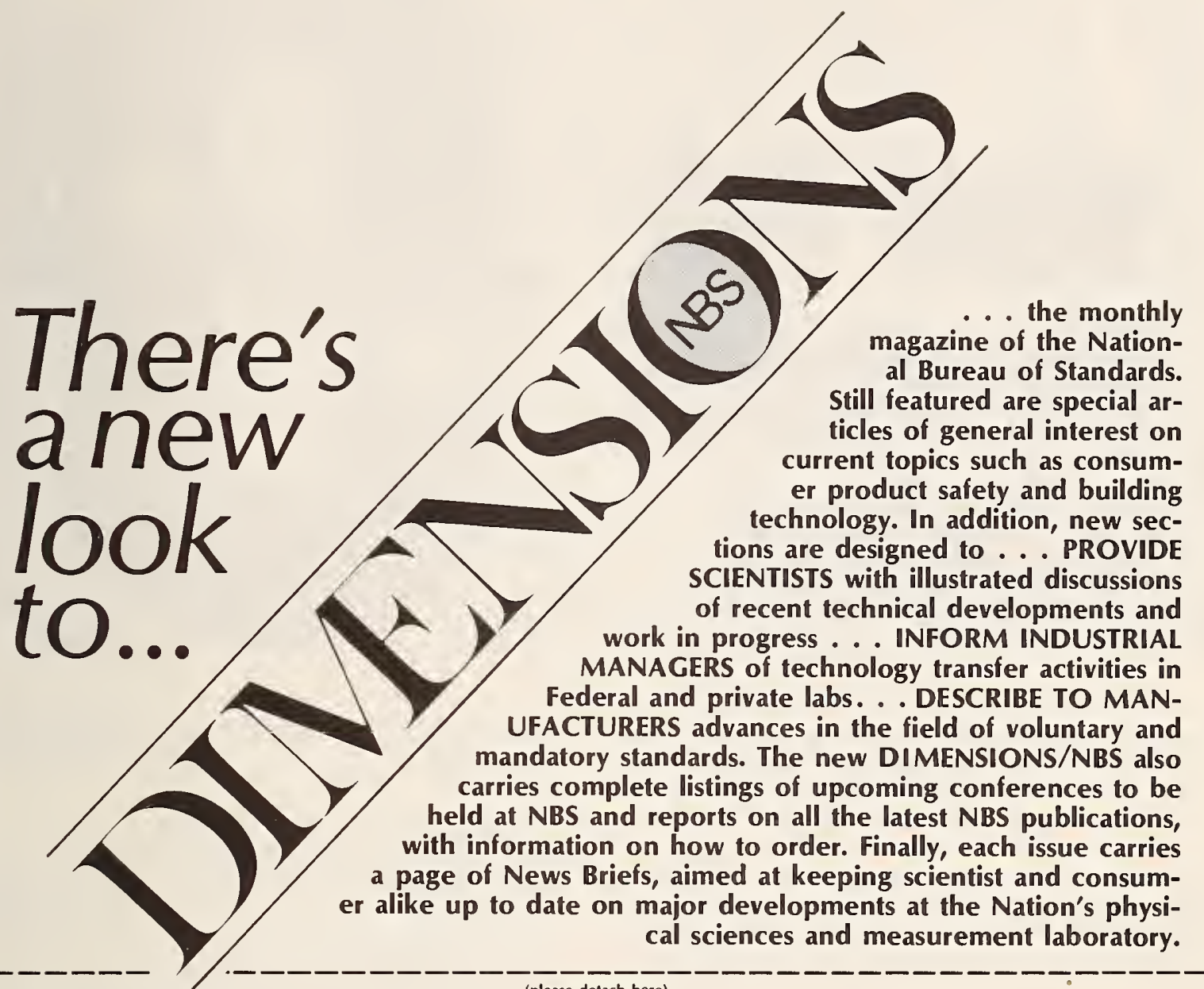

(please detach here)

SUBSCRIPTION ORDER FORM

Enter my Subscription To DIMENSIONS/NBS at $\$ 12.50$. Add $\$ 3.15$ for foreign mailing. No additional postage is required for mailing within the United $\varsigma^{+}$ttes or its possessions. Domestic remittances should be made either by postal money order, express money order, or check. Foreign remittances should be made either by international money order, draft on an American bank, or by UNESCO coupons.

Send Subscription to:

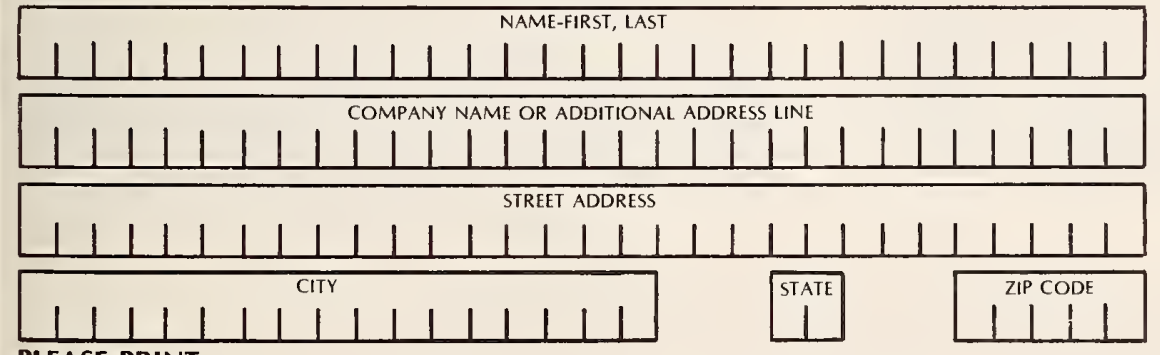

Remittance Enclosed (Make checks payable to Superintendent of Documents)

Charge to my Deposit Account No.

MAIL ORDER FORM TO: Superintendent of Documents Government Printing Office Washington, D.C. 20402

\section{PLEASE PRINT}




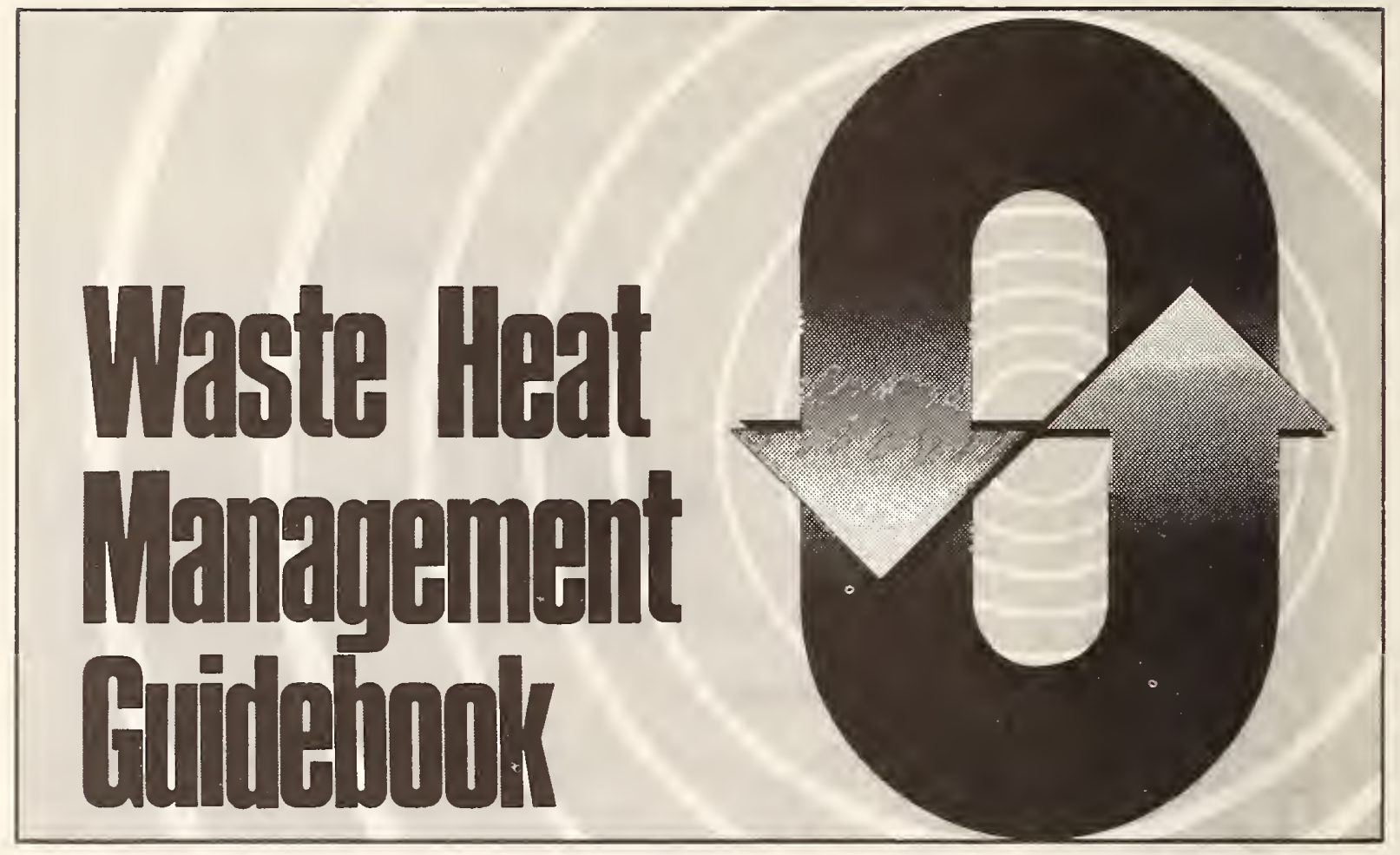

A typical plant can save about 20 percent of its fuel-just by installing waste heat recovery equipment. But with so much equipment on the market, how do you decide what's right for you?

Find the answers to your problems in the Waste Heat Management Guidebook, a new handbook from the Commerce Department's National Bureau of Standards and the Federal Energy Administration.

The Waste Heat Management Guidebook is designed to help you, the cost-conscious engineer or manager, learn how to capture and recycle heat that is normally lost to the environment during industrial and commercial processes.

The heart of the guidebook is 14 case studies of companies that have recently installed waste heat recovery systems and profited. One of these applications may be right for you, but even if it doesn't fit exactly, you'll find helpful approaches to solving many waste heat recovery problems.
In addition to case studies, the guidebook contains information on:

- sources and uses of waste heat

- determining waste heat requirements

- economics of waste heat recovery

- commercial options in waste heat recovery equipment

- instrumentation

- engineering data for waste heat recovery

- assistance for designing and installing waste heat systems

To order your copy of the Waste Heat Management Guidebook, send \$2.75 per copy (check or money order) to Superintendent of Documents, U.S. Government Printing Office, Washington, D.C. 20402. A discount of 25 percent is given on orders of 100 copies or more mailed to one address.

The Waste Heat Management Guidebook is part of the EPIC industrial energy management program aimed at helping industry and commerce adjust to the increased cost and shortage of energy. 


\section{PERIODICALS}

JOURNAL OF RESEARCH-The Journal of Research of the National Bureau of Standards reports NBS research and development in those disciplines of the physical and engineering sciences in which the Bureau is active. These include physics, chemistry, engineering, mathematics, and computer sciences. Papers cover a broad range of subjects, with major emphasis on measurement methodology, and the basic technology underlying standardization. Also included from time to time are survey articles on topics closely related to the Bureau's technical and scientific programs. As a special service to subscribers each issue contains complete citations to all recent NBS publications in NBS and nonNBS media. Issued six times a year. Annual subscription: domestic $\$ 17.00$; foreign $\$ 21.25$. Single copy, $\$ 3.00$ domestic; $\$ 3.75$ foreign.

Note: The Journal was formerly published in two sections: Section A "Physics and Chemistry" and Section B "Mathematical Sciences."

\section{DIMENSIONS/NBS}

This monthly magazine is published to inform scientists, engineers, businessmen, industry, teachers, students, and consumers of the latest advances in science and technology, with primary emphasis on the work at NBS. The magazine highlights and reviews such issues as energy research, fire protection, building technology, metric conversion, pollution abatement, health and safety, and consumer product performance. In addition, it reports the results of Bureau programs in measurement standards and techniques, properties of matter and materials, engineering standards and services, instrumentation, and automatic data processing.

Annual subscription: Domestic, \$12.50; Foreign $\$ 15.65$.

\section{NONPERIODICALS}

Monographs-Major contributions to the technical literature on various subjects related to the Bureau's scientific and technical activities.

Handbooks - Recommended codes of engineering and industrial practice (including safety codes) developed in cooperation with interested industries, professional organizations, and regulatory bodies.

Special Publications-Include proceedings of conferences sponsored by NBS, NBS annual reports, and other special publications appropriate to this grouping such as wall charts, pocket cards, and bibliographies.

Applied Mathematics Series-Mathematical tables, manuals, and studies of special interest to physicists, engineers, chemists, biologists, mathematicians, computer programmers, and others engaged in scientific and technical work.

National Standard Reference Data Series-Provides quantitative data on the physical and chemical properties of materials, compiled from the world's literature and critically evaluated. Developed under a world-wide program coordinated by NBS. Program under authority of National Standard Data Act (Public Law 90-396).
NOTE: At present the principal publication outlet for these data is the Journal of Physical and Chemical Reference Data (JPCRD) published quarterly for NBS by the American Chemical Society (ACS) and the American Institute of Physics (AIP). Subscriptions, reprints, and supplements available from ACS, 1155 Sixteenth St. N.W., Wash., D.C. 20056.

Building Science Series-Disseminates technical information developed at the Bureau on building materials, components, systems, and whole structures. The series presents research results, test methods, and performance criteria related to the structural and environmental functions and the durability and safety characteristics of building elements and systems. Technical Notes-Studies or reports which are complete in themselves but restrictive in their treatment of a subject. Analogous to monographs but not so comprehensive in scope or definitive in treatment of the subject area. Often serve as a vehicle for final reports of work performed at NBS under the sponsorship of other government agencies.

Voluntary Product Standards--Developed under procedures published by the Department of Commerce in Part 10 , Title 15, of the Code of Federal Regulations. The purpose of the standards is to establish nationally recognized requirements for products, and to provide all concerned interests with a basis for common understanding of the characteristics of the products. NBS administers this program as a supplement to the activities of the private sector standardizing organizations.

Consumer Information Series-Practical information, based on NBS research and experience, covering areas of interest to the consumer. Easily understandable language and illustrations provide useful background knowledge for shopping in today's technological marketplace.

Order above NBS publications from: Superintendent of Documents, Government Printing Office, Washington, D.C. 20402.

Order following NBS publications-NBSIR's and FIPS from the National Technical Information Services, Springfield, Va. 22161.

Federal Information Processing Standards Publications (FIPS PUB)-Publications in this series collectively constitute the Federal Information Processing Standards Register. Register serves as the official source of information in the Federal Government regarding standards issued by NBS pursuant to the Federal Property and Administrative Services Act of 1949 as amended, Public Law 89-306 (79 Stat 1127), and as implemented by Executive Order 11717 (38 FR 12315, dated May 11, 1973) and Part 6 of Title 15 CFR (Code of Federal Regulations).

NBS Interagency Reports (NBSIR)-A special series of interim or final reports on work performed by NBS for outside sponsors. (both government and non-government). In general, initial distribution is handled by the sponsor; public distribution is by the National Technical Information Services (Springfield, Va. 22161) in paper copy or microfiche form.

\section{BIBLIOGRAPHIC SUBSCRIPTION SERVICES}

The following current-awareness and literature-survey bibliographies are issued periodically by the Burean:

Cryogenic Data Center Current Awareness Service. A literature survey issued biweekly. Annual subscription: Domestic, $\$ 25.00$; Foreign, $\$ 30.00$.

Liquified Natural Gas. A literature survey issued quarterly. Annual subscription: $\$ 20.00$.
Superconducting Devices and Materials. A literature survey issued quarterly. Annual subscription: $\$ 30.00$. Send subscription orders and remittances for the preceding bibliographic services to National Bureau of Standards, Cryogenic Data Center (275.02) Boulder, Colorado 80302. 
U.S. DEPARTMENT OF COMMERCE

National Bureau of Standards

Washington, D.C. 20234

POSTAGE AND FEES PAID U.S. DEPARTMENT OF COMMEACE COM-2I5

OFFICIAL BUSINESS

Penalty for Private Use, $\$ 300$
SPECLAL FOURTH-CLASS RATE BOOK 





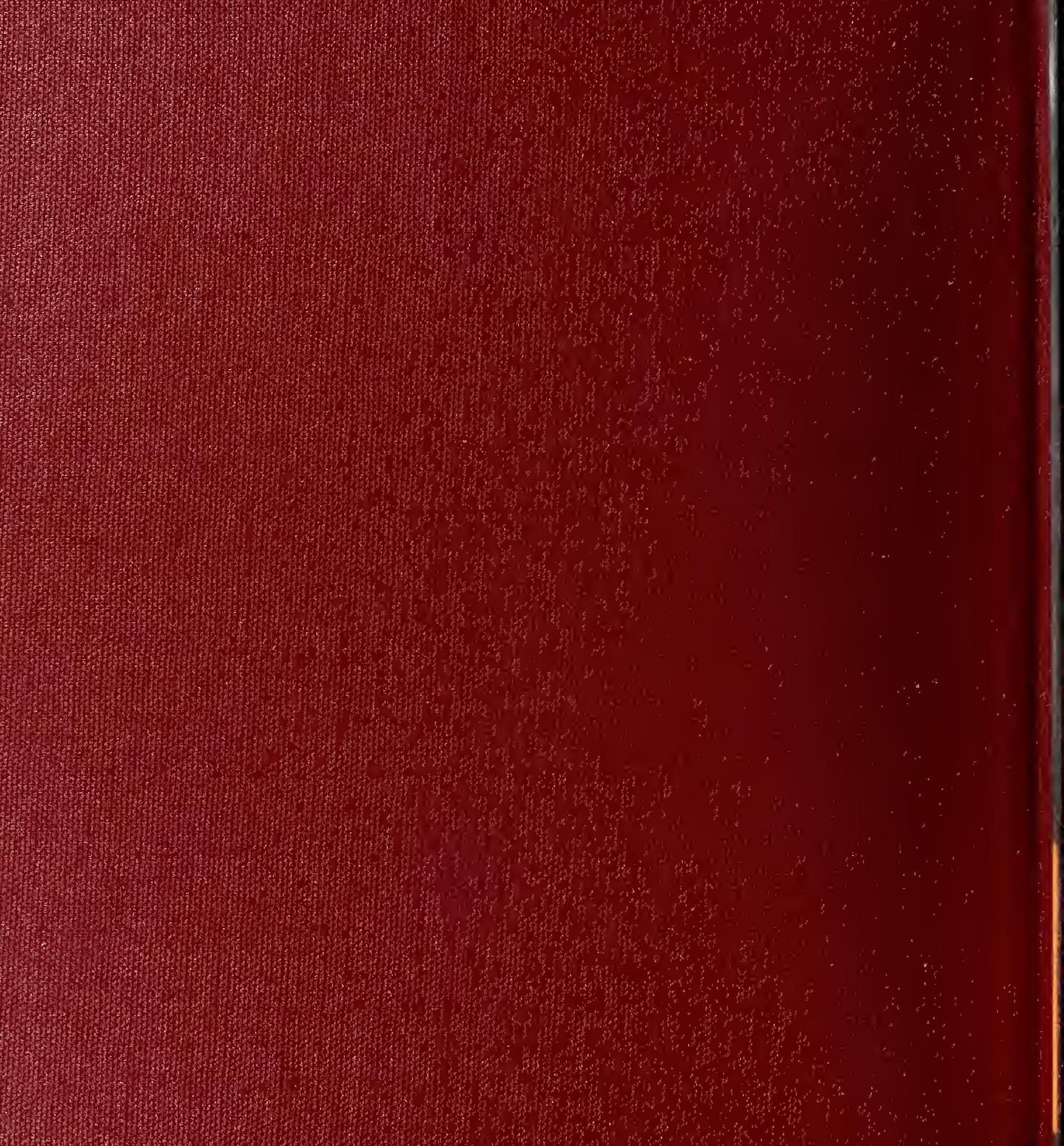

W

7.

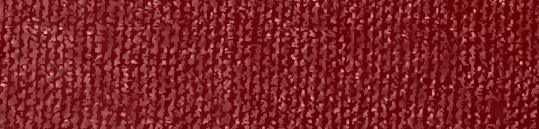

(7)

(3)

H.th 